NBER WORKING PAPER SERIES

\title{
NAÏVE *BUYING* DIVERSIFICATION AND NARROW FRAMING BY INDIVIDUAL INVESTORS
}

\author{
John Gathergood \\ David Hirshleifer \\ David Leake \\ Hiroaki Sakaguchi \\ Neil Stewart \\ Working Paper 25567 \\ http://www.nber.org/papers/w25567 \\ NATIONAL BUREAU OF ECONOMIC RESEARCH \\ 1050 Massachusetts Avenue \\ Cambridge, MA 02138 \\ February 2019, Revised February 2020
}

We thank David Laibson, George Loewenstein and Brigitte Madrian for very helpful comments and suggestions, together with seminar participants at the Network for Integrated Behavioural Science Annual Workshop 2018, University of Glasgow, University of Bristol, University of Loughborough and Queen Mary University of London. This work was supported by Economic and Social Research Council grants ES/K002201/1, ES/N018192/1, ES/P008976/1, and Leverhulme grant RP2012-V-022. Sakaguchi acknowledges PhD studentship funding from the Economic and Social Research Council with award reference 1499648. The views expressed herein are those of the authors and do not necessarily reflect the views of the National Bureau of Economic Research.

NBER working papers are circulated for discussion and comment purposes. They have not been peer-reviewed or been subject to the review by the NBER Board of Directors that accompanies official NBER publications.

(C) 2019 by John Gathergood, David Hirshleifer, David Leake, Hiroaki Sakaguchi, and Neil Stewart. All rights reserved. Short sections of text, not to exceed two paragraphs, may be quoted without explicit permission provided that full credit, including $\odot$ notice, is given to the source. 
Naïve *Buying* Diversification and Narrow Framing by Individual Investors

John Gathergood, David Hirshleifer, David Leake, Hiroaki Sakaguchi, and Neil Stewart

NBER Working Paper No. 25567

February 2019, Revised February 2020

JEL No. D14,D53,D91,G02,G11,G12

\section{ABSTRACT}

We provide the first tests to distinguish whether individual investors equally balance their overall portfolios (naïve portfolio diversification-NPD) or engage in naïve buying diversification (NBD) - equally balancing values in same-day purchases of multiple assets. We find NBD in purchases of multiple stocks, and in mixed purchases of individual stocks and funds. In contrast, there is little evidence of NPD. So investors seem to narrowly frame their buy-day decision. Simulation analysis suggests that NBD substantially reduces investor welfare. These findings suggest that behavioral finance theory should incorporate transactional as well as portfolio framing.

John Gathergood

School of Economics

Sir Clive Granger Building

University Park

Nottingham

NG8 1AA

United Kingdom

john.gathergood@nottingham.ac.uk

David Hirshleifer

The Paul Merage School of Business

University of California, Irvine

4291 Pereira Drive

Irvine, CA 92697

and NBER

david.h@uci.edu

David Leake

Lloyds Banking Group

David.E.Leake@lloydsbanking.com
Hiroaki Sakaguchi

University of Warwick

Department of Psychology

hiro.sakaguchi@outlook.com

Neil Stewart

Warwick Business School

University of Warwick

England

neil.stewart@warwick.ac.uk. 


\section{Introduction}

When faced with financial decisions, people often turn to simple rules to guide their choices. Examples include setting saving to be a constant fraction of income, or choosing a mortgage contract that keeps monthly repayments at a target value. Such heuristics are sometimes very imperfect approximations to optimal behavior, or even quasi-rationally optimal behavior. So, to develop realistic models of individual behavior, and to inform policy design, it is important to understand these heuristics.

We here investigate how investors approach a very fundamental financial choice: how to allocate funds for investment across multiple stocks. Portfolio theory offers standard recommendations for how to diversify optimally across stocks (Markowitz 1952), but individual investors deviate substantially from what theory recommends (Goetzmann and Kumar 2008). ${ }^{1}$

One of the major explanations that have been proposed to explain this failure is narrow framing, which is the tendency to make choices in isolation rather than making an integrated overall decision (Gilovich and Griffin 2010). One proposed consequence of narrow framing is the reluctance of investors to incorporate new assets into their portfolios, providing an explanation for limited stock market participation and portfolio under-diversification (Barberis, Huang, and Thaler 2006; Barberis and Huang 2008).

Our focus is on an aspect of narrow framing that has received much less attention, and on neglect of something even more basic - the benefit of coordinating the amounts of new purchases of an asset with current holding of that same asset in the portfolio. We first show that when investors buy stocks, they often group several trades together on the same day, and split their investments evenly using the $1 / N$ heuristic. This raises the question of whether, when making a purchase, investors seek to bring their overall portfolios into balance, or whether they apply $1 / N$ only to their new purchases. If they frame narrowly upon their new purchases, such a heuristic could potentially push their

\footnotetext{
${ }^{1}$ Investors typically hold only a few stocks and exhibit biases such as over-trading (Barber and Odean 2000), sensitivity to gains compared with losses (Odean 1998) and rank effects (Hartzmark 2015). See also the reviews of behavioral finance of Hirshleifer (2015) and Barberis (2018), and the review of investor behavior of Barber and Odean (2013).
} 
overall holdings further from a $1 / N$ balance. We find that investors do indeed use $1 / N$ as a buying heuristic, not as a portfolio balancing heuristic.

This evidence indicates that investors act very narrowly, naïvely diversifying among what they buy, not what they hold. Investors appear to be considering only the stocks they are buying on a given day. This is consistent with narrow framing at the daily purchase level independently from their existing portfolio. We call this behavior Nä̈ve Buying Diversification (NBD). The main contribution of this paper is to show that investors engage in NBD and do not engage in Naive Portfolio Diversification (NPD) defined as equal weighting of securities in their portfolios. In other words, many investors split their buys according to the $1 / N$ heuristic, but very few investors allocate buy amounts so as to achieve $1 / N$ portfolio shares.

We also explore whether investors' use of NBD is associated with fundamental category errors. Individual stocks and funds are very different categories of investment, so that under rational balancing of risk and return, there is no presumption that investments across these categories, either in new purchases or in overall portfolios, should be at all close to equally weighted. Nevertheless, we find NBD among investors when buying stocks and funds on the same day, even when buying index-tracking Exchange Traded Funds (ETFs) on the same day as individual stocks. This behavior also results in portfolios that are heavily tilted toward a few stocks, which is inconsistent with NPD. Such behavior reflects a fundamental investor confusion. ${ }^{2}$

These findings mark a departure from the approach of both rational models and many existing behavioral finance models. In many psychology-based models of investment choices, even though investors do not behave rationally, they engage in portfolio framingthey seek to optimize an overall portfolio in order to achieve an attractive probability distribution of consumption (Daniel, Hirshleifer, and Subrahmanyam 2001; Grinblatt and Han 2005; Li and Yang 2013; Barberis, Mukherjee, and Wang 2016). Our findings provide a new type of empirical support for the hypothesis that investors narrowly frame on

\footnotetext{
${ }^{2}$ Investors who have private information may have good reasons to choose portfolios heavily tilted toward particular stocks. But this does not provide a sensible reason to tilt heavily toward a stock in order to equalize the amount invested in a stock and a fund on a given buy-day.
} 
incremental gambles (as in Barberis and Huang 2001). We show that the tracks of such narrow framing are evident in the incremental purchases of assets already in the portfolio, and that the resulting $1 / N$ behavior makes it especially clear that investors are engaged in narrow framing on the incremental purchase.

Our findings indicate that investors narrowly frame on incremental portfolios of gambles, not just single ones. In consequence, errors of narrow framing go beyond judging incorrectly how risky it is to acquire the additional gamble, resulting in mistaken decisions over whether to make a new investment (as reflected, for example, in application to the stock market participation puzzle), or how much in total to invest in a new gamble. It also leads to errors in the relative weights investors allocate to the different securities that they buy, conditional on making a purchase. Our finding that investors often diversify with equal weighting within the new portfolio gamble being acquired provides a new kind of test for and confirmation of narrow framing on new gambles. This new kind of test focuses on relative weights within the transaction portfolio rather than on the decision of whether to invest. We refer to the investor's narrow focus on the transaction portfolio as transactional framing.

Our evidence also indicates that investors fall far short of even quasi-rational portfolio optimization. To an extent that leaves tracks in the data, investors do not even seem to be trying to assess security or portfolio risk, nor to diversify or bring balance to their overall portfolios - only to bring value-invested balance to purchase events on a day-by-day basis. Trading based on equalizing trade values rather than estimating risks seems even farther from rational than the narrow framing of investors who, in the above-mentioned models of narrow framing, overestimate the risks of certain potential portfolio changes.

We are not the first to study the use by investors of naïve diversification heuristics, meaning the deliberate use of $1 / N$ value allocations. Our findings differ in two key ways. First, our study resolves a debate in the literature as to whether individuals actually use $1 / N$ as a heuristic for investing. Previous studies provide evidence that employees make $1 / N$ allocations in establishing their regular contribution rates to retirement savings plans 
(Benartzi and Thaler 2001, Huberman and Jiang 2006). The debate centers on whether such $1 / N$ allocations result from a $1 / N$ choice heuristic or arise from a framing effect associated with the number of options, $N$, offered in the menu. Our findings provide evidence that individuals actually do use $1 / N$ as a heuristic, since we examine a general stock investment setting in which there is no plan-ordained number of available assets " $N$ " in the menu of options available to investors.

The second key way in which our findings differ derives from the fact that we examine an empirical setting that permits sharp distinction between NBD and NPD. Based on this, we document that NBD is present whereas NPD is not. In the Benartzi and Thaler (2001) and Huberman and Jiang (2006) retirement saving plan testing context, it is not possible to distinguish between NBD and NPD, because investors choose continuing contribution rates rather than one-time transactions, which very strongly links specific purchase transactions weights to overall portfolio weights. This is especially the case given very strong evidence of inertia in retirement investing (Madrian and Shea 2001). This distinction is important, since findings of naïve diversification from past literature are often described as a bias toward NPD. ${ }^{3}$

In contrast with these studies, our tests focus on a sample of discrete trades by individual investors, so that there is a clear distinction between buy-transaction weights and portfolio weights, and therefore between NBD and NPD. This is one of the key innovations of our study. Our finding that investors use NBD when buying both individual stocks and funds (which are comprised of multiple stocks) on the same day provides especially clear evidence that investors are not using a heuristic of equalizing the weights across stocks in their portfolios.

This evidence raises the question of whether, and how much, NBD damages portfolio performance compared to NPD. There is a normative literature showing that NPD is the best diversification rule investors can implement in practice. DeMiguel, Garlappi, and Uppal

\footnotetext{
${ }^{3}$ For example, in their review paper, Benartzi and Thaler (2007) define the $1 / N$ rule in NPD terms as "when faced with " $N$ "' options, divide asset shares evenly across the options" (p.86). We do not rule out the possibility that the Benartzi and Thaler (2001) results derive from the use of a NPD heuristic in addition to (or even instead of) a NBD heuristic - the empirical context they examine does not disentangle these possibilities. What we can conclude is that in our setting, in which the two types of heuristics can be sharply distinguished, there is evidence of NBD and not NPD.
} 
(2009) evaluate the out-of-sample performance of a suite of models against NPD, including the sample-based mean-variance model, and find that none is consistently better than NPD over reasonable time horizons. ${ }^{4}$ We extend the simulation model used in DeMiguel, Garlappi, and Uppal (2009) to analyze the effectiveness of NBD, and find that NPD outperforms NBD, with the extent of outperformance increasing as the correlation between assets falls and time period increases. In other words, NBD can be costly in practice. Taken together our findings of NBD in stock portfolios and stock-and-fund portfolios, plus the existing evidence for $1 / N$ behavior over longer time horizons in retirement savings schemes (as in Benartzi and Thaler 2001), suggests that the use of NBD is substantially detrimental to investor welfare. ${ }^{5}$

The data we use are provided by a mainstream brokerage platform and cover a large sample of individual investors. We examine the allocation decisions of over 50,000 investors on 260,000 buy-days on which investors purchase multiple stocks. Multiplestock buy-days are important, with one third of the total amount invested in our sample accounted for by multiple-stock buy-day purchases. On approximately one-third of all multiple-stock buy-days investors adopt a NBD heuristic for splitting the funds that they invest, approximately equalizing the value invested in each stock bought on the buy-day.

NBD does not vary by age or gender. NBD might be explained by models of learning and rational inattention, as in the rational inattention approach of Sims (2003). If individual investors face fixed costs of calculating the investment share of each stock in an optimal portfolio (such as time or psychic costs of calculations), then use of NBD would decrease as economic stakes increase. Alternatively, if investors learn portfolio strategies, then use of NBD would decrease with trading experience.

\footnotetext{
4 The reason it is hard to beat this simple heuristic is that there is to high estimation error for portfolio weights estimated from the relatively short-time-period samples available given the fact that means and covariances are not entirely stable over time. Simulations in DeMiguel, Garlappi, and Uppal (2009) show that the estimation window needed for the sample-based mean-variance strategy to out-perform NPD is approximately 3,000 months for a portfolio with 25 assets and approximately 6,000 months for a portfolio of 50 assets.

${ }^{5}$ The inferior portfolio performance of investors in our sample is further exacerbated by the typical portfolio holding only a few stocks (median 5). Goetzmann and Kumar (2008) show that US equity investors on average also hold underdiversified portfolios. Calvet et al. (2007) use comprehensive data from Sweden and show that, while a few households are very poorly diversified, most Swedish households outperform the Sharpe ratio of their domestic stock index through some degree of international diversification.
} 
Our findings provide only a degree of support for an explanation of NBD based upon learning and rational inattention. Consistent with learning, NBD declines with investor experience (measured by account age and frequency of trading). Consistent with rational inattention, NBD declines with financial stakes (measured by investment size and variance in returns on individual stocks bought). ${ }^{6}$ However, NBD is not eliminated even at high levels of investor experience or as the financial stakes of the decisions increase: it is used by investors on more than $20 \%$ of buy-days even at the top of the distributions of the variables by which we measure learning and rational inattention.

Our analysis distinguishes between NBD and NPD. The cleanest context for distinguishing between NBD and NPD is when an investor tops up existing positions in $N$ stocks. For example, an investor holding positions in stocks A and B who, on a buy-day, buys additional units of stock A and stock B. This investor could decide split the new invested funds 50:50 across A and B (i.e., NBD), or split the new funds such that at the end-of-day the ratio of the total market value in A and B is 50:50 (i.e., NPD), or make some other allocation. We find that NBD is overwhelmingly more likely than NPD. Our results show that the rarity of NPD is not due to slow rebalancing, with investors choosing NBD even when NPD is feasible given the amount invested on the buy-day. Additionally, when the analysis is extended to all trades on all buy-days, (and not just top-up days), we find that only very few investors engage in NPD. This evidence indicates that $1 / N$ splitting is almost exclusively a buy-day effect.

Our results focus on how investors frame narrowly on their new purchases within their stock portfolio. Previous studies suggest investors act narrowly across other aspects of their asset allocation, such as retirement saving funds (Choi, Laibson, and Madrian 2009). Hence the narrow framing we observe is most likely a lower bound on the extent of narrow framing in the investor's overall portfolio of assets.

We propose that investors prefer NBD because it simplifies the buying decision problem. The way in which investors implement NBD suggests a strong preference for

\footnotetext{
${ }^{6}$ Also, we find no evidence of day-of-the-week or month-of-the-year differences in the use of NBD. This indicates that NBD is not made more likely by Friday-induced inattention. (Market reactions to earnings announcements are on average more sluggish on Friday afternoons (DellaVigna and Pollet 2009).)
} 
simplicity. Specifically, many investors choose the denominator and numerator of the $1 / N$ calculation to make the division problem simple. When $N=3$, the distribution of total investment amounts is dominated by round number multiples of 3 , with heaping at $£ 3,000$, $£ 6,000, £ 9,000, £ 15,000$ and $£ 30,000$. When $N=4$, the distribution shows heaping at $£ 4,000, £ 8,000, £ 20,000$ and $£ 40,000$. This pattern of investors choosing investment amounts that are round number multiples of the $N$ stocks purchased on the day suggests a strong preference for simplicity in the math of investing. ${ }^{7}$

Overall, these results suggest that the behavior of individual investors is far from optimal. However, the investors in our sample are purchasing multiple stocks and de facto achieving some degree of diversification of their portfolios, albeit in a crude manner. Naïve diversification may be an improvement on no diversification at all; many studies find most investors hold under-diversified portfolios (Barber and Odean 2013). Nevertheless, our results indicate that investors apply the naïve diversification strategy in an extremely narrow way.

\section{Data}

\subsection{Brokerage Account Data}

We use data from Barclays Wealth, a large mainstream UK based broker. The data consist of transaction histories of 182,569 accounts held with the broker between April 2012 and June 2016. The panel data are unbalanced, with accounts opening during the period. Dropping accounts with no activity during the data period, we define the baseline sample as 118,169 accounts that have at least one buy transaction within the data period. The data include stock identification numbers (Stock Exchange Daily Official List (SEDOL) numbers, a list of security identifiers used in the United Kingdom and Ireland for clearing purposes), transaction dates, transaction types (e.g., buy, sell), transaction quantities, and transaction prices. We use SEDOL numbers to match in additional data on individual stock product and performance information via Datastream.

\footnotetext{
${ }^{7}$ Other studies on the role of round numbers in financial markets include Aggarwal and Lucey (2007) and Johnson, Johnson, and Shanthikumar (2007).
} 


\subsection{Sample}

Our interest lies in how individuals choose to allocate funds invested on buy-days involving purchases of multiple stocks. We define a "multiple-stock buy-day" as a day on which the investor makes a purchase of two or more common stocks (denominated in GBP), via either opening a position in new stocks or adding additional shares to an existing position. ${ }^{8}$

Our analysis focuses on two samples. First is a sample of all accounts in which we see at least one multiple-stock buy-day. The sample includes accounts already open at the beginning of our data period and also new accounts which open within the data period. This sample restriction provides 52,866 accounts and 261,585 multiple-stock buy-days. Multiple-stock buy-days account for $30.8 \%$ of the total amount invested over the data period.

Second, from the first sample we draw a sub-sample that includes all new accounts which open within the data period. For this sub-sample of new accounts we have richer data, in that we observe the complete portfolio position of the account from opening date onwards, including for accounts that are transferred in from another broker service. This sample restriction provides 8,982 accounts (43.1\% of new accounts in the baseline sample) and 25,507 multiple-stock buy-days (16.3\% of buy-days in the baseline sample of new accounts).

Approximately $68 \%$ of multiple-stock buy-days involve purchases of two stocks. Among the all accounts sample, $70.4 \%$ of account holders are male. The average age of the account is four years. Account holders make on average 1.8 trades per month, with an average investment amount of over $£ 16,500$ on a multiple-stock buy-day (median value is close to $£ 7,000)$. Among the sample of new accounts, for which we have additional information, investor portfolios are worth on average $£ 61,000$, with an average investment amount on a multiple-stock buy-day of $£ 11,500$. Portfolios contain on average 8 individual stocks and on average investors engage in of 1.5 trades per month. $^{9}$

The low number of individual stocks we see investors in our sample hold in their

\footnotetext{
${ }^{8}$ We restrict to buy-events involving the choice of an investment amount on the part of the investor on the buy-day. Hence we exclude, for example, automatic dividend reinvestments.

${ }^{9}$ Summary statistics are provided in Table A1 and Table A2 in the Online Appendix.
} 
portfolios is consistent with existing studies that document a lack of diversification among individual investors in the individual stock component of the portfolio (Goetzmann and Kumar 2008; Barber and Odean 2013). Purchases of diversified investment products, such as mutual funds or ETFs are rare in the sample - fewer than $6 \%$ of purchases are of mutual funds or ETFs. In addition, while the benefits of diversification increase with the variance in market prices, among the sample of all buy-days (including single-stock buy-days), the average number of stocks purchased per day does not depend on market volatility and does not vary over the sample period. Further details are provided in the Online Appendix.

\section{Naïve Buying Diversification}

\subsection{Multiple-Stock Buy-Days}

We first examine whether investors engage in Naïve Buying Diversification (NBD). We begin by showing allocations across purchased stocks on multiple-stock buy-days. We calculate the percentage of the total buy-day investment (in pounds sterling and net of fees) that is allocated to each stock. ${ }^{10}$ Choosing one of the $N$ stocks at random to be "Stock A", Figure 1 plots the proportion of the buy-day investment allocated to Stock A among all $N$-stock buy-days in the sample, with panels showing different values of $N$. The width of each bin is 0.01 . We focus on allocation to one randomly chosen stock to avoid the dependence of observations that naturally arises because the sum of weights across stocks must be equal to one.

Strikingly, Figure 1 shows large heaping in the frequencies of portfolio weights around $1 / N$. In Panel A, 29.7\% of two-stock buy-days involve allocations of monies invested on the day in the 49-51 interval. This suggests that, as seen in Panel A, many trades are made with allocations that are close to 50:50 and investors may be using that heuristic to guide their allocation choices. In Panels C-D we also see heaping around $1 / N$. On three-stock buy-days, people often spend one third of their money on each stock. On four-stock buy-days, people often spend one quarter on their money on each stock. And so

\footnotetext{
${ }^{10}$ Fees are low as a proportion of the average amount invested. Hence our results are not sensitive to the inclusion of fees in the allocation calculations.
} 
on. ${ }^{11}$

When measuring NBD, we should not restrict the definition to a precise $1 / N$ allocation of funds across $N$ stocks. This is because the indivisibility of individual stocks implies that investors could not in all cases make precise NBD allocations (even if they wanted to). Given the total amount invested and the prices of individual stocks, investors may only be able to achieve an allocation close to $1 / N$. As with most investment platforms, on the Barclays platform investors can input a money amount they would like to invest in a stock and the platform calculates the maximum number of shares (in integers) at the time-limited quoted price (in local currency) that can be purchased with that money amount at the market price. ${ }^{12}$

We therefore present bandwidth measures of NBD. Table 1 reports the proportion of multiple-stock buy-days on which a buy-day investment of $£ P$ is split such that the money value of stock purchases are divided in the intervals $£ P / N \times(1 \pm X)$ where $X$ takes values of $0.02,0.05$ and 0.1 in Panels $\mathrm{A}-\mathrm{C}$. This range of values of $X$ allow for the indivisibility of individual stocks. In the two-stock case, these intervals translate to a proportion of the total buy-day investment invested in Stock A of 49-51 (Panel A), 47.5-52.5 (Panel B) and 45-55 (Panel C). We report 95\% bootstrapped confidence intervals clustered by accounts.

The estimates in Table 1 show that, for two-stock buy-days, allocations fall in the NBD interval in between one quarter and one half of cases, depending on bandwidth. As the number of stocks bought on the multiple stock buy-day increases, the proportion of allocations falling within the range decreases. This will be in part due to a mechanical

${ }^{11}$ We also investigated whether investors might naïvely equate the number of shares purchased across multiple stocks. Additional analysis presented in the Online Appendix demonstrates that this is not the case. Figure A4 Panel A illustrates that allocations in which the number of shares purchased are equalized only occurs when the unit price of the shares is very close. When the unit price of the shares is further apart, we do not see equal allocation of the number of shares, but we do see equal allocation of the money amount (shown in Panel B).

${ }^{12}$ For example, consider the case that an investor intends to invest $£ 1,000$ to buy two stocks, called Stocks $\mathrm{A}$ and B, and that a price of Stock A is $£ 4.50$ per unit and a price of Stock B is $£ 100.50$ per unit. Were the investor to aim for NBD the precise stock split generating an equal money cost split would be: Stock $A=£ 500 / £ 4.50=111.11$ units, Stock B $£ 500 / £ 100.50=4.96$ units. Purchases of common stock must be made in indivisible single units. Due to this indivisibility, the investor cannot invest $£ 500$ for each stock and so might instead decide to buy 111 shares of Stock A with a cost of $111 \times £ 4.50=£ 499.50$ and to buy shares of Stock B with a cost of $5 \times £ 100.50=£ 502.50$. Thus, the allocation of the investment to Stock A is $£ 499.50 \div(£ 499.50+£ 502.50)=.498$ and that to Stock B is .502 . 
effect, as the indivisibility of individual stocks resulting in $1 / N$ allocations being less likely as the number of stocks bought rises. Across all buy-days involving multiple-stock purchases, $49.6 \%$ of investors exhibit at least one buy-day on which they make an allocation in the interval $£ P / N \times(1 \pm X)$ with $X=0.02$.

Our findings raise the question of whether NBD has a mechanical source. For example, if the investment platform uses an interface in which a NBD allocation is presented as an on-screen default, this might lead investors to accept a NBD allocation. However, the platform used by the brokerage did not automatically default, or suggest, equal money investments across multiple stocks. Investors were required to key in their investment amount for each stock separately and each transaction required a separate multiple-screen journey, with no default allocation or recommended allocation shown on screen or in investment guidance. ${ }^{13}$

\subsection{Stock-and-Fund Buy-Days}

We also explore whether investors' use of NBD is associated with a fundamental misunderstanding of the difference between two types of asset categories, individual stocks and funds. In standard portfolio theory there is no reason for the portfolio weights or new purchase weights to be equated between an individual stock and a fund to be anywhere close to equally weighted. We focus on buy-days on which the investor purchased at least one fund and at least one common stock, either by opening a new position or adding to existing position(s). We further restrict our analysis to ETFs, as orders for other types of funds (such as Unit Trusts) are commonly executed with delay of a few days. ${ }^{14}$ Purchases of funds (of any type) are relatively uncommon in the Barclays data. as a result, the sample for this analysis is much smaller than that for the analysis of multiple-stock buy-days.

${ }^{13} \mathrm{~A}$ video showing the Barclays Stockbroking user interface and screen display journey involved in making a purchase can be viewed at https://youtu.be/M1HGgKp6p6k. The video shows that the buy / sell screen display journey shows only information about the stocks to be purchased / sold and does not allow for multiple purchase or sale events in a way that would encourage NBD, or show portfolio information (such as illustration of portfolio positions before or after a purchase / sale) on the same screen.

${ }^{14}$ Orders of other types of funds are queued and batch-processed by the platform every few trading days in order to reduce trading costs. This poses a challenge for our analysis, which focuses on buy-day allocations. This is because in the data we observe the executed price and quantity, which will differ from the order price and quantity placed by the investor a few days previously. We therefore restrict of analysis to ETFs, orders for which are processed in real-time, as is the case for orders of common stocks. 
We adopt the same approach to the calculation of allocations on stock-and-fund buy-days as for multiple-stock buy-days (see Online Appendix for details). We calculate the percentage of the total buy-day investment (in pounds sterling and net of fees) that is allocated to each security. Results reveal that, depending on bandwidth, between one-fifth and one-third of trades involving stock-and-fund combinations have allocations within the $1 / N$ range.

We also explore whether investors demonstrate this same misunderstanding of asset categories when investing in index-ETFs, a category specifically designed to use market weights. We therefore further restrict the sample to FTSE100 ETFs (the two most popular FTSE100 ETFs in the sample (by total holdings) are the ISHARES Core FTSE100 and the Vanguard FTSE100. Results reveal that allocations in the $1 / N$ range are only slightly less common in this subsample: in the two-security case the percentage of index-ETF-and-stock buy-days in the $1 / N$ range is $21.7 \%$ (bandwidth 0.02 ), $26.8 \%$ (bandwidth 0.05 ) and 33.2\% (bandwidth 0.10), compared with 23.0\%, 29.1\% and 36.3\% in the ETF-and-stock buy-day sample. Hence, even among investors who are partly engaged in low-cost index investing, we see similar levels of $1 / N$ allocations compared with those in the multiple-stock and ETF-and-stock buy-day samples. The use of the $1 / N$ heuristic for allocations on stock-and-fund buy-days is particularly striking as it implies, in the two-security example, that the single common stock receives a far higher weighting than the individual holdings within the fund. ${ }^{15}$

\section{Exploring the Sources of NBD}

We now examine whether use of the NBD heuristic varies across investor types. We also examine whether use of the NBD heuristic is consistent with models of learning and rational inattention.

\footnotetext{
${ }^{15}$ For example, the highest weight of any individual stock within a FTSE ETF is approximately $7 \%$. This implies, in the two-security example, that the individual stock would have a weight more than 14 times higher than the largest stockholding within the ETF.
} 


\subsection{Investor Types: Gender and Age}

There is evidence that US males tend to trade more actively and aggressively compared with females (Barber and Odean 2001; Choi et al. 2002; Agnew, Balduzzi, and Sundén 2003; Dorn and Huberman 2005; Mitchell et al. 2006). Consistent with this, we might also expect that men tend to be more confident in choosing portfolio weights. But, alternatively, they might tend more to heuristic thinking, being more likely to follow a gut instinct of choosing $1 / N$. To test between these possibilities, we examine gender subsamples. Figure 2 Panel A shows the bootstrapped estimate of the proportion of two-stock buy-days on which the investment is split within the $49 \%-51 \%$ interval by gender. ${ }^{16}$ The plot shows that the proportions of $1 / N$ buy-day allocations are very similar across men and women.

Previous research has also found that age is associated with lower investment performance, possibly due to age related declines in cognitive abilities (Korniotis and Kumar 2013). Declining cognitive ability might lead older investors to turn to the simple NBD heuristic. Figure 2 Panel B shows the proportion of $1 / N$ buy-days by decade of birth. Very few investors in the sample were born before the 1930s or after the 1990s. The plot illustrates a similar propensity to engage in NBD across investors by age. ${ }^{17}$

\subsection{Do Investors Learn to Avoid Naïve Buying Diversification?}

Previous research offers mixed evidence as to whether investor behavior improves with experience. For example, studies have found that investors learn to avoid the disposition effect as they gain more trading experience (Feng and Seasholes 2005 Seru, Shumway, and Stoffman 2010; ); while in the case of investing in initial public offerings, investors appear to learn to make more mistakes (Kaustia and Knupfer 2008; Chiang et al. 2011). There is evidence that individuals learn from previous experience when using financial products (Agarwal et al. 2008; Ater and Landsman 2013; Miravete and Palacios-Huerta 2014). While

\footnotetext{
${ }^{16}$ We use this interval to identify NBD throughout this section of the paper. Results are not sensitive to widening this interval. Figure A5 shows the similarity in histogram plots for men and women separately, with no notable differences in the distributional characteristics of the buy-day allocation by gender. Table A5 shows summary statistics for the proportion of two-stock buy-days on which the investor uses NBD by investor characteristics.

${ }^{17}$ Online Appendix Figure A6 shows the investor age distribution. Figure A7 shows the similarity in histogram plots for investors by each decade of birth.
} 
a $1 / N$ buy-day allocation might be a naïve action of a new investor, with experienced investors may gain information and feedback that causes them to refine their allocations.

Figure 3 Panel A shows the proportion of NBD buy-days across quartiles of the distribution of account tenure. Measured in months from account opening, the quartile threshold values are 25\%: 24 months; 50\%: 60 months and 75\%: 109 months. Figure 3 shows that the propensity to engage in NBD falls with tenure, though the magnitude of the reduction is small. The proportion of buy-days on which the investor uses NBD falls from $31 \%$ in the bottom quartile to $27 \%$ in the top quartile.

An alternative measure of experience is the frequency with which investors trade, which we measure as the number of trades executed per month. This measure includes all buy and sell trades, whether involving single stock or multiple stocks. A large number of studies have examined the relationship between this measure of trading frequency and performance, typically finding that frequent trading tends to result in lower returns (Barber and Odean 2001). Figure 3 Panel B shows the proportion of NBD buy-days across quartiles of trading frequency. The quartile threshold values of trades per month are $25 \%: 1.1$ trades, $50 \%: 3.1$ trades; $75 \%: 10.2$ trades. As can be seen from the figure, the most frequent traders are much less likely to choose NBD, possibly because some of these individuals are day-traders who very regularly adjust their positions and follow more sophisticated trading strategies. Among the top quartile sub-sample, 19\% of multiple stock buy-days are NBD compared with $36 \%$ in the bottom quartile.

\subsection{Limited Attention Explanations for Naïve Buying Diversification}

Theoretical models have suggested that limited investor attention affects trading and asset prices (Hirshleifer and Teoh 2003; Peng and Xiong 2006; Hirshleifer, Lim, and Teoh 2011). A considerable body of empirical research finds evidence consistent with attention effects in asset markets. ${ }^{18}$ Models of rational inattention explain quasi-rational behaviors as arising due to opportunity costs in allocating attention, as in Sims (2003). Recent studies present mixed evidence as to whether individuals behave in a way that is consistent with

${ }^{18}$ Examples include DellaVigna and Pollet (2009), Hirshleifer, Lim, and Teoh (2009), Hou, Peng, and Xiong (2009), Cohen, Diether, and Malloy (2013) and Huang (2015). 
rational inattention (DellaVigna 2009; Chetty et al. 2014; Taubinsky and Rees-Jones 2018; Gathergood et al. 2019).

In our setting, if investors face fixed costs of calculating the investment share of each stock in a optimal portfolio (such as time or psychic costs of portfolio calculations), then for investment choices with low economic stakes, investors might optimally choose the simple naïve diversification heuristic. In models with fixed optimization costs, it is worth paying the optimization cost (e.g. time cost) only when stakes are sufficiently high. Also, investors may be inattentive to their investment choices when distractions are present (as in Hirshleifer, Lim, and Teoh 2011). This is a feature that DellaVigna and Pollet (2009) refer to as behavioral inattention, who provide evidence of reduced market reaction to earnings announcements made on Fridays. We find no evidence of variation in the use of NBD by day of the week or month of the year. ${ }^{19}$

\subsubsection{Scale of Investment}

To test whether use of the NBD heuristic is consistent with limited attention, we first examine the relationship between NBD and the financial stakes of the investment decision.

The financial stakes of the investment allocation choice can be defined in two main dimensions - the difference in returns among the stocks bought and the total value of the buy-day investment. When returns and risks differ more across stocks, optimal allocations tend to differ more from NBD. We explore how NBD buy-days relate to the difference in past returns, or the difference in future returns, between the two stocks purchased on the two-stock buy-day. We use the simple difference in gross returns over the previous 3 months, excluding dividend payments, capital gain taxes, or trading fees. We then separate the sample into quartiles by the difference in returns. ${ }^{20}$

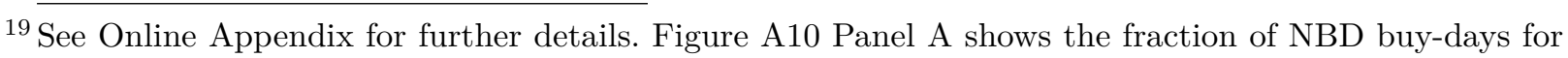
each day of the week. Figure A11 shows the distribution of trades by day of the week and Figure A13 shows the distribution of trades by month of the year. Figure A12 confirms that the histogram plots are very similar for each day of the week. Figure A10 Panel B shows the fraction of NBD buy-days for each month of the year. Figure A14 confirms that the histogram plots are very similar for each month of the year. Table A6 Panel A reports supporting summary statistics.

${ }^{20}$ It is possible that investors might be more likely to use NBD when stocks have similar characteristics, such as being from firms in the same industry. However, additional analysis in Online Appendix Figure A17 shows that this is not the case. Panel A shows that investors are as likely to engage in NBD when buying two stocks from firms in the same industry as they are when buying two stocks from firms in different
} 
Figure 3 Panel $\mathrm{C}$ shows the proportion of $1 / N$ buy-days for past returns. The proportion of buy-days with NBD declines with the difference in past returns and is notably lower in the top quartile sub-sample compared with the third quartile. Approximately $24 \%$ of buy-days in the top quartile are NBD, compared with $33 \%$ in the bottom quartile. A similar pattern in seen in Panel D for future returns, in which approximately $25 \%$ of buy-days in the top quartile are NBD compared with $32 \%$ in the bottom quartile.

Panels E and F of Figure 3 illustrate the relationship between NBD behavior and the total value of the investor's portfolio with the brokerage (Panel E) and the amount invested on the day (Panel F). Panel E illustrates that investors with high value portfolios are less likely to adopt a NBD heuristic. ${ }^{21}$

The total amount invested on the day is the simple sum of the two-stock purchases. Figure 3 Panel F illustrates the proportion of buy-days with NBD across quartile subsamples. Among the sample the median investment amount on the day is $£ 5,200$. Figure 3 Panel F shows the proportion of buy-days exhibiting NBD falls as the investment amount increases. At the top quartile $24 \%$ of buy-days involve a $1 / N$ allocation, compared with $35 \%$ in the bottom quartile. However, Panel $\mathrm{F}$ also illustrates a non-monotonic relationship, with the proportion increasing between the second and third quartiles, before falling at the fourth quartile. ${ }^{22}$

Later, we show that this pattern arises due to investors making joint decisions over their investment amount on the day and the number of stocks bought. This results in high value investment amounts (e.g. at higher round number multiples of 2 such as $£ 10,000$ and $£ 20,000$ ) being more popular among investors using NBD compared with lower value investment amounts.

industries.

${ }^{21}$ This analysis is only feasible for new accounts, as we can only build complete portfolios from transaction data for new accounts, as old accounts have a composition which is not seen by us at the beginning our of data period. We replicate our main result among all new accounts in Online Appendix Figure A18, which shows that approximately $34 \%$ of two-stock buy-days are made in the $49 \%-51 \%$ interval among the sample of new accounts.

${ }^{22}$ Additional analysis in the Online Appendix in Figure A20 shows the histogram plots and confirms this non-monotonic pattern, which is attributable to less bunching at the centre of the distribution in the second quartile. 


\subsubsection{Trading Activity and Portfolio Size}

In a limited attention setting there may be a one-time fixed cost of adopting a decision making strategy (e.g., learning how to use an online portfolio optimizer). The returns from adopting a decision making strategy are higher as portfolio size and trading activity increase. Hence, use of NBD will fall with trading activity and portfolio size.

Figure 4 Panel A shows the proportion of NBD buy-days by whether the investor holds existing positions in their portfolio and Panel B by whether the investor also makes a sale on the buy-day. The panels show the proportion of NBD buy-days is lower with existing positions held (31\% compared with 39\%) and with sales on the day (22\% compared with $35 \%$ ).

Finally, we explore the relationship between NBD behavior and the number of stocks in the individual investors' portfolio. It is well documented that individual investors tend to hold only a few stocks as direct holdings in their portfolios (Barber and Odean 2013). In our data the cut points for quartiles of the number of stocks held are 25\%: 3, 50\%: 5 and 75\%: 9. Figure 4 Panel $\mathrm{C}$ shows the proportion of NBD buy-days by number of stocks held. There is no decline in the proportion of NBD buy-days with the number of stocks held.

Overall, these results suggest that the use of the NBD heuristic decreases both with investor experience and as the incentives to allocate attention increase. Nevertheless, among top quartile of buy-days by financial characteristics (including portfolio value, number of stocks in the portfolio and differences in returns across stocks) the proportion of investments made with NBD is consistently above $20 \%$. Hence NBD is only modestly sensitive to the economic stakes of investing.

\subsection{Taking Stock of NBD Behavior}

To explore the relative importance of the multiple possible sources of NBD behavior, we estimate cross-section multivariate regression models. Of course, these estimates do not establish causality. In the probit model the dependent variable is a dummy variable indicating whether the two-stock buy-day allocation is NBD, again using the $£ P / N \times$ 
$(1 \pm 0.02)$ interval to define an approximate NBD. The model is estimated on the sample of all multiple-stock buy-days from the new accounts sample.

Results are shown in Table 2. Model 1 includes investor gender dummies, decade of birth dummies, day of the week dummies and month of the year dummies (coefficients not shown), plus an intercept term. Consistent with the unconditional relationships shown above, none of the coefficients returned in the regression are statistically significantly different from zero. Model 2 adds dummies for account tenure quartiles and a continuous measure of the average number of trades per month. The coefficient on the average number of trades per day is negative and statistically significant. Model 3 adds the additional variables. Results show the likelihood of a NBD declines with the number of stocks bought on the day, the range in future returns of stocks in the portfolio, whether the investor holds an existing position in their position and whether a sale is made on the same day.

\section{Naïve Buying Diversification or Naïve Portfolio Diversification?}

A key contribution of our paper is to distinguish whether investors engage in NPD (targeting equal weights in the portfolio) or NBD (targeting equal weights in a given purchase). This distinction has important welfare consequences. As a target portfolio-balancing heuristic, NPD arguably performs well for investors in practice. From a normative perspective, DeMiguel, Garlappi, and Uppal (2009) compare the performance of NPD against 14 alternative models and find that none is consistently better than NPD in achieving a Sharpe ratio, certainty-equivalent return or turnover.

\subsection{Evidence from Top-Up Buy-Days}

The cleanest setting in which to distinguish between NBD and NPD is when investors top-up multiple stocks already held in their portfolio. We examine whether they split the top-up investment $1 / N$ across new funds invested (i.e., NBD), or instead top up such that the portfolio is balanced $1 / N$ as a result of the trades (i.e., NPD).

First we show results for top-up buy-days involving two stocks. Panel A of Figure 5 
shows the proportion of the buy-day investment allocated to (randomly chosen) Stock A. The right-side figure shows the market value of Stock A over the total end-of-day portfolio value (of holdings of Stocks $\mathrm{A}$ and $\mathrm{B}$ ). There is clear heaping around $1 / N$ in the left-side figure, which is absent in the right-side figure. Hence in this sample we see NBD, not NPD, on top-up buy-days.

Investors may fail to achieve NPD simply because the level of total investment on the buy-day is not large enough to bring their portfolio into balance. In that case, an investor could only achieve NPD if they increased the total amount invested on the buy-day, or reduced their holdings of one position. In Panel B of Figure 5 we restrict the sample to top-up buy-days on which the investor could achieve NPD, given the total buy-day investment amount, without requiring any sell activity. Again, there is clear heaping around $1 / N$ in the left-side figure, which is absent in the right-side figure.

Next we extend our analysis to all top-up buy-days involve multiple stocks. Results are shown in Table 3, which presents a breakdown of the starting positions, buying allocations, and ending positions of all buy-day episodes with at least two existing positions in the portfolio. In each panel the rows summarize eight mutually exclusive scenarios for account positions at the start of the day and activity during the day.

The first four rows in each panel show accounts which begin the buy-day with NPD (2.2\% of observations). Of these, $1.1 \%$ engage in NBD and $1.1 \%$ engage is non-NBD. At the day end, in $0.5 \%$ of cases the portfolio is balanced by NPD in $1.9 \%$ of cases the portfolio is not balanced by NPD. The bottom four rows in each panel show accounts which begin the buy-day away from NPD (97.8\% of observations). Of these, in $28.5 \%$ of cases investors use NBD and $69.2 \%$ of cases they choose some other allocation. In only $0.2 \%$ of cases the portfolio shows NPD. Panels B and C show similar results. Table 4 shows the proportion of buy-days resulting in NPD in the restricted sample. Across all multiple-stock buy-days in the restricted sample, only $2 \%$ result in NPD.

In summary, this evidence indicates that investors are prone to naive buying diversification. When investors hold multiple-stocks in their portfolio and make top-up purchases of the same stocks on the buy-day, they are many times more likely to engage 
in NBD than NPD.

\subsection{Evidence from All Buy-Days}

To verify whether this finding of naive buying diversification generalizes beyond this specific sample conditioning, we now extend the analysis to examine purchases without restricting either the numbers of stocks currently held nor the set of stocks purchased on the buy-day.

The panels in Figure 6 describe behavior for different numbers of positions in the investor's existing portfolios at the beginning of the buy-day. Within each panel, the horizontal axis denotes the number of stocks bought on the day. The red bars and whiskers illustrate $95 \%$ confidence intervals for the proportion of buy-days within the cell that show NBD. The blue bars and whiskers illustrate $95 \%$ confidence intervals for the proportion of buy-days that show NPD.

Strikingly, NBD is consistently high on buy-days in which, at the beginning of the day, there are multiple stocks in the portfolio. In contrast, NPD is rare across all combinations of existing positions and numbers of stocks bought on the day (with the exception of empty accounts with no existing positions at the beginning of the day for which, by construction, NBD $=$ NPD). Table 5 and Table 6 report the mean values and bootstrapped $95 \%$ confidence interval bounds within each cell. This analysis makes clear that NPD is extremely rare, whether investors are topping-up their portfolios or adding new positions.

\section{Naïve Buying Diversification and Portfolio Performance}

Our main result, that investors often use NBD, whereas there is no evidence that they use NPD, raises the question of whether, and how much, NBD damages portfolio performance compared to NPD. There is evidence that owing to estimation problems for investors, the NPD rule is the best diversification rule that investors can implement in practice. As mentioned earlier, DeMiguel, Garlappi, and Uppal (2009) evaluate the out-of-sample performance of 14 different portfolio estimation and formation models against NPD, and 
find that none perform consistently better than NPD over reasonable time horizons. We therefore compare the performance of NBD with that of NPD. Our main result is that NPD outperforms NBD when the correlation in returns across assets is low (i.e., idiosyncratic risk is high). We briefly outline here the simulation model that we use to compare the performance of NPD and NBD. Further details are provided in Appendix A.

Simulated asset returns are generated according to the single-factor model used in DeMiguel, Garlappi, and Uppal (2009). We assume that an investor $i$ holds a portfolio consisting of $N_{i}$ risky assets, one of which is the factor. Annualized excess returns of the factor, $R_{b, t}$, are drawn from the normal distribution $R_{b, t} \sim N\left(\mu_{b}, \sigma_{b}^{2}\right)$. Annualized excess returns of the remaining $N_{i}-1$ risky assets, $R_{a, t}$, follow $R_{a, t}=B R_{b, t}+\epsilon_{t}$, where $B$ is the factor loading and $\epsilon_{t}$ is an error term drawn from the normal distribution $\epsilon_{t} \sim N\left(0, \Sigma_{t}\right) . \Sigma_{t}$ is a diagonal variance-covariance matrix where the squared root of the diagonal elements represent the idiosyncratic volatility.

We implement the NBD and NPD rules over a time horizon $T$ (months) with a regular contribution into the portfolio at each time step. Under the NPD rule, assets are sold and purchased at each time step to rebalance the portfolio to $1 / N_{i}$ weights. Under the NBD rule, the monthly contribution is simply split equally over the $N_{i}$ assets. We evaluate portfolio performance using the Sharpe ratio based on 1,000 simulations.

We present two calibrations, one with low and one with high idiosyncratic volatility (the squared root of the diagonal elements of $\Sigma_{t}$ ). These are calibrated to the inter-quartile range $(25 \%-75 \%)$ of estimated annualised idiosyncratic volatilities of stocks in the data (other parameter values used in the simulation are the same as those in DeMiguel, Garlappi, and Uppal (2009): $\mu_{b}=0.08$ and $\sigma_{b}=0.16, B$ are evenly spread from 0.5 to 1.5 .) In the low idiosyncratic risk simulation the idiosyncratic components are drawn from a $U[0.10,0.30]$, which is shifted to $U[0.40,0.60]$ in the high idiosyncratic risk simulation. ${ }^{23}$ We conduct

${ }^{23}$ We fitted daily excess returns of ordinary shares listed in the London Stock Exchange to the single-index model where the index is FTSE 500 index. (Note that we used 200 stocks which were traded throughout the data period and were most frequently traded in the data. Stocks having a zero daily return for more than $25 \%$ of trading days were excluded. As a risk-free rate, we used the UK government liability 1 year spot rate retrieved from https://www.bankofengland.co.uk/statistics/yield-curves.) The inter-quartile range $(25 \%-75 \%)$ of the estimated annualized idiosyncratic volatilities is approximately 0.20 (the average in the low idiosyncratic risk simulation) and 0.50 (the average in the high idiosyncratic risk simulation), respectively. Idiosyncratic volatilities of $56 \%$ of stocks are within the parameter value range in the low 
simulations for each pairwise combination of $N_{i} \in\{5,10,20\}$ and $T \in\{120,360\}$.

Table 7 summarizes results from the simulation exercise. In the low idiosyncratic risk simulations, shown in Panel A, a t-test fails to reject the null hypothesis that the average Sharpe ratios of the NBD and NPD strategies are equal. The NPD simulations in Panel A are consistent with those in Table 6 of DeMiguel, Garlappi, and Uppal (2009).

In contrast, the high idiosyncratic risk simulations, shown in Panel B, NBD substantially reduces Sharpe ratios relative to NPD, and the $t$-test rejects the null hypothesis of equality in the Sharpe ratios at the $1 \%$ level in the majority of cases. The difference in Sharpe ratio increases with both $N_{i}$ and $T$. The reduction in Sharpe ratio under the NBD rule compared with the NPD ranges from $11.0 \%$ (with $N_{i}=5$ and $T=120$ ) to $22.7 \%$ (with $N_{i}=20$ and $T=360$ ).

Intuitively, NBD tends to induce arbitrary disparities in portfolio weights, so we do not expect it to do as good a job as NPD in evenly balancing the portfolio and diversifying risk. ${ }^{24}$ Such imbalance tends to increase portfolio risk more when there is greater idiosyncratic risk to be diversified. Furthermore, portfolio weights tend to diverge as random return realizations shift relative asset values. $T$ and this random divergence is stronger when return correlations are low (i.e., idiosyncratic risk is high). Such divergence can create especially large non-diversification for portfolios that are already imbalanced, as arises under NBD, because random divergence in the weights in the few stocks that are most heavily held has a larger effect on portfolio volatility. In contrast, NPD adjusts portfolio weights to offset random divergence. These adverse effects for NBD are increasing with both $T$ and $N_{i}$, with higher $T$ allowing further random divergence from initial weights and higher $N_{i}$ generating lower correlation in returns.

idiosyncratic risk simulation (0.10-0.30) and those of $17 \%$ of stocks are within the parameter value range in the high idiosyncratic risk simulation (0.40-0.60).

${ }^{24}$ For example, in general an asset that happens to be purchased on a day when few other assets are purchased or on a day when a greater amount of funds are invested will tend to get higher portfolio weight. Also, an asset that happens to be bought on multiple days will tend to get higher portfolio weight. 


\section{7. $1 / N$ Selling}

Investors could employ a Naïve Selling Diversification (NSD) heuristic when selling multiple stocks. However, if NBD is due to narrow framing, then NSD might be less likely, as when making selling decisions investors are confronted with information on their portfolio. Previous research suggests investor selling behavior is related to the framing of the portfolio, including the well-known disposition effect (Barber and Odean 2013) and rank effects (Hartzmark 2015). By these heuristics, investors tend to pick out stocks from the distribution within their portfolio. Indeed, in our data the vast majority of sell-days involve single stocks. We observe 1,108,080 sell-days, among which $84 \%$ are sell-days involving a single-stock sale. The majority of sell trades are liquidating sales $(62 \%)$ with the remainder partial sales. (Another category is short-selling, which is uncommon in our data, affecting only $7 \%$ of sell-trades.)

\section{1. $1 / N$ Selling on Multiple-Stock Sell Days}

Additional analysis in the Online Appendix illustrates the proportion of selling proceeds from a randomly chosen stock, Stock A, on two-stock sell-days. The peak at $1 / N$ accounts for approximately $10 \%$ of sell-days in the $49 \%-51 \%$ interval. In total only $6.2 \%$ of all multiple-stock sell-days involve investors choosing NSD. Overall, NSD accounts for fewer than $1.5 \%$ of all sell-days in the data.

\subsection{Selling to Achieve $1 / N$ Portfolios}

Alternatively, investors might sell positions such that they achieve NPD. Again, this might be more likely than NPD when buying because selling stocks necessarily involves looking at the values of existing positions. We calculated the percentage of sell-days that result in NPD by combinations of the number of existing positions in the portfolio at the start of the day, number of positions sold (either partially or fully liquidated) and number of resulting positions. Results show that NSD is a very rare outcome. ${ }^{25}$

\footnotetext{
${ }^{25}$ When we restrict the sample to sell-days on which the investor can achieve NSD without requiring any purchases of stocks, we find that NSD is achieved on only $7.2 \%$ of sell-days (see Online Appendix Table A10). When we restrict the sample to days with both buy and sell trades on which the investor
} 


\section{Do Investors Jointly Choose Investment Amounts and N?}

A possible motivation for using the NBD heuristic is that it simplifies the decision problem. If so, we would expect that investors who use the NBD heuristic would implement it in a simple way. To investigate this, we analyze how investors choose total amount to invest on the day and the number of stocks bought $(N)$. We find that they choose these in order to make the division calculation simpler, say choosing to invest approximately $£ 15,000$ in three stocks or $£ 10,000$ in two stocks. This is consistent with NBD investors having a preference for simplicity.

In Figure 7 we plot the investment amount on multiple-stock buy-days on which individuals split their purchases $1 / N$, for different value of $N$. The striking feature of the plot is the heaping of investment amounts around simple round-number multiples of $N$. Beginning at Panel A with $N=2$, one observes heaping at values of $£ 1,000, £ 2,000$, $£ 4,000, £ 10,000$ and $£ 20,000$. By contrast, in Panel B with $N=3$ we see investment amounts dominated by numbers which are simple multiples of $3, £ 1,500, £ 3,000, £ 6,000$, $£ 9.000, £ 15,000$ and $£ 30,000$. We further see this patterns when $N=4$ and when $N=5$. In Panel C, showing $N=4$, we see heaping at $£ 2,000, £ 4,000, £ 8,000, £ 10,000, £ 12,000$, $£ 20,000$, and $£ 40,000$. In Panel $\mathrm{D}$, showing $N=5$, we see heaping at at $£ 2,500, £ 5,000$, $£ 10,000, £ 25,000$ and $£ 50,000$. It is further striking that the modal investment bin is $£ 2,000$ when $N=2, £ 3,000$ when $N=3, £ 4,000$ when $N=4$ and $£ 5,000$ when $N=5$. One outcome of this behavior is to give rise to near-identical distributions of investment amounts per stock across multiple-stock buy-days involving two - five stocks. The distribution of investment amount per stock across five-stock buy-days appears nearidentical to that across two-stock buy-days. Hence the total amount invested on the buy-day rises monotonically with the number of stocks bought, while the average amount invested in each stock remains constant. However, while the average amount invested in each stock remains constant with the number of stocks in the sample, this is not true at the investor level. Restricting to the sample of investors who make at least one multiple-stock buy-trade

can achieve NSD by reallocating the total sales and total investment on the day without requiring any additional purchase or sale, we find that NSD is achieved on only $1.6 \%$ of sell-days (see Table A11). 
and one single-stock buy-trade within the sample period, only $2.3 \%$ of investors spend approximately the same amount on every trade (allowing a 10\% bandwidth). Therefore, we can rule out the hypothesis that NBD arises due to a coincidence of investors always investing a constant amount per stock and sometimes buying multiple stocks on the same day.

An example of a compelling pattern pointing in this direction is the observation that investors tend to buy 2 not 3 stocks with a spend of $£ 2,000,3$ not 2 stocks with a spend of $£ 3,000$, but then often 2 not 3 stocks with a spend of $£ 4,000$, suggesting that the total sum of money available for investment may be determining the number of stocks bought in a very non-monotonic way. An interpretation of these results is that investors are not only utilizing $1 / N$ as a simple heuristic for allocating across $N$ stocks, but that they are choosing a total investment amount to be allocated such that $1 / N$ becomes a simple calculation although we do not have experimental or natural sources of exogenous variation in either the total investment amount or $N$ within or across investors.

\section{Conclusion}

We investigate how investors go about approaching a common financial choice: allocating invested funds across multiple stocks bought on the same day. Previous research on retirement saving fund allocation has proposed that investors use a $1 / N$ heuristic, but has not disentangled whether individuals use $1 / N$ as a rule for dividing the amount invested across funds, or as a target portfolio allocation. We disentangle these, showing that a common approach among investors is to simplify this problem by applying a $1 / N$ heuristic to their buy-day purchases, approximately equalizing the money amount invested across several stocks purchased on the day or across a mixture of stocks and funds, a behavior which we term Naïve Buying Diversification (NBD). In contrast, investors almost never achieve approximately equal money amounts invested in each stock, an outcome we term Naïve Portfolio Diversification (NPD)

The propensity to use the NBD heuristic decreases with investor experience and financial stakes, consistent with models of learning and attention allocation. Nevertheless, 
even when investor experience is high and financial stakes are large, the proportion of investments made with NBD is above $20 \%$. Hence NBD is only modestly sensitive to investor experience and economic stakes of investing. Investors may use the NBD heuristic because of an attraction to simplicity. Consistent with this, investors implement NBD in a simple way, appearing to choose both margins in order to make the $1 / N$ task mathematically simple.

Use of the NBD heuristic has the result of creating portfolio shares for individual stocks that do not closely approach equalizing weights in the investor's overall portfolio (Naïve Portfolio Diversification). This behavior is consistent with narrow framing, whereby investors appear to approach the buy-day task of allocating funds across stocks in isolation from their existing portfolio positions. In other words, they engage in transactional framing instead of portfolio framing.

Barberis and Huang (2001) argue that when an investor contemplates adding an incremental gamble the the investor's portfolio, the investor narrowly frames on the increment rather than on the overall portfolio; and that this offers an explanation for otherwise puzzling phenomena, such as the rejection of small independent gambles, and the stock market participation puzzle. We show that the tracks of such narrow framing are evident in the incremental purchases of assets already in the portfolio. The resulting $1 / \mathrm{N}$ behavior makes it especially clear that investors are engaged in narrow framing on the incremental purchase. Even conditional on making an asset purchase, narrow framing on the transaction results in bad choices of the relative weights allocate to the different securities in the transaction. So we document that narrow framing extends to incremental portfolios of gambles, not just single ones. This suggests that behavioral models of investment decisions should incorporate the effects of transactional framing by investors.

Our findings also have implications for the design of contribution-based savings plans, such as pension schemes. Such schemes typically ask investors to select buying weights across funds for continuing monthly contributions. This can have the adverse sideeffect of encouraging investors to use the NBD heuristic without integrated consideration 
of the current portfolio allocation. Our findings suggest that a different scheme may help investors achieve better-diversified overall portfolios. Such a scheme could offer investors a choice over overall portfolio target. The scheme would then automatically provide the investor simultaneously with (1) a recommended portfolio reallocation, and (2) monthly contribution weights consistent with this target. An interesting topic for future research is whether such a scheme improves investor diversification and portfolio performance. 


\section{References}

Agarwal, S., J. C. Driscoll, X. Gabaix, and D. Laibson (2008). Learning in the Credit Card Market. NBER Working Paper 13822.

Aggarwal, R. and B. Lucey (2007). Psychological Barriers in Gold Prices? Review of Financial Economics 16, 217 - 230.

Agnew, J., P. Balduzzi, and A. Sundén (2003). Portfolio Choice and Trading in a Large 401(k) Plan. The American Economic Review 93, 193-215.

Ater, I. and V. Landsman (2013). Do Customers Learn from Experience? Evidence from Retail Banking. Management Science 59, 2019-2035.

Barber, B. M. and T. Odean (2000). Trading Is Hazardous to Your Wealth: The Common Stock Investment Performance of Individual Investors. Journal of Finance 55, 773-806.

Barber, B. M. and T. Odean (2001). Boys will be Boys: Gender, Overconfidence, and Common Stock Investment. The Quarterly Journal of Economics 116, 261-292.

Barber, B. M. and T. Odean (2013). The Behavior of Individual Investors. Handbook of the Economics of Finance; Volume 2A (1), 1533-1570.

Barberis, N. (2018). Psychology-based models of asset prices and trading volume. In Handbook of Behavioral Economics: Applications and Foundations 1, Volume 1, pp. 79-175. Elsevier.

Barberis, N. and M. Huang (2001). Mental Accounting, Loss Aversion and Individual Stock Returns. Journal of Finance 56, 1247-1292.

Barberis, N. and M. Huang (2008). The Loss Aversion / Narrow Framing Approach to the Equity Premium Puzzle. In R. Mehra (Ed.), Handbook of the Equity Risk Premium, Chapter 6. Elsevier.

Barberis, N., M. Huang, and D. Thaler (2006). Individual Preferences, Monetary Gambles, and Stock Market Participation: A Case for Narrow Framing. American Economic Review 96, 1069-1090.

Barberis, N., A. Mukherjee, and B. Wang (2016). Prospect Theory and Stock Returns: An Empirical Test. Review of Financial Studies 29, 3068-3107.

Benartzi, S. and R. Thaler (2001). Naive Diversification Strategies in Defined Contribution Saving Plans. American Economic Review 91, 79-98.

Benartzi, S. and R. Thaler (2007). Heuristics and Biases in Retirement Savings Behavior. Journal of Economic Perspectives 21, 81-104. 
Calvet, L. E., J. Y. Campbell, and P. Sodini (2007). Down or Out: Assessing the Welfare Costs of Household Investment Mistakes. Journal of Political Economy 115(5), 707-747.

Chetty, R., J. N. Friedman, S. Leth-Petersen, T. H. Nielsen, and T. Olsen (2014). Active vs. Passive Decisions and Crowd-Out in Retirement Savings Accounts: Evidence from Denmark. The Quarterly Journal of Economics 129, 1141-1219.

Chiang, Y.-M., D. Hirshleifer, Y. Qian, and A. E. Sherman (2011). Do Investors Learn from Experience? Evidence from Frequent IPO Investors. Review of Financial Studies 24, $1560-1589$.

Choi, J., D. Laibson, and B. Madrian (2009). Mental Accounting in Portfolio Choice: Evidence from a Flypaper Effect. American Economic Review 99, 2085-2095.

Choi, J., D. Laibson, B. Madrian, and A. Metrick (2002). Defined Contribution Pensions: Plan Rules, Participant Choices, and the Path of Least Resistance. In R. Moffitt (Ed.), Tax Policy and the Economy, Volume 16, pp. 67-114.

Cohen, L., K. Diether, and C. Malloy (2013). Misvaluing Innovation. The Review of Financial Studies 26, 635-666.

Daniel, K. D., D. Hirshleifer, and A. Subrahmanyam (2001). Overconfidence, Arbitrage, and Equilibrium Asset Pricing. Journal of Finance 56, 921-965.

DellaVigna, S. (2009). Psychology and Econmics: Evidence From the Field. Journal of Economic Literature 47, 315-372.

DellaVigna, S. and J. M. Pollet (2009). Investor Inattention and Friday Earnings Announcements. Journal of Finance 64, 709-749.

DeMiguel, V., L. Garlappi, and R. Uppal (2009). Optimal Versus Naive Diversification: How Inefficient is the 1/ N Portfolio Strategy? Review of Financial Studies 22, 1915-1953.

Dorn, D. and G. Huberman (2005). Talk and Action: What Individual Investors Say and What They Do. Review of Finance 9, 437-481.

Feng, L. and M. S. Seasholes (2005). Do Investor Sophistication and Trading Experience Eliminate Behavioral Biases in Financial Markets? Review of Finance 9, 305-351.

Gathergood, J., N. Mahoney, N. Stewart, and J. Weber (2019). How Do Individuals Repay Their Debt? The Balance-Matching Heuristic. American Economic Review 109, 844-875.

Gilovich, T. and D. Griffin (2010). Judgment and Decision Making. In S. T. Fiske, D. T. Gilbert, and G. Lindzey (Eds.), Handbook of Social Psychology Vol. 2, Chapter 15, pp. 576. John Wiley \& Sons. 
Goetzmann, W. N. and A. Kumar (2008). Equity Portfolio Diversification. Review of Finance 12, 433-463.

Grinblatt, M. and B. Han (2005). Prospect Theory, Mental Accounting, and Momentum. Journal of Financial Economics 78, 311-339.

Hartzmark, S. M. (2015). The Worst, the Best, Ignoring All the Rest: The Rank Effect and Trading Behavior. Review of Financial Studies 28, 1024-1059.

Hirshleifer, D., S. Lim, and S. Teoh (2009). Driven to Distraction: Extraneous Events and Underreaction to Earnings News. The Journal of Finance 64, 2289-2325.

Hirshleifer, D., S. S. Lim, and S. H. Teoh (2011). Limited Investor Attention and Stock Market Misreactions to Accounting Information. The Review of Asset Pricing Studies 1, $35-73$.

Hirshleifer, D. and S. H. Teoh (2003). Limited Attention, Information Disclosure, and Financial Reporting. Journal of Accounting and Economics 36, 337-386.

Hirshleifer, D. A. (2015). Behavioral Finance. Annual Review of Financial Economics 7, 133-159.

Hou, K., L. Peng, and W. Xiong (2009). A Tale of Two Anomalies: The Implications of Investor Attention for Price and Earnings Momentum. Working Paper.

Huang, X. (2015). Thinking Outside the Borders: Investors' Underreaction to Foreign Operations Information. The Review of Financial Studies 28, 3109-3152.

Huberman, G. and W. Jiang (2006). Offering versus Choice in 401(k) Plans: Equity Exposure and Number of Funds. Journal of Finance 61, 763-801.

Johnson, E., N. B. Johnson, and D. Shanthikumar (2007). Round Numbers and Security Returns. Working Paper.

Kaustia, M. and S. Knupfer (2008). Do Investors Overweight Personal Experience? Evidence from IPO Subscriptions. Journal of Finance 63, 2679-2702.

Korniotis, G. M. and A. Kumar (2013). Do Portfolio Distortions Reflect Superior Information or Psychological Biases? Journal of Financial and Quantitative Analysis 48, $1-45$.

Li, Y. and L. Yang (2013). Prospect Theory, The Disposition Effect, And Asset Prices. Journal of Financial Economics 107, 715-739.

Madrian, B. and D. Shea (2001). The Power of Suggestion: Inertia in 401(k) Participation and Savings Behavior. Quarterly Journal of Economics 4, 1149-1187. 
Markowitz, H. (1952). Portfolio Selection. Journal of Finance 7, 77-91.

Miravete, E. J. and I. Palacios-Huerta (2014). Consumer Inertia, Choice Dependence, and Learning from Experience in a Repeated Decision Problem. Review of Economics and Statistics 96, 524-537.

Mitchell, O. S., G. R. Mottola, S. P. Utkus, and T. Yamaguchi (2006). The Inattentive Participant: Portfolio Trading Behavior in 401(K) Plans. SSRN Electronic Journal.

Odean, T. (1998). Are Investors Reluctant to Realize Their Losses? Journal of Finance 53, $1775-1798$.

Peng, L. and W. Xiong (2006). Investor Attention, Overconfidence and Category Learning. Journal of Financial Economics 80, 563-602.

Seru, A., T. Shumway, and N. Stoffman (2010). Learning by Trading. Review of Financial Studies 23, 705-739.

Sims, C. A. (2003). Implications of Rational Inattention. Journal of Monetary Economics 50, 665-690.

Taubinsky, D. and A. Rees-Jones (2018). Attention Variation and Welfare: Theory and Evidence from a Tax Salience Experiment. The Review of Economic Studies 85, 2462 2496. 
Figure 1: Proportion of Buy-Day Investment Allocated to Each Stock on Multiple-Stock Buy-Days

(A) Two Stocks

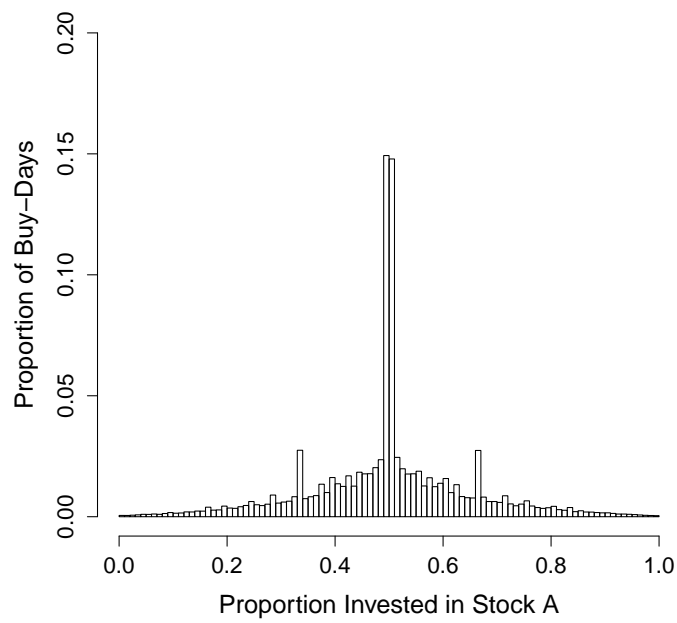

(C) Four Stocks

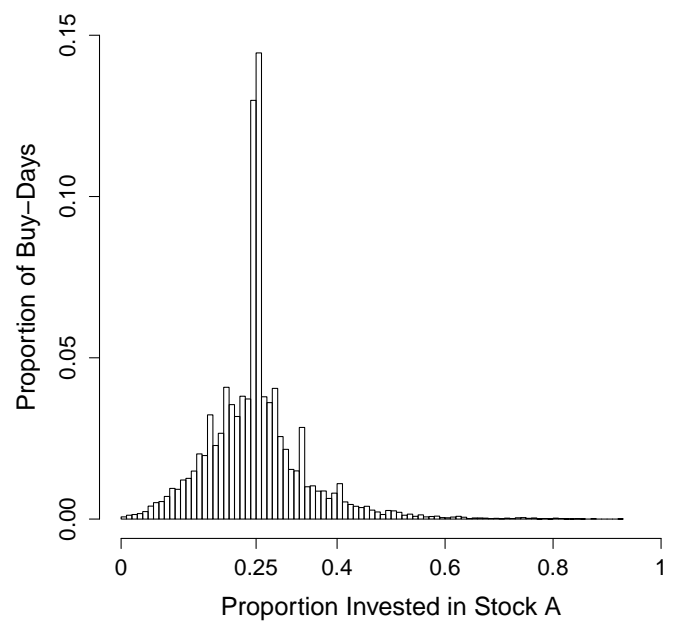

(B) Three Stocks

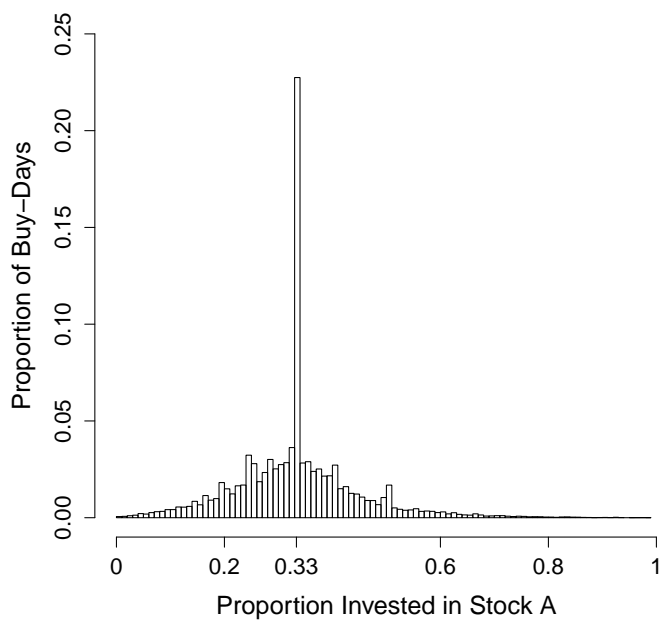

(D) Five Stocks

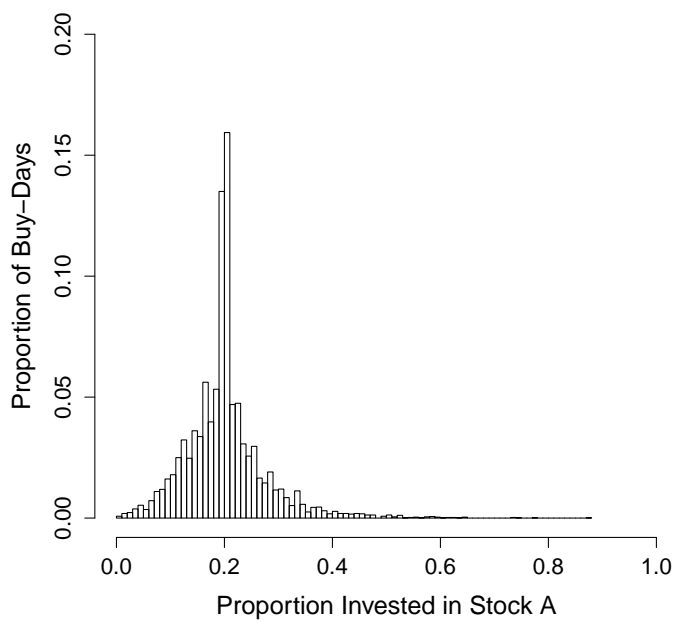

Note: Figure shows a histogram of the proportion of the total buy-day investment (in pounds) which is invested in Stock A, where Stock A is a randomly chosen stock from the group of stocks purchased. Bin width is 0.01. Sample is restricted to multiple-stock buy-days. See Section 2 for details on the sample construction. 
Figure 2: Naïve Buying Diversification by Gender and Age Two-Stock Buy-Days

(A) Gender

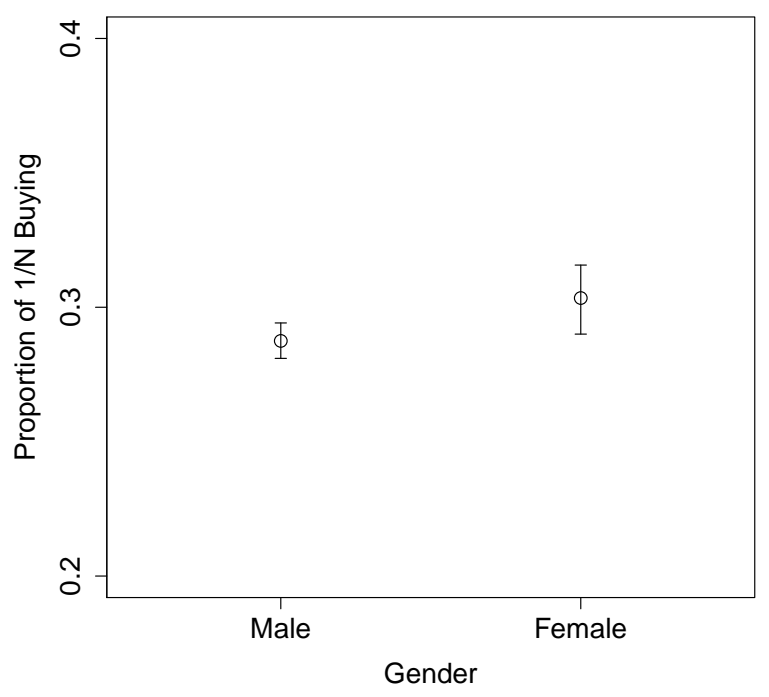

(B) Decade of Birth

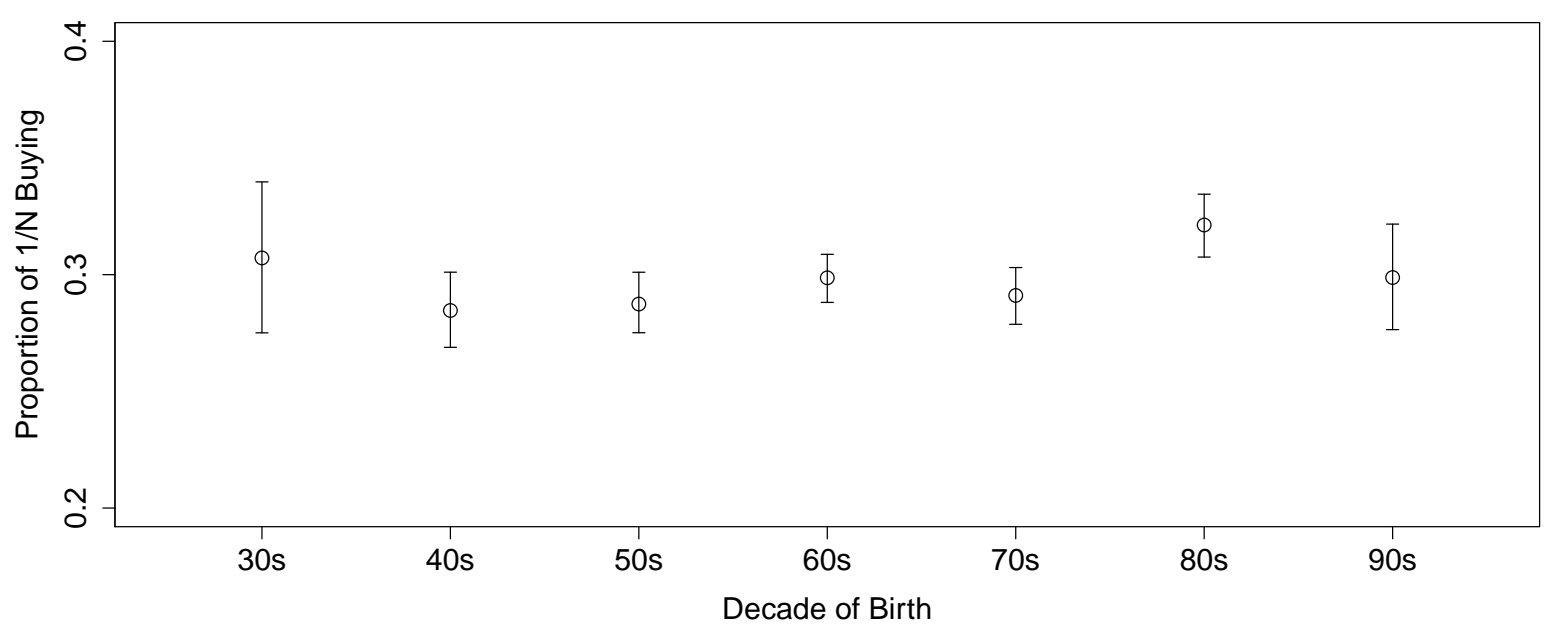

Note: Figure shows proportion of all two-stock buy-days on which the buy-day investments are split equally (in pounds) across the two stocks. Equal is defined in the range $49 \%$ to $51 \%$. Sample is restricted to two-stock buy-days. See Section 2 for details on the sample construction. 95\% confidence intervals illustrated in error bars. 
Figure 3: Naïve Buying Diversification by Portfolio Value and Investment Amount Two-Stock Buy-Days

(A) Account Tenure

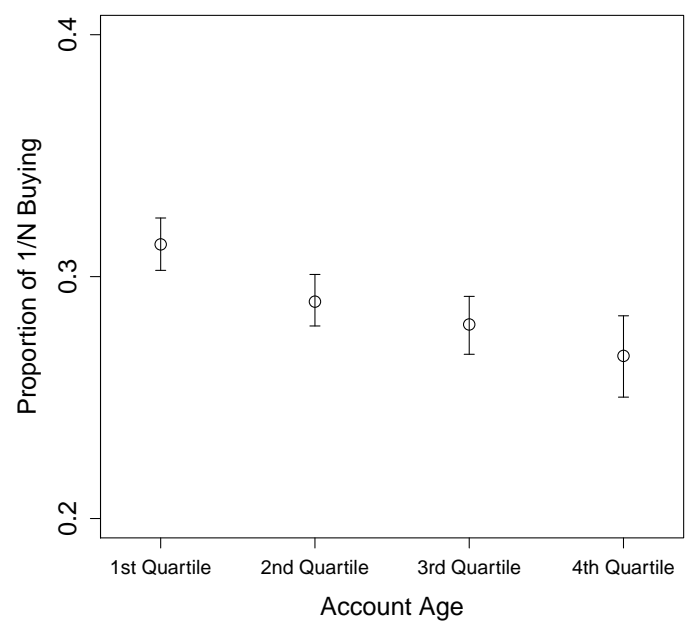

(C) Past 3 Month Returns

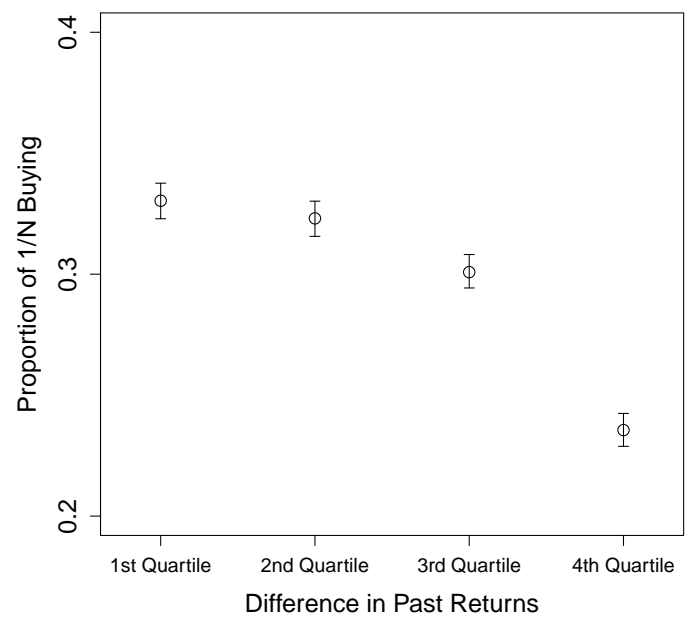

(E) Portfolio Value

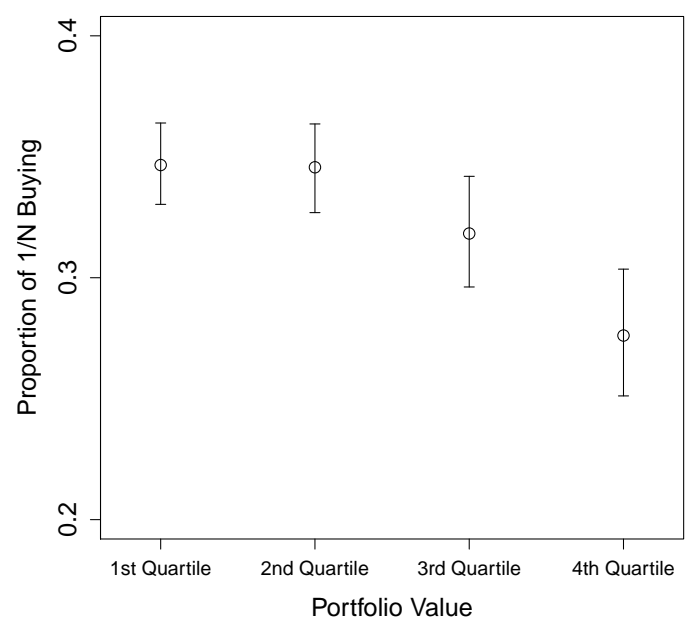

(B) Trading Frequency

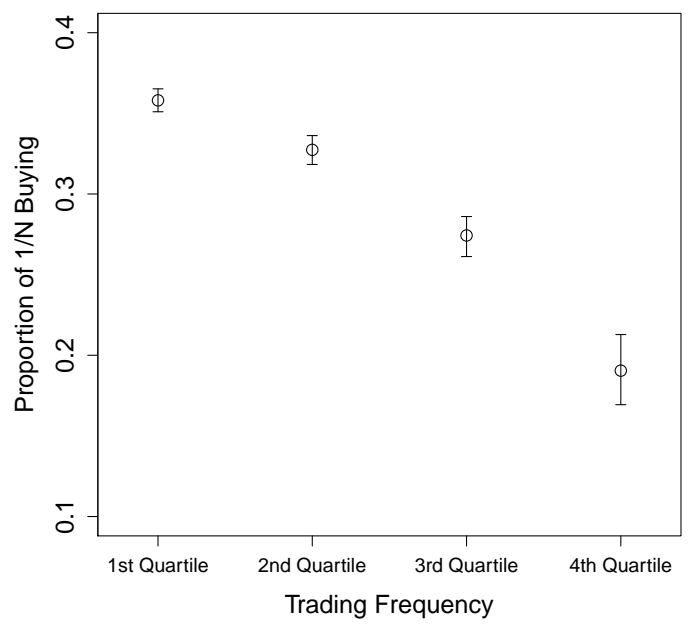

(D) Next 3 Month Returns

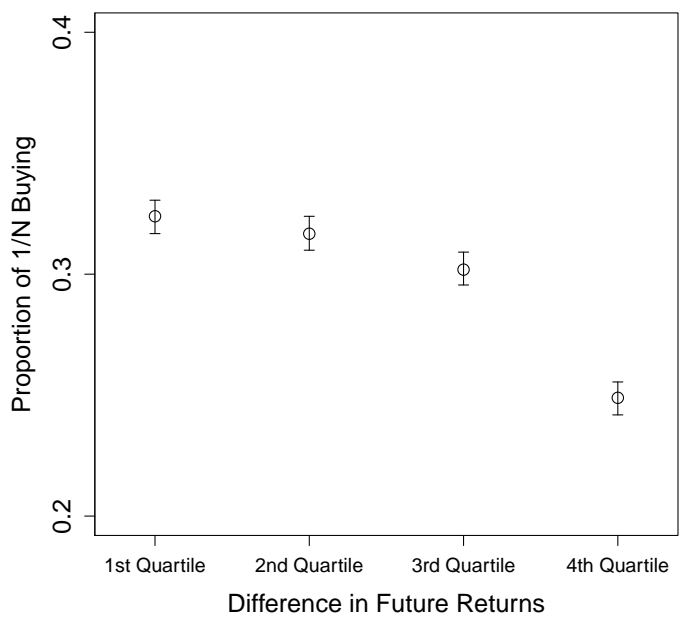

(F) Investment Amount on the Day

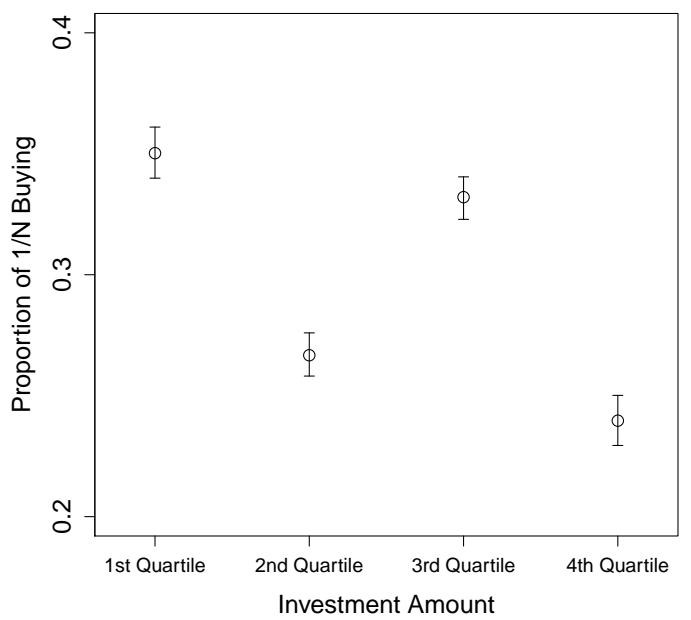

Note: Figure shows proportion of all two-stock buy-days on which the buy-day investments are split equally (in pounds) across the two stocks. Equal is defined in the range $49 \%$ to $51 \%$. Sample is restricted to two-stock buy-days. For Panel E, sample is further restricted to two-stock buy-days in the new accounts data. See Section 2 for details on the samgł construction. $95 \%$ confidence intervals illustrated in error bars. 
Figure 4: Naïve Buying Diversification by Existing Positions and Sales Two-Stock Buy-Days

(A) Existing Position Held

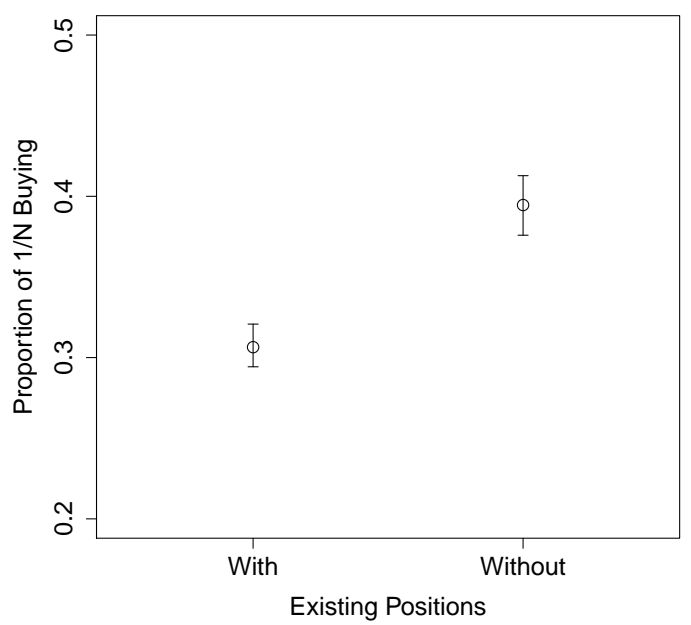

(B) Sale on the Day

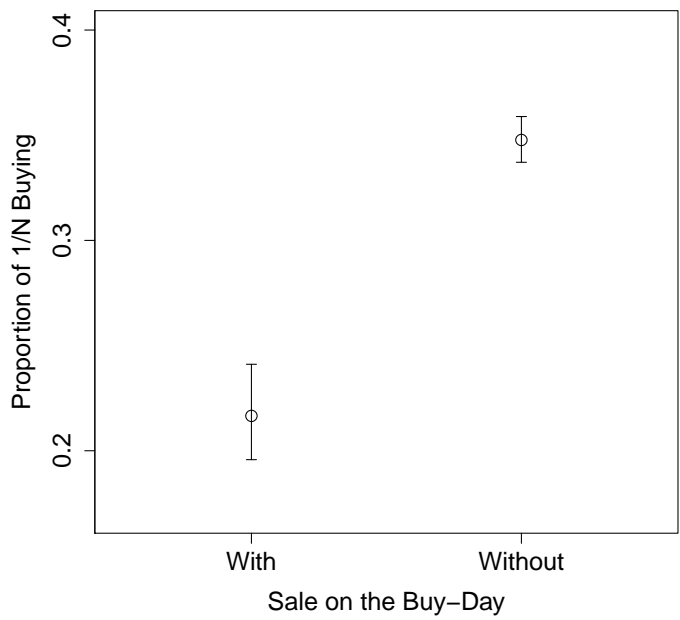

(C) Number of Stocks Held

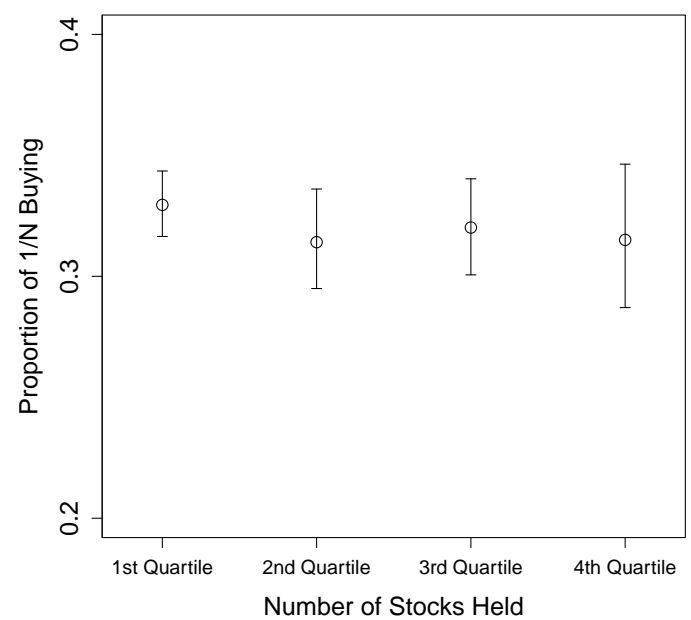

Note: Figure shows proportion of all two-stock buy-days on which the buy-day investments are split equally (in pounds) across the two stocks. Equal is defined in the range $49 \%$ to $51 \%$. Sample is restricted to two-stock buy-days in the new accounts data. See Section 2 for details on the sample construction. $95 \%$ confidence intervals illustrated in error bars. 
Figure 5: Naïve Buying Diversification vs. Naïve Portfolio Diversification Investors Topping-Up Two-Stock Portfolios

(A) Whole Sample
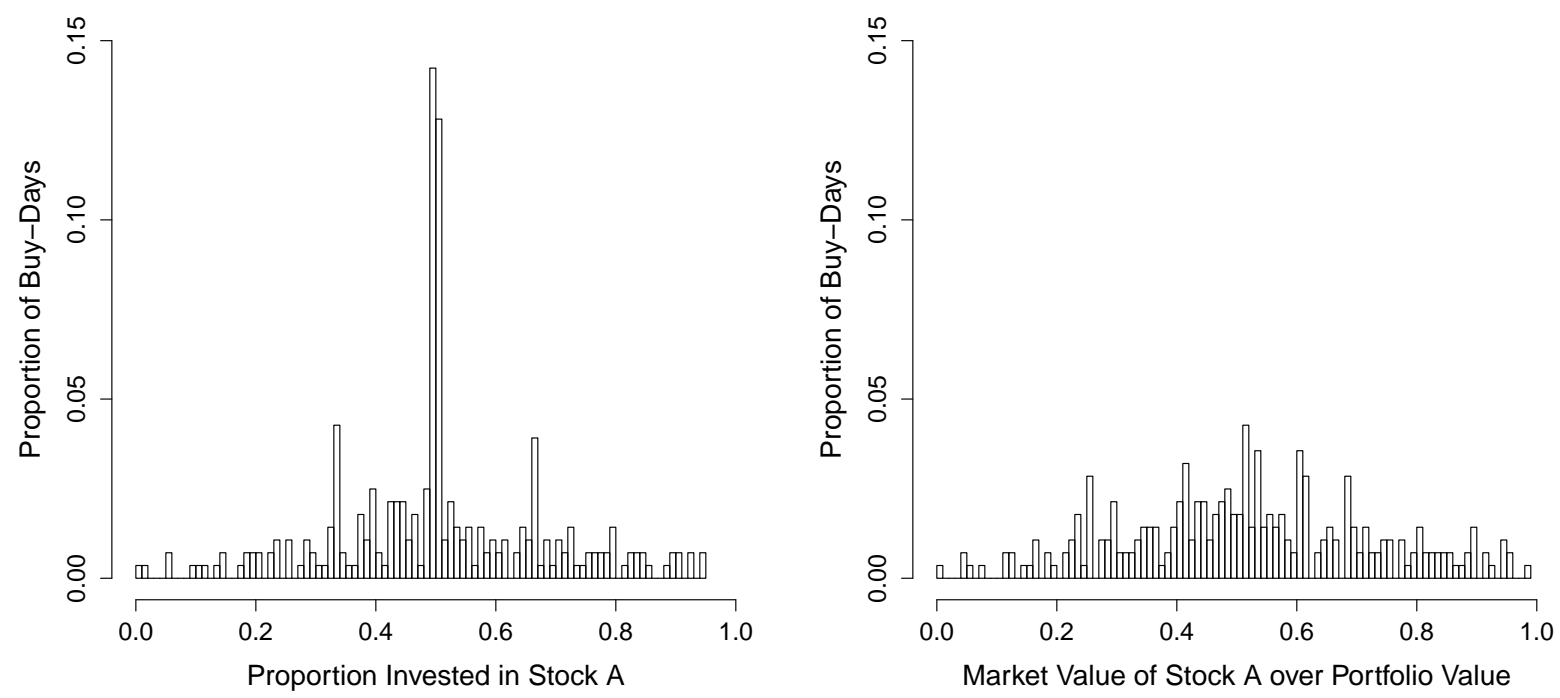

(B) Restricted Sample
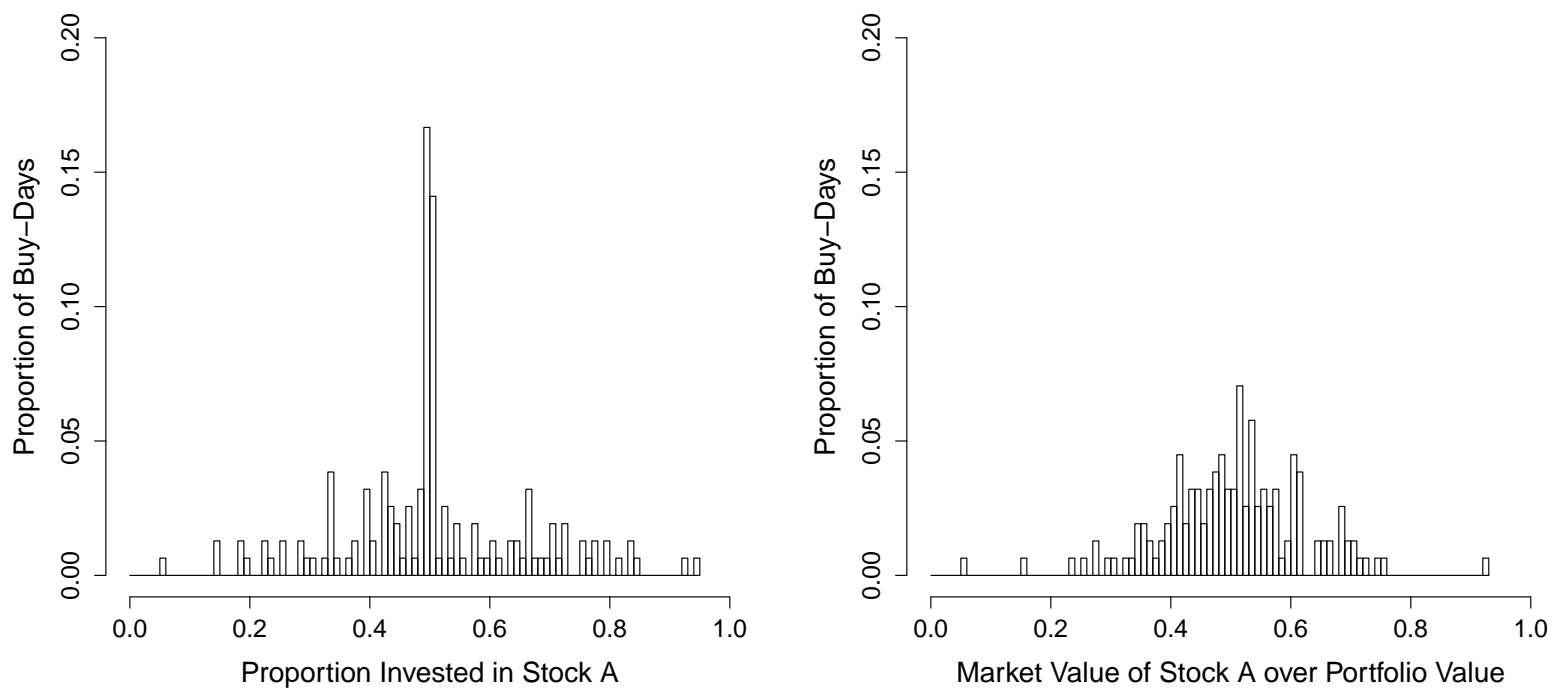

Note: Panel A figure shows a histogram of the proportion of the total buy-day investment (in pounds) invested in Stock A, where Stock A is a randomly chosen stock from the pair of stocks purchased. Bin width is 0.01 . Panel B shows a histogram of the proportion of the end of day investment in the portfolio that is allocated to Stock A, where Stock A is a randomly chosen stock from the pair of stocks purchased. Sample is restricted to two-stock buy-days in the sample of new accounts. See Section 2 for details on the sample construction. Bin width is 0.01 . 
Figure 6: Naïve Buying Diversification vs. Naïve Portfolio Diversification All Buy-Days

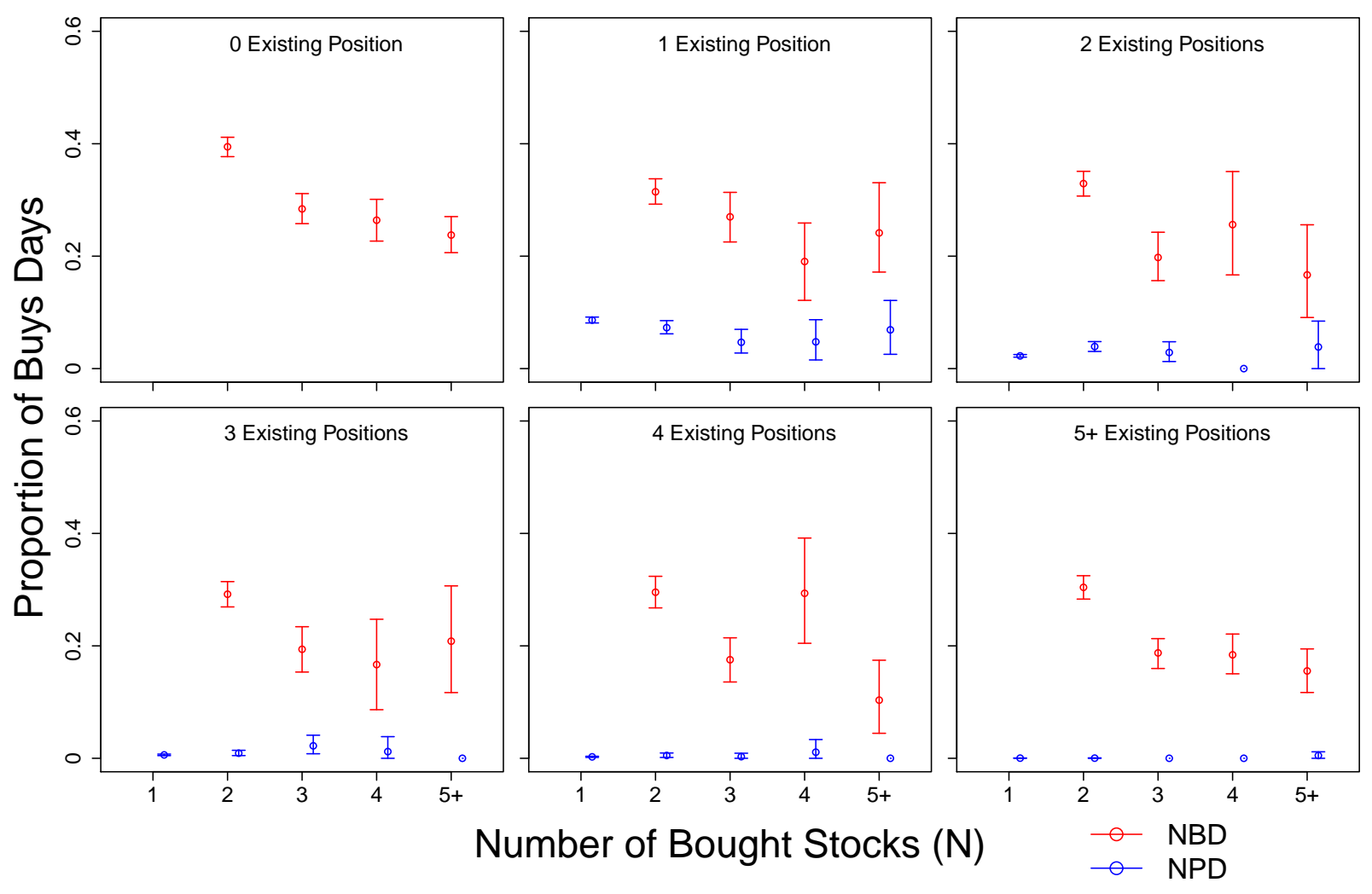

Note: Plots illustrate the proportion of buy-days on which multiple-stock purchases as split across stocks $1 / N$ (in red) and on which the end of day portfolio positions are split $1 / N$ (in blue). Separate panels for number of existing positions within the portfolio at the start of the day, with number of stocks purchased on the day shown on the $\mathrm{x}$-axis of each panel. Sample is restricted to multiple-stock buy-days in the new accounts data. See Section 2 for details on the sample construction. 
Figure 7: Distribution of Total Buy-Day Investment (in $£$ ) by Number of Stocks Bought

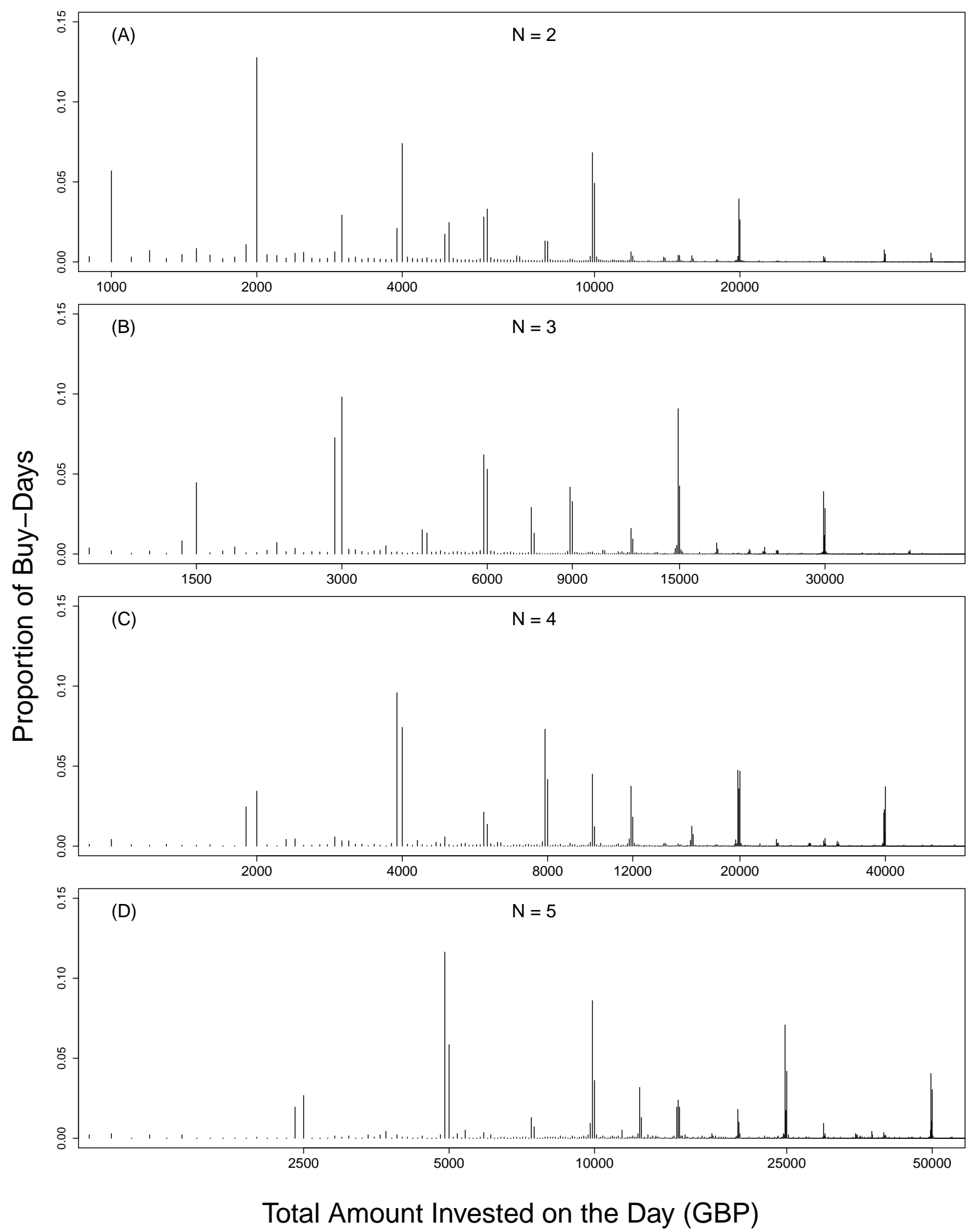

Note: Panels illustrate the distribution of monies invested on the buy-day (in pounds) for multiple-stock buy-days involving $2-5$ stocks. Sample is restricted to multiple-stock buy-days. See Section 2 for details on the sample construction. 
Table 1: 1/N Allocations on Multiple-Stock Buy-Days

(a) Panel A: $(£ P / N \times(1 \pm 0.02))$

\begin{tabular}{ccccc}
\hline$N$ & $1 / N$ Buying $(\%)$ & LL & UL & Buy-days \\
\hline 2 & 29.7 & 29.1 & 30.3 & 177193 \\
3 & 20.3 & 19.4 & 21.1 & 48896 \\
4 & 18.6 & 17.6 & 19.7 & 17672 \\
5 & 17.5 & 16.0 & 19.0 & 7925 \\
$6+$ & 15.2 & 13.1 & 17.3 & 9899 \\
All & 26.3 & 25.6 & 26.9 & 261585 \\
\hline
\end{tabular}

(b) Panel B: $(£ P / N \times(1 \pm 0.05))$

\begin{tabular}{ccccc}
\hline$N$ & $1 / N$ Buying (\%) & LL & UL & Buy-days \\
\hline 2 & 36.5 & 36.0 & 37.1 & 177193 \\
3 & 23.3 & 22.4 & 24.2 & 48896 \\
4 & 20.9 & 19.7 & 22.1 & 17672 \\
5 & 20.1 & 18.6 & 21.7 & 7925 \\
$6+$ & 18.0 & 15.3 & 20.6 & 9899 \\
All & 31.8 & 31.1 & 32.5 & 261585 \\
\hline
\end{tabular}

(c) Panel C: $(£ P / N \times(1 \pm 0.05))$

\begin{tabular}{ccccc}
\hline$N$ & $1 / N$ Buying $(\%)$ & LL & UL & Buy-days \\
\hline 2 & 45.6 & 45.1 & 46.2 & 177193 \\
3 & 27.8 & 26.8 & 28.7 & 48896 \\
4 & 23.9 & 22.6 & 25.2 & 17672 \\
5 & 22.4 & 20.7 & 24.0 & 7925 \\
$6+$ & 20.0 & 17.2 & 22.8 & 9899 \\
All & 39.1 & 38.4 & 39.9 & 261585 \\
\hline
\end{tabular}

Note: Table shows summary data for multiple-stock buy-days. Each row reports the percentage of buy-days involving $N$ stocks in which the proportion invested in each stock falls within the $1 / N$ range, for differing ranges. See Section 3 for details. Lower limit and upper limit values of $95 \%$ confidence intervals from bootstrap mean estimates are reported in LL and UL columns. Sample is restricted to multiple-stock buy-days. See Section 2 for details on the sample construction. 
Table 2: Probit Regression Marginal Effects: $1 / N$ Buying on Two-Stock Buy-Days

\begin{tabular}{|c|c|c|c|c|c|}
\hline & Model 1 & Model 2 & & Model 3 & \\
\hline \multirow[t]{2}{*}{ (intercept) } & -0.085 & -0.076 & & 0.061 & \\
\hline & {$[-0.311,0.119]$} & {$[-0.289,0.129]$} & & {$[-0.159,0.262]$} & \\
\hline \multirow[t]{2}{*}{ Decade of Birth 1930s } & -0.071 & -0.062 & & -0.079 & \\
\hline & {$[-0.280,0.168]$} & {$[-0.278,0.145]$} & & {$[-0.291,0.153]$} & \\
\hline \multirow[t]{2}{*}{ Decade of Birth 1940s } & -0.120 & -0.095 & & -0.102 & \\
\hline & {$[-0.318,0.112]$} & {$[-0.289,0.112]$} & & {$[-0.305,0.110]$} & \\
\hline \multirow[t]{2}{*}{ Decade of Birth 1950s } & -0.088 & -0.062 & & -0.073 & \\
\hline & {$[-0.291,0.140]$} & {$[-0.260,0.145]$} & & {$[-0.273,0.132]$} & \\
\hline \multirow[t]{2}{*}{ Decade of Birth 1960s } & -0.088 & -0.051 & & -0.062 & \\
\hline & {$[-0.288,0.140]$} & {$[-0.251,0.153]$} & & {$[-0.251,0.147]$} & \\
\hline \multirow[t]{2}{*}{ Decade of Birth 1970s } & -0.086 & -0.061 & & -0.065 & \\
\hline & {$[-0.288,0.139]$} & {$[-0.264,0.150]$} & & {$[-0.255,0.137]$} & \\
\hline \multirow[t]{2}{*}{ Decade of Birth 1980s } & -0.092 & -0.067 & & -0.070 & \\
\hline & {$[-0.293,0.133]$} & {$[-0.263,0.133]$} & & {$[-0.265,0.143]$} & \\
\hline \multirow[t]{2}{*}{ Decade of Birth 1990s } & -0.077 & -0.072 & & -0.078 & \\
\hline & {$[-0.280,0.151]$} & {$[-0.280,0.136]$} & & {$[-0.276,0.128]$} & \\
\hline \multirow[t]{2}{*}{ Decade of Birth 2000s } & -0.128 & -0.003 & & -0.024 & \\
\hline & {$[-0.349,0.148]$} & {$[-0.263,0.231]$} & & {$[-0.257,0.206]$} & \\
\hline \multirow[t]{2}{*}{ Male } & -0.009 & 0.004 & & 0.005 & \\
\hline & {$[-0.040,0.026]$} & {$[-0.029,0.035]$} & & {$[-0.027,0.038]$} & \\
\hline \multirow[t]{2}{*}{ Account Tenure 2nd Quartile } & & 0.000 & & 0.010 & \\
\hline & & {$[-0.029,0.031]$} & & {$[-0.026,0.042]$} & \\
\hline \multirow[t]{2}{*}{ Account Tenure 3rd Quartile } & & -0.022 & & -0.010 & \\
\hline & & {$[-0.052,0.009]$} & & {$[-0.041,0.025]$} & \\
\hline \multirow[t]{2}{*}{ Account Tenure 4th Quartile } & & -0.031 & & -0.017 & \\
\hline & & {$[-0.064,0.004]$} & & {$[-0.052,0.020]$} & \\
\hline \multirow[t]{2}{*}{ Ave Num of Trades Per Month } & & -0.006 & ** & -0.004 & $* *$ \\
\hline & & {$[-0.009,-0.004]$} & & {$[-0.008,-0.003]$} & \\
\hline \multirow[t]{2}{*}{ Portfolio Value (x 10000) } & & & & -0.001 & \\
\hline & & & & {$[-0.003,0.000]$} & \\
\hline \multirow[t]{2}{*}{ Num of Stocks in the Portfolio } & & & & 0.004 & $*$ \\
\hline & & & & {$[0.000,0.006]$} & \\
\hline \multirow[t]{2}{*}{ Inv Amount on the Day (x 10000) } & & & & 0.002 & \\
\hline & & & & {$[-0.003,0.008]$} & \\
\hline \multirow[t]{2}{*}{ N (Num of Bought Stocks) } & & & & -0.034 & $* * *$ \\
\hline & & & & {$[-0.047,-0.023]$} & \\
\hline \multirow[t]{2}{*}{ Range in Past 60-Days Returns } & & & & -0.005 & \\
\hline & & & & {$[-0.024,0.001]$} & \\
\hline \multirow[t]{2}{*}{ Range in Next 60-Days Returns } & & & & -0.083 & $* *$ \\
\hline & & & & {$[-0.138,-0.035]$} & \\
\hline \multirow[t]{2}{*}{ Existing Position Dummy } & & & & -0.054 & $* * *$ \\
\hline & & & & {$[-0.084,-0.024]$} & \\
\hline \multirow[t]{2}{*}{ Same-Day Sale Dummy } & & & & -0.084 & $* * *$ \\
\hline & & & & {$[-0.116,-0.053]$} & \\
\hline Log-Likelihood & -14439.59 & -13838.38 & & -13560.05 & \\
\hline
\end{tabular}

Note: Table reports marginal effects from probit regression model estimates. Dependent variable is a 1/0 dummy indicating whether the buy-day investment falls within the $1 / N$ range, defined as $£ P / N \times(1 \pm 0.02)$. Covariates are account characteristics. Day-of-week and Month-of-year dummies included in the model but not shown. Sample is restricted to multiple-stock buy-days in the new accounts data. See Section 2 for details on the sample construction. * denotes statistical significance at the $5 \%$ level, ${ }^{* *}$ at the $1 \%$ level and ${ }^{* * *}$ at the $0.1 \%$ level. The standard errors were corrected for clustering by accounts and buy-days. 
Table 3: Starting and Ending Portfolio Positions on Multiple-Stock Buy-Days

\begin{tabular}{|c|c|c|c|c|c|}
\hline $1 / N$ Existing Positions & $1 / N$ Buying & $1 / N$ Resulting Positions & Proportion of Buy-Days & $\mathrm{LL}$ & UL \\
\hline Yes & Yes & Yes & 0.5 & 0.4 & 0.7 \\
\hline Yes & Yes & No & 0.6 & 0.4 & 0.7 \\
\hline Yes & No & Yes & 0.0 & 0.0 & 0.0 \\
\hline Yes & No & No & 1.1 & 0.9 & 1.3 \\
\hline No & Yes & Yes & 0.1 & 0.0 & 0.1 \\
\hline No & Yes & No & 28.5 & 27.3 & 29.8 \\
\hline No & No & Yes & 0.1 & 0.0 & 0.1 \\
\hline No & No & No & 69.2 & 67.8 & 70.4 \\
\hline
\end{tabular}

Panel (B) $(£ P / N \times(1 \pm 0.05))$

\begin{tabular}{cccccc}
\hline $1 / N$ Existing Positions & $1 / N$ Buying & $1 / N$ Resulting Positions & Proportion of Buy-Days & LL & UL \\
\hline Yes & Yes & Yes & 1.5 & 1.3 & 1.7 \\
Yes & Yes & No & 0.1 & 1.1 \\
Yes & No & Yes & 1.5 \\
Yes & No & No & 1.9 & 0.0 & 0.1 \\
No & Yes & Yes & 32.7 & 1.6 & 2.1 \\
No & Yes & No & 0.2 & 0.1 & 0.2 \\
No & No & Yes & 62.2 & 34.5 \\
No & No & No & 6.1 & 0.3 \\
\hline
\end{tabular}

Panel $(\mathrm{C})(£ P / N \times(1 \pm 0.1))$

\begin{tabular}{|c|c|c|c|c|c|}
\hline $1 / N$ Existing Positions & $1 / N$ Buying & $1 / N$ Resulting Positions & Proportion of Buy-Days & LL & UL \\
\hline Yes & Yes & Yes & 2.7 & 2.4 & 3.1 \\
\hline Yes & Yes & No & 2.2 & 2.0 & 2.5 \\
\hline Yes & No & Yes & 0.2 & 0.1 & 0.2 \\
\hline Yes & No & No & 2.7 & 2.4 & 3.0 \\
\hline No & Yes & Yes & 0.2 & 0.2 & 0.3 \\
\hline No & Yes & No & 37.2 & 36.0 & 38.3 \\
\hline No & No & Yes & 0.3 & 0.2 & 0.4 \\
\hline No & No & No & 54.5 & 53.3 & 55.8 \\
\hline
\end{tabular}

Note: Table shows summary data for multiple-stock buy days. Each row reports the percentage of buy-days by combinations of existing positions at the beginning of the day, buying split, and resulting positions in the $1 / N$ range, for differing ranges. Lower limit and upper limit values of $95 \%$ confidence intervals from bootstrap mean estimate reported in LL and UL columns. Sample is restricted to multiple-stock buy-days in the new accounts data. See Section 2 for details on the sample construction. 
Table 4: Naïve Portfolio Diversification, Restricted Sample Buy-Days

\begin{tabular}{lcccc}
\hline$N$ & $1 / N$ Portfolios (\%) & LL & UL & Buy-days \\
\hline 2 & 3.5 & 2.9 & 4.3 & 2622 \\
3 & 1.4 & 1.0 & 1.9 & 2549 \\
4 & 0.8 & 0.3 & 1.3 & 1052 \\
5 & 1.1 & 0.2 & 2.3 & 467 \\
$6+$ & 0.0 & 0.0 & 0.0 & 482 \\
All & 2.0 & 1.6 & 2.3 & 7172 \\
\hline
\end{tabular}

Note: Table shows summary data for multiplestock buy days. Each row reports the percentage of buy-days resulting positions in the $1 / N$ range, for different number of stocks in the portfolio. Lower limit and upper limit values of $95 \%$ confidence intervals from bootstrap mean estimate reported in LL and UL columns. Sample is restricted to multiple-stock buy-days in the new accounts data. See Section 2 for details on the sample construction. 
Table 5: Naïve Portfolio Diversification By N Stocks Purchased and N Positions All Buy-Days

\begin{tabular}{|c|c|c|c|c|c|c|}
\hline & Single Stock Purchase & 2 Stock Purchase & 3 Stock Purchase & 4 Stock Purchase & 5 Sstock Purchase & $6+$ Sstock Purchase \\
\hline \multirow[t]{2}{*}{0 Existing Position } & NA & 36.5 & 24.9 & 22.4 & 25.0 & 14.1 \\
\hline & & {$[34.8,38.3]$} & {$[22.0,27.5]$} & {$[19.0,25.8]$} & {$[19.5,30.6]$} & {$[10.6,17.8]$} \\
\hline \multirow[t]{2}{*}{1 Existing Position } & 8.6 & 7.3 & 4.7 & 4.8 & 10.0 & 4.5 \\
\hline & {$[8.1,9.2]$} & {$[6.0,8.5]$} & $2.6,7.1]$ & {$[1.6,8.7]$} & {$[2.3,18.9]$} & {$[0.0,10.5]$} \\
\hline \multirow[t]{2}{*}{2 Existing Positions } & 2.3 & 3.9 & 2.9 & 0.0 & 2.8 & 4.8 \\
\hline & {$[2.0,2.5]$} & {$[3.0,5.0]$} & {$[1.2,4.5]$} & {$[0.0,0.0]$} & $0.0,9.7]$ & {$[0.0,11.9]$} \\
\hline \multirow{2}{*}{3 Existing Positions } & 0.6 & 0.9 & 2.2 & 1.2 & 0.0 & 0.0 \\
\hline & {$[0.5,0.7]$} & {$[0.5,1.4]$} & $0.8,4.0]$ & {$[0.0,3.8]$} & $0.0,0.0]$ & {$[0.0,0.0]$} \\
\hline \multirow[t]{2}{*}{4 Existing Positions } & 0.3 & 0.5 & 0.3 & 1.1 & 0.0 & 0.0 \\
\hline & {$[0.2,0.4]$} & {$[0.2,0.9]$} & $0.0,0.9]$ & {$[0.0,3.6]$} & $0.0,0.0]$ & {$[0.0,0.0]$} \\
\hline \multirow[t]{2}{*}{5 Existing Positions } & 0.1 & 0.2 & 0.0 & 0.0 & 8.1 & 0.0 \\
\hline & {$[0.0,0.1]$} & {$[0.0,0.4]$} & {$[0.0,0.0]$} & {$[0.0,0.0]$} & {$[0.0,18.8]$} & {$[0.0,0.0]$} \\
\hline \multirow{2}{*}{$6+$ Existing Positions } & 0.0 & 0.0 & 0.0 & 0.0 & 0.0 & 0.3 \\
\hline & {$[0.0,0.0]$} & {$[0.0,0.0]$} & $0.0,0.0]$ & {$[0.0,0.0]$} & $0.0,0.0]$ & {$[0.0,0.8]$} \\
\hline
\end{tabular}

Note: Table shows data for all buy-days. Each cell reports the percentage of buy-days which end in $1 / N$ allocations by number of existing positions at the start of the buy-day and number stocks purchased on the day. Cell $[0,1]$ empty as it takes a value of $100 \%$ by construction. Values in square brackets report lower limit and upper limit values of $95 \%$ confidence intervals from bootstrap mean estimate. Sample is restricted to multiple-stock buy-days in the new accounts data. See Section 2 for details on the sample construction. The numbers in the first row slightly differ from those in Table 6 due to intraday price movement. 
Table 6: Naïve Buying Diversification By $N$ Stocks Purchased and $N$ Positions All Buy-Days

\begin{tabular}{|c|c|c|c|c|c|}
\hline & 2 Stock Purchase & 3 Stock Purchase & 4 Stock Purchase & 5 Sstock Purchase & $6+$ Sstock Purchase \\
\hline \multirow[t]{2}{*}{0 Existing Position } & 39.5 & 28.4 & 26.4 & 29.9 & 19.4 \\
\hline & {$[37.7,41.4]$} & {$[25.7,31.3]$} & {$[22.8,30.5]$} & {$[24.3,35.5]$} & {$[15.5,23.6]$} \\
\hline \multirow{2}{*}{1 Existing Position } & 31.5 & 27.0 & 19.0 & 30.0 & 19.7 \\
\hline & {$[29.4,33.7]$} & {$[22.6,31.7]$} & {$[12.5,26.6]$} & {$[17.5,42.2]$} & {$[10.3,29.4]$} \\
\hline \multirow[t]{2}{*}{2 Existing Positions } & 32.9 & 19.8 & 25.6 & 19.4 & 14.3 \\
\hline & {$[30.6,35.0]$} & {$[15.9,24.1]$} & {$[16.5,34.9]$} & $7.1,34.1]$ & {$[4.8,25.6]$} \\
\hline \multirow[t]{2}{*}{3 Existing Positions } & 29.2 & 19.4 & 16.7 & 20.6 & 21.1 \\
\hline & {$[26.9,31.6]$} & {$[15.4,23.5]$} & {$[9.0,24.4]$} & $8.3,35.0]$ & {$[8.3,34.1]$} \\
\hline \multirow{2}{*}{4 Existing Positions } & 29.5 & 17.5 & 29.3 & 12.2 & 7.9 \\
\hline & {$[26.7,32.4]$} & {$[13.6,21.8]$} & {$[20.8,38.9]$} & {$[4.2,23.4]$} & {$[0.0,17.6]$} \\
\hline \multirow[t]{2}{*}{5 Existing Positions } & 30.6 & 17.8 & 20.9 & 35.1 & 17.2 \\
\hline & {$[27.6,33.8]$} & {$[13.4,22.5]$} & {$[13.3,29.5]$} & {$[16.7,53.1]$} & {$[5.3,33.3]$} \\
\hline \multirow[t]{2}{*}{$6+$ Existing Positions } & 30.4 & 18.9 & 18.0 & 15.2 & 13.9 \\
\hline & {$[28.2,32.6]$} & {$[16.1,21.6]$} & {$[14.4,21.7]$} & {$[11.4,19.4]$} & {$[9.2,19.1]$} \\
\hline
\end{tabular}

Note: Table shows data for all buy-days. Each cell reports the percentage of buy-days on which the investor splits the buy-day investment $1 / N$, by number of existing positions at the start of the buy-day and number stocks purchased on the day. Values in square brackets report lower limit and upper limit values of $95 \%$ confidence intervals from bootstrap mean estimate.Sample is restricted to multiple-stock buy-days in the new accounts data. The numbers in the first row slightly differ from those in Table 5 due to intraday price movement. 
Table 7: Sharpe Ratio Simulations for NPD vs. NBD

Panel A: Low Idiosyncratic Risk

\begin{tabular}{ccccccc}
\hline \multirow{2}{*}{ Strategy } & \multicolumn{2}{c}{$N_{i}=5$} & \multicolumn{2}{c}{$N_{i}=10$} & \multicolumn{2}{c}{$N_{i}=20$} \\
& $T=120$ & $T=360$ & $T=120$ & $T=360$ & $T=120$ & $T=360$ \\
\hline NPD & 0.127 & 0.128 & 0.136 & 0.139 & 0.134 & 0.140 \\
NBD & 0.125 & 0.124 & 0.135 & 0.135 & 0.133 & 0.136 \\
& $(0.661)$ & $(0.111)$ & $(0.736)$ & $(0.161)$ & $(0.791)$ & $(0.151)$ \\
\hline
\end{tabular}

Panel B: High Idiosyncratic Risk

\begin{tabular}{ccccccc}
\hline Strategy & \multicolumn{2}{c}{$N_{i}=5$} & \multicolumn{2}{c}{$N_{i}=10$} & \multicolumn{2}{c}{$N_{i}=20$} \\
& $T=120$ & $T=360$ & $T=120$ & $T=360$ & $T=120$ & $T=360$ \\
\hline NPD & 0.091 & 0.091 & 0.109 & 0.107 & 0.126 & 0.119 \\
NBD & 0.081 & 0.079 & 0.097 & 0.084 & 0.116 & 0.092 \\
& $(0.015)$ & $(0.000)$ & $(0.004)$ & $(0.000)$ & $(0.014)$ & $(0.000)$ \\
\hline
\end{tabular}

Note: Table shows Sharpe ratios from simulations based upon a one-factor model. The values in parentheses is the $p$-value for the difference in the Sharpe ratios between NPD and NBD strategies, from a $t$-test. 


\section{ONLINE APPENDIX}

\section{Appendix}

\section{A. Simulation Model}

In this appendix we describe the simulation model presented in Section 6 .

Our interest is in estimating the portfolio performance (here measured by the Sharpe ratio) of NBD vs. NPD. We simulate $T$-month long monthly portfolio values according to the single-factor model used in DeMiguel, Garlappi, and Uppal (2009). A portfolio held by an investor $i$ consists of $N_{i}$ risk assets including a single factor. Annualized excess returns of the factor, $R_{b, t}$, are drawn from the normal distribution $R_{b, t} \sim N\left(\mu_{b}, \sigma_{b}^{2}\right)$. Annualized excess returns of the remaining $N_{i}-1$ risk assets, $R_{a, t}$, follow $R_{a, t}=B R_{b, t}+\epsilon_{t}$, where $B$ is the factor loadings and $\epsilon_{t}$ is a noise drawn from the normal distribution $\epsilon_{t} \sim N\left(0, \Sigma_{t}\right) . \Sigma_{t}$ is assumed to be a diagonal matrix where an average of the squared root of the diagonal elements represents the average idiosyncratic risk across $N_{i}-1$ assets. Annualized risk free returns are drawn from $N\left(\mu_{f}, \sigma_{f}^{2}\right)$, where $\mu_{f}=0.02$ and $\sigma_{f}=0.02$.

In the simulation, an investor rebalances the portfolio and invests additional money at monthly frequency. We assume that the investment amount is held constant across months. At each time step (i.e., each month), NPD investors sell and buy risk assets to equalize their market values while NBD investors allocate the new money equally to $N_{i}$ assets without selling any. For simplicity, we assume that a unit of assets is completely divisible and assume zero trading fees. ${ }^{26}$

From the $T$-month long price paths for $N_{i}$ risk assets, we calculate $T-1$ monthly portfolio returns and a Sharpe ratio for both on NPD and NBD strategies. A simulation is repeated by 1,000 times, resulting in 1,000 Sharpe ratios for each of NPD and NBD strategies. ${ }^{27}$ We then conduct a $t$-test to examine the statistical significance of differences

26 The number of trades per month tends to be larger for NPD investors than for NBD investors. Given this, commissions might tend to be larger for NPD investors.

${ }^{27}$ In DeMiguel, Garlappi, and Uppal (2009), a portfolio return at $t$ is calculated, using investment weights for $N_{i}$ assets which are estimated using asset returns in previous $M$ months. (I.e., investors rebalance a portfolio each month using weights estimated in previous $M$-month window.) They repeat the calculation of a portfolio return from $t=M+1$ to $t=T$, resulting in $T-M$ monthly portfolio returns (rolling-sample 
in Sharpe ratios between the two strategies.

In order to examine the sensitivity of simulation results to the correlation in returns across assets, we conduct simulations in two settings-low and high idiosyncratic risk simulations. The parameter values used in the low idiosyncratic risk simulation are the same as those in DeMiguel, Garlappi, and Uppal (2009): $\mu_{b}=0.08$ and $\sigma_{b}=0.16, B$ are evenly spread from 0.5 to 1.5 , and the squared root of the diagonal elements of $\Sigma_{t}$ (i.e., idiosyncratic volatilities) are drawn from the uniform distribution ranging between 0.10 and 0.30. In the high idiosyncratic risk simulation, the support of the uniform distribution for $\Sigma_{t}$ are shifted to the range between 0.40 and 0.60 while other parameter values unchanged from the low idiosyncratic risk simulation. We conduct simulations for each combination of $N_{i}=\{5,10,20\}$ and $T=\{120,360\}$.

method). A Sharpe ratio is calculated based on those $T-M$ portfolio returns $(T=24,000)$. In our simulation, we do not use the rolling-sample method because we focus on how investors allocate new money over time in addition to an initial investment. 
Figure A1: Number of Stocks Bought on Multiple-Stock Buy-Days

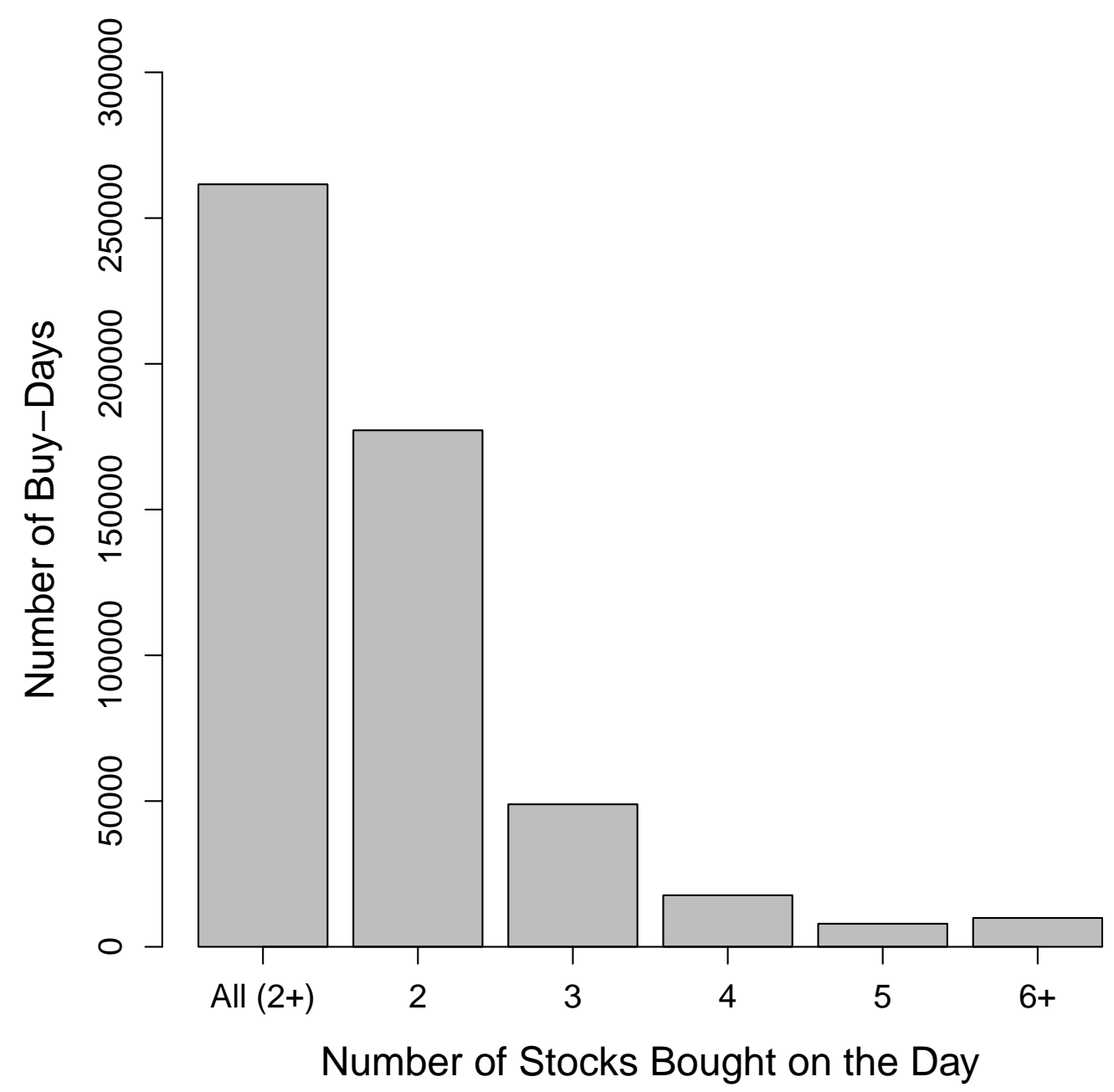

Note: Sample comprises all multiple-stock buy-days. Right-most column includes all multiple-stock buy-days on which 6 or more stocks were purchased. 
Figure A2: Number of Stocks Bought and Market Volatility

(A) Market Volatility and Mean Stocks Purchased

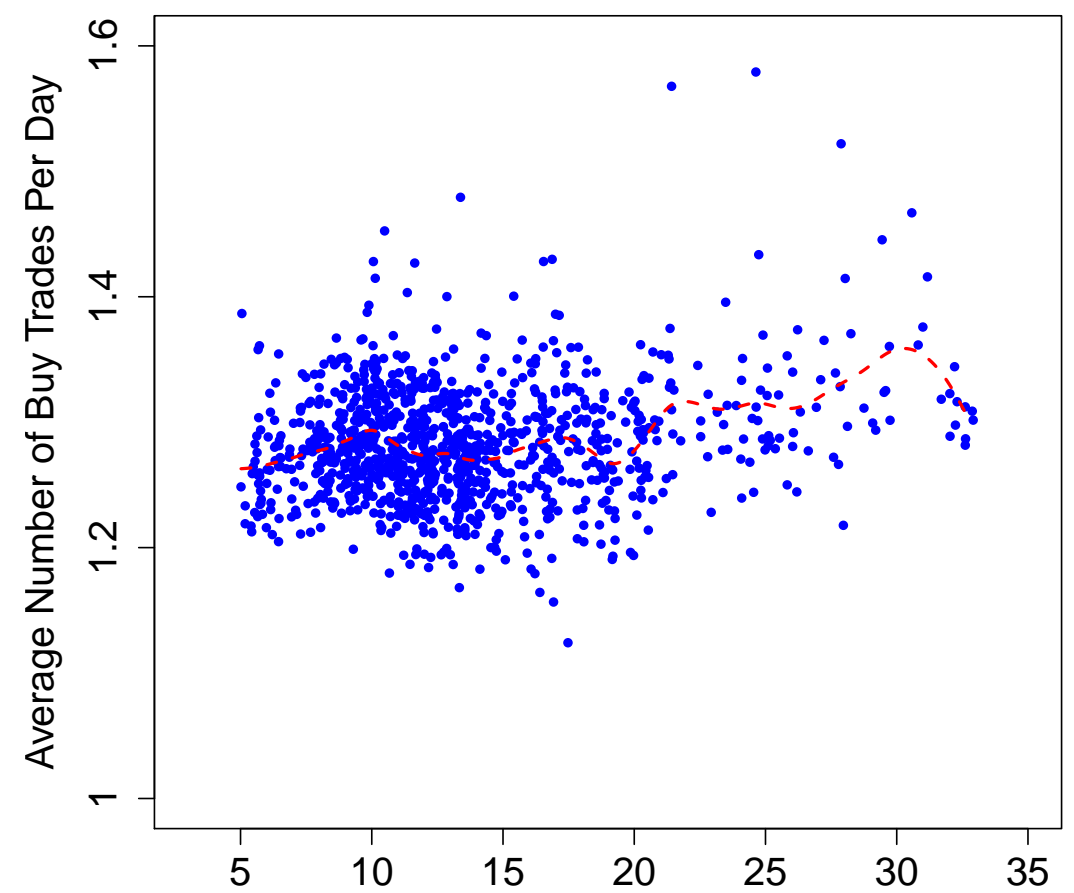

FTSE 100 Volatility (20-days Rolling; Annualized (\%))

(B) Mean Stocks Purchased by Calendar Days

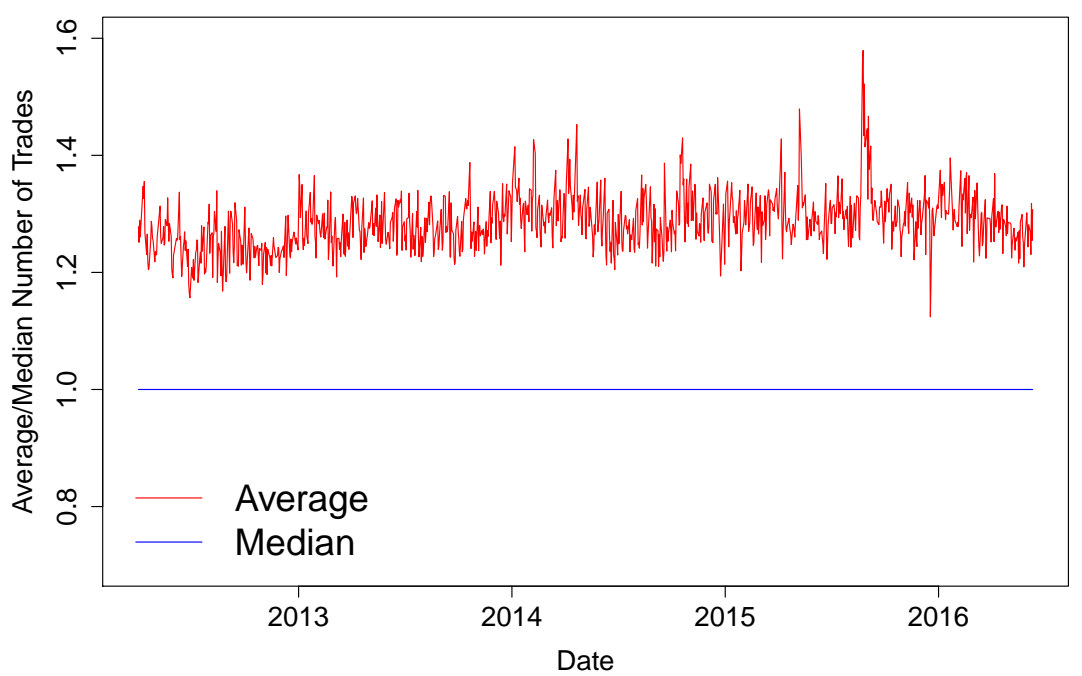

Note: Panel A shows the correlation between the average number of buy trades per day (among accounts making at least one buy trade on the day) and the volatility of the FTSE 100. The dashed red line is a fitted smoothing spline. Panel B shows the average and median number of buy trades per day (among accounts making at least one buy trade on the day) by calendar date of the sample period. The blue lines represents the median value and the red line represents the average value. Sample comprises all buy-days (including both single-stock and multiple-stock buy-days). 
Figure A3: Proportion of Buy-Days with 1/N Buying by Number of Stocks Purchased

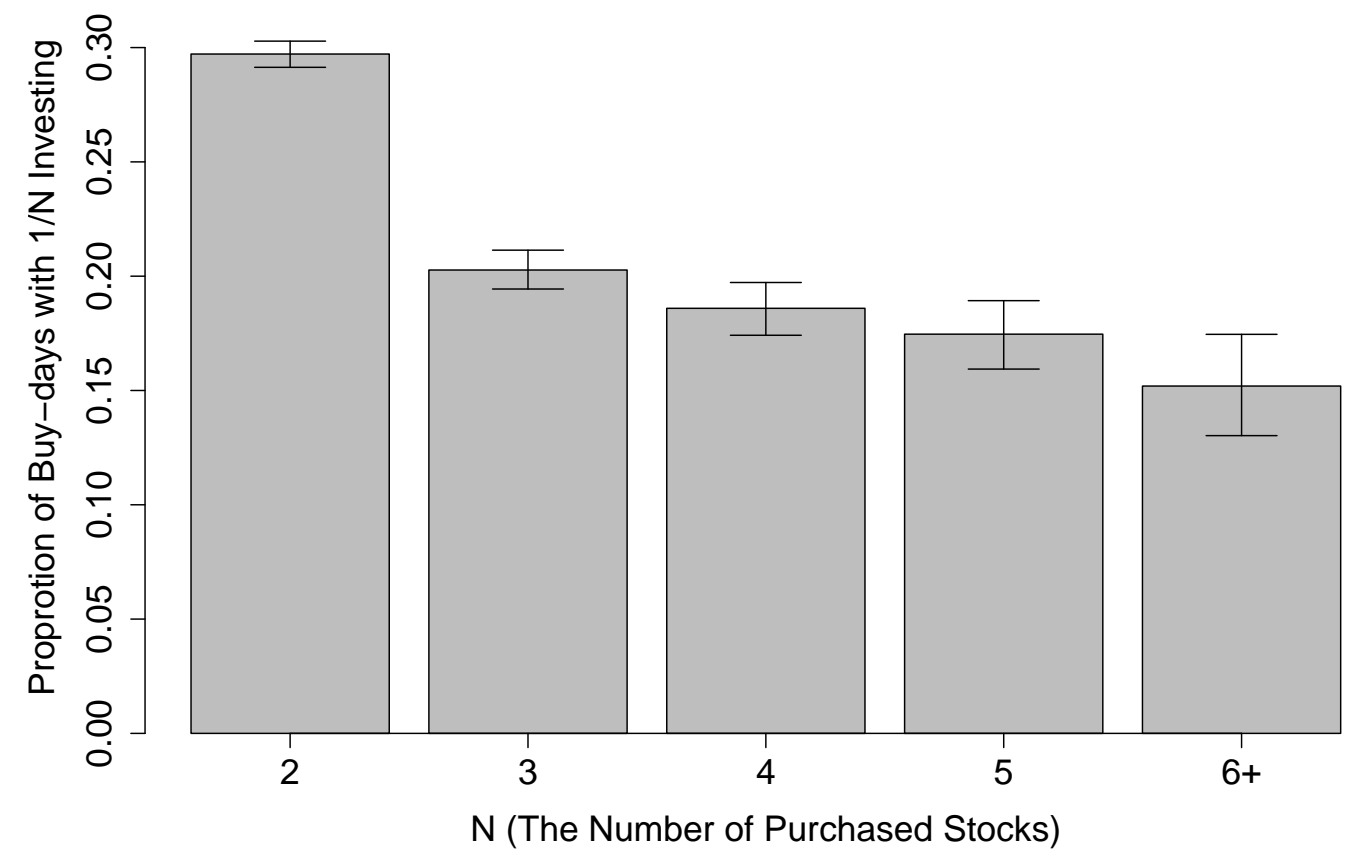

Note: Bar heights illustrate the proportion of all $N$-stock buy-days on which buy-day investments are split equally (in pound values) across the $N$ stocks purchased. Equal is defined in the range $49 \%$ to $51 \%$. Sample comprises all buy-days on which investors buy two or more stocks. $95 \%$ confidence intervals illustrated in error bars. 
Figure A4: $1 / N$ Allocation of Money Amount vs. $1 / N$ Allocation of Number of Shares (A) Allocation of Number of Shares
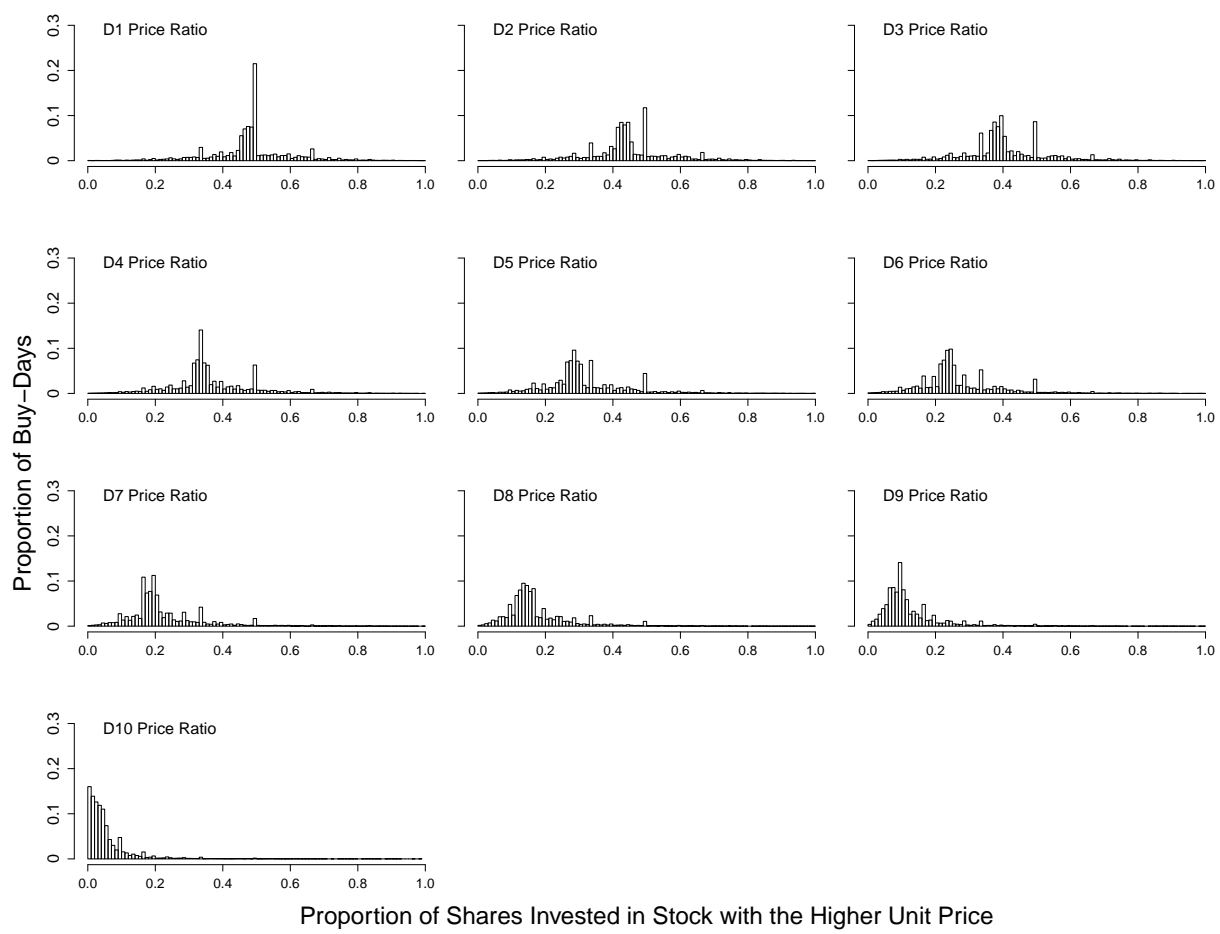

(B) Allocation of Money Amount
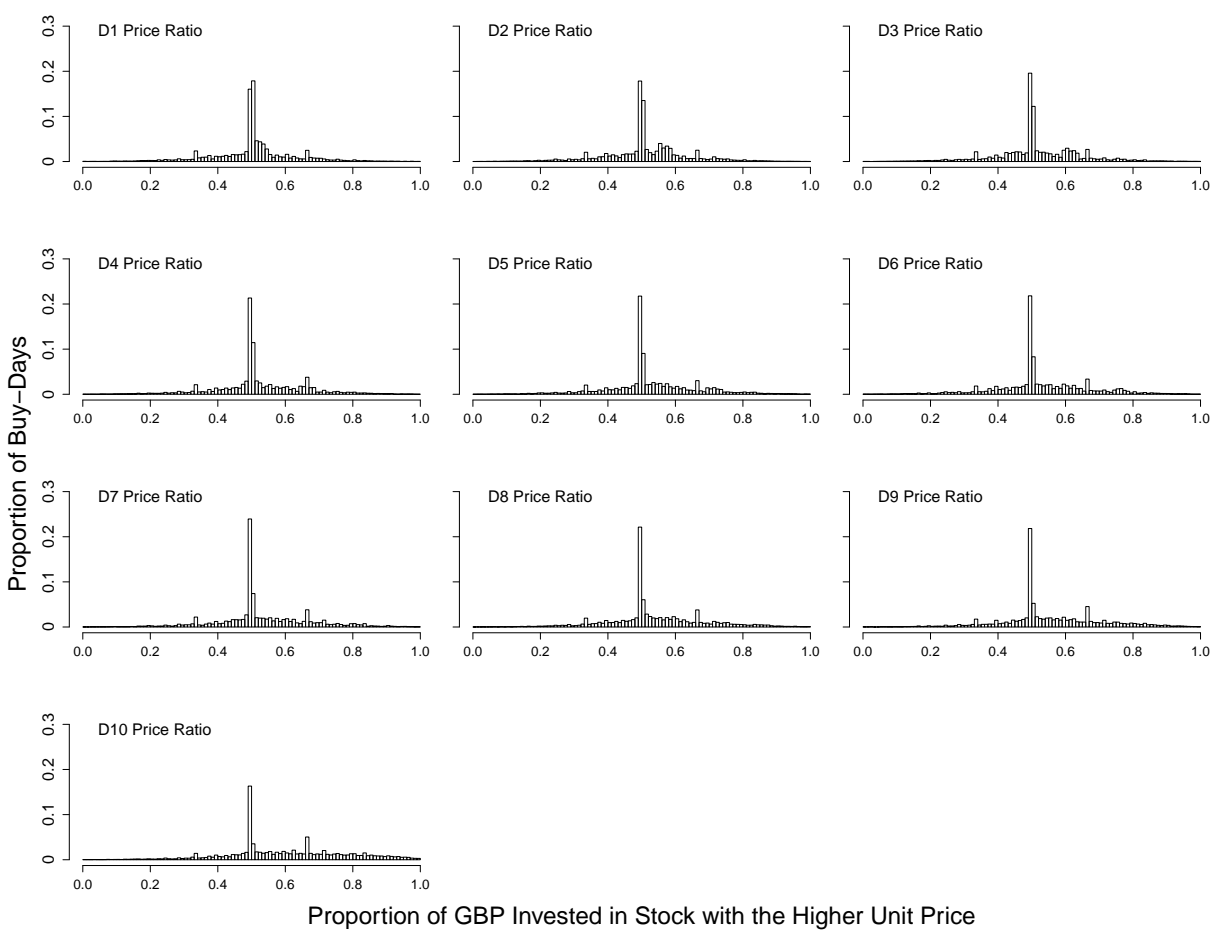

Note: Figure shows allocations of investments on two-stock buy days. Samples for sub-plots are divided into deciles of the ratio of the unit price of shares, where Decile 1 is the lowest ratio (unit price of shares are closest together) and Decile 10 is the highest ratio (unit price of shares are farthest apart). Panel A shows a histogram of the proportion of the total number of shares purchased which is allocated to the higher unit price stock. Panel B shows a histogram of the proportion of the total buy-day investment (in pounds) which is allocated to the higher unit price stock. 
Figure A5: Proportion of Two-Stock Buy-Day Investment in Stock A by Gender

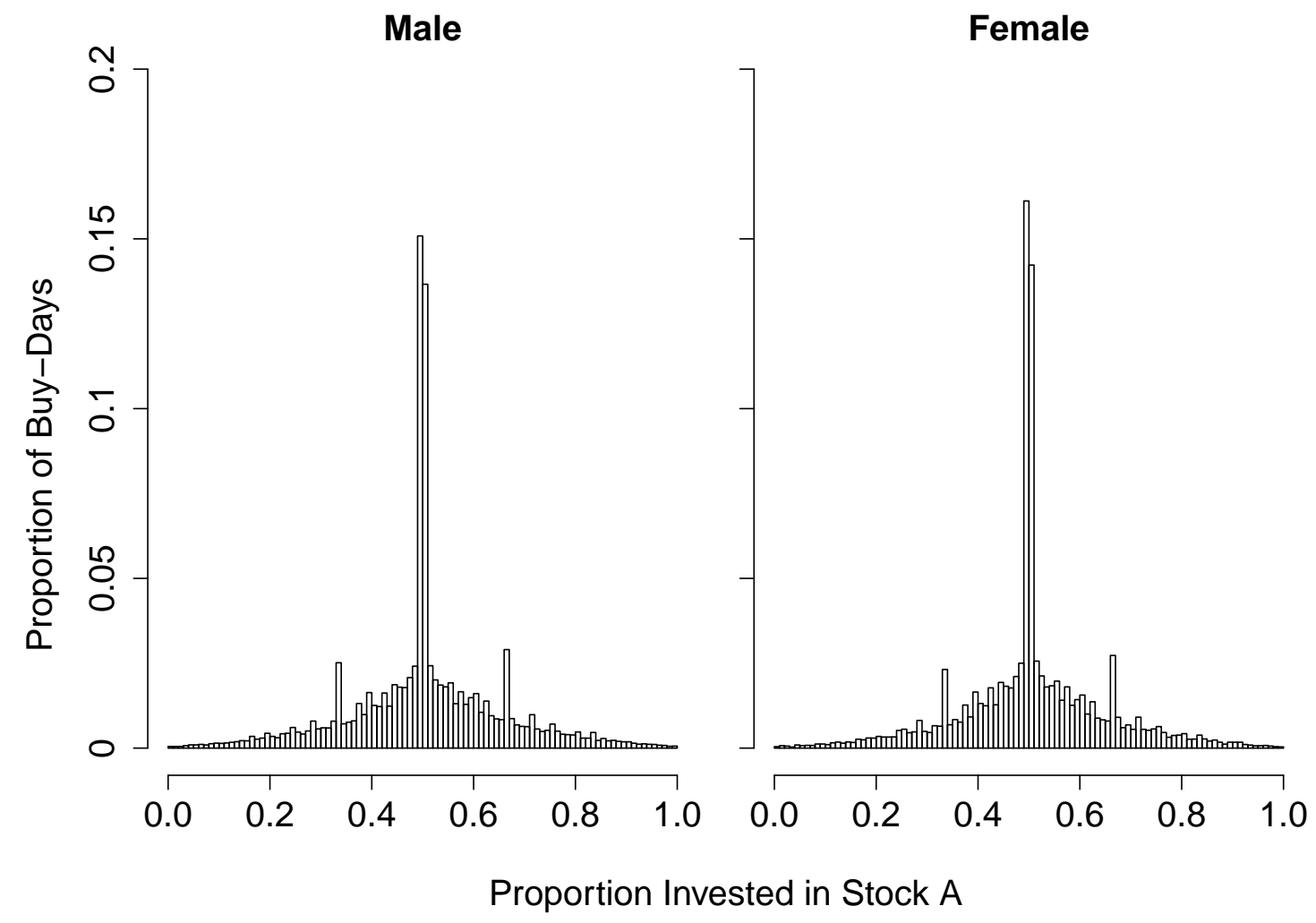

Note: Figures show histograms of the proportion of the total buy-day investment (in pounds) which is invested in Stock A, where Stock A is a randomly chosen stock from the pair of stocks purchased. Sample comprises all buy-days on which investors buy exactly two stocks. Bin width is 0.01 . 
Figure A6: Distribution of Investor Decade of Birth

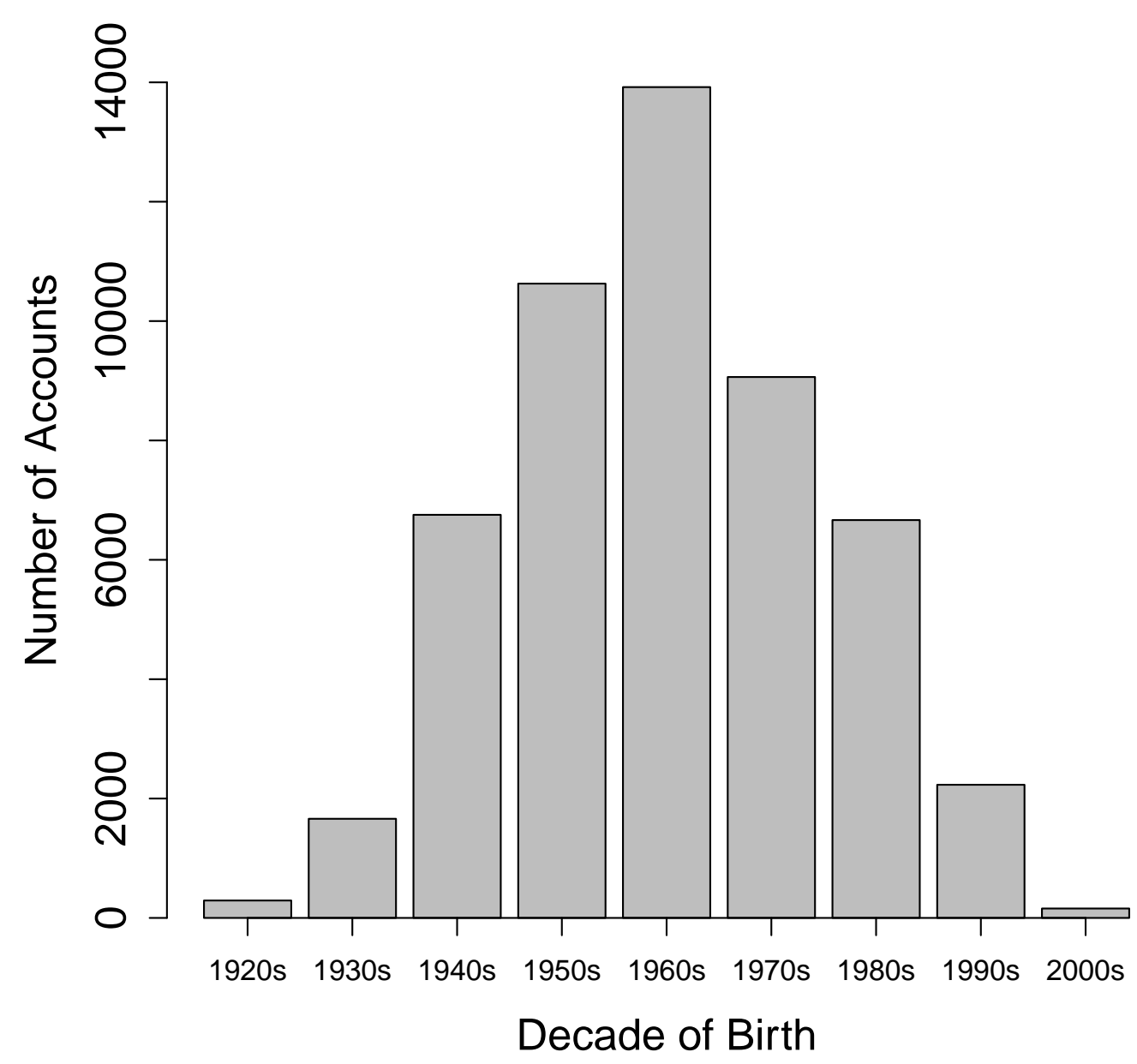

Note: Figure shows a histogram (frequency) of two-stock buy-days by decade of birth of the investor. 
Figure A7: Proportion of Two-Stock Buy-Day Investment in Stock A by Age of Investor

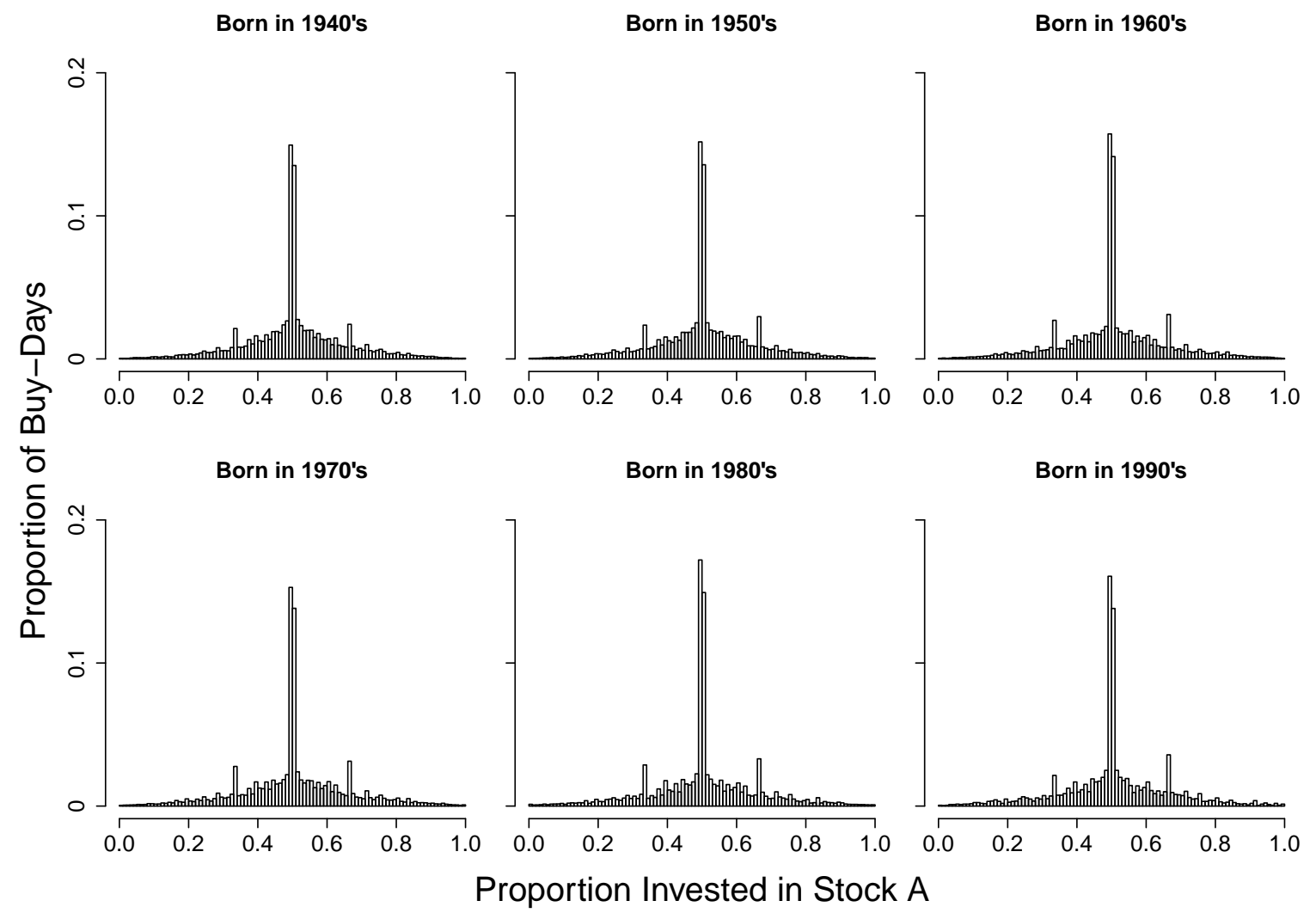

Note: Figures show histograms of the proportion of the total buy-day investment (in pounds) which is invested in Stock A, where Stock A is a randomly chosen stock from the pair of stocks purchased. Sample comprises all buy-days on which investors buy exactly two stocks. Bin width is 0.01 . 
Figure A8: Proportion of Two-Stock Buy-Day Investment in Stock A by Account Tenure

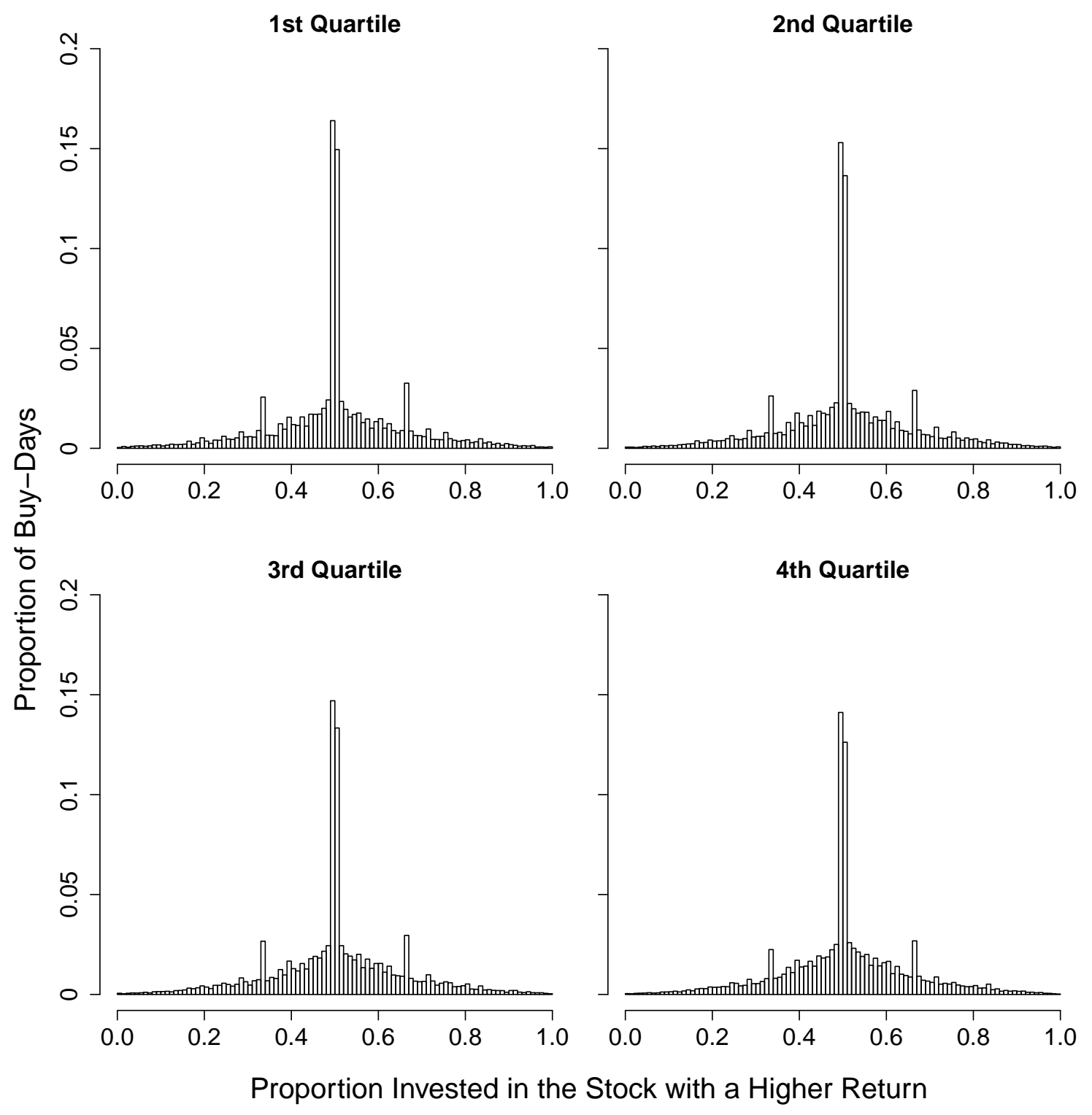

Note: Figures show histograms of the proportion of the total buy-day investment (in pounds) which is invested in Stock A, where Stock A is a randomly chosen stock from the pair of stocks purchased. Sample comprises all buy-days on which investors buy exactly two stocks. Bin width is 0.01 . 
Figure A9: Proportion of Two-Stock Buy-Day Investment in Stock A by Trading Frequency

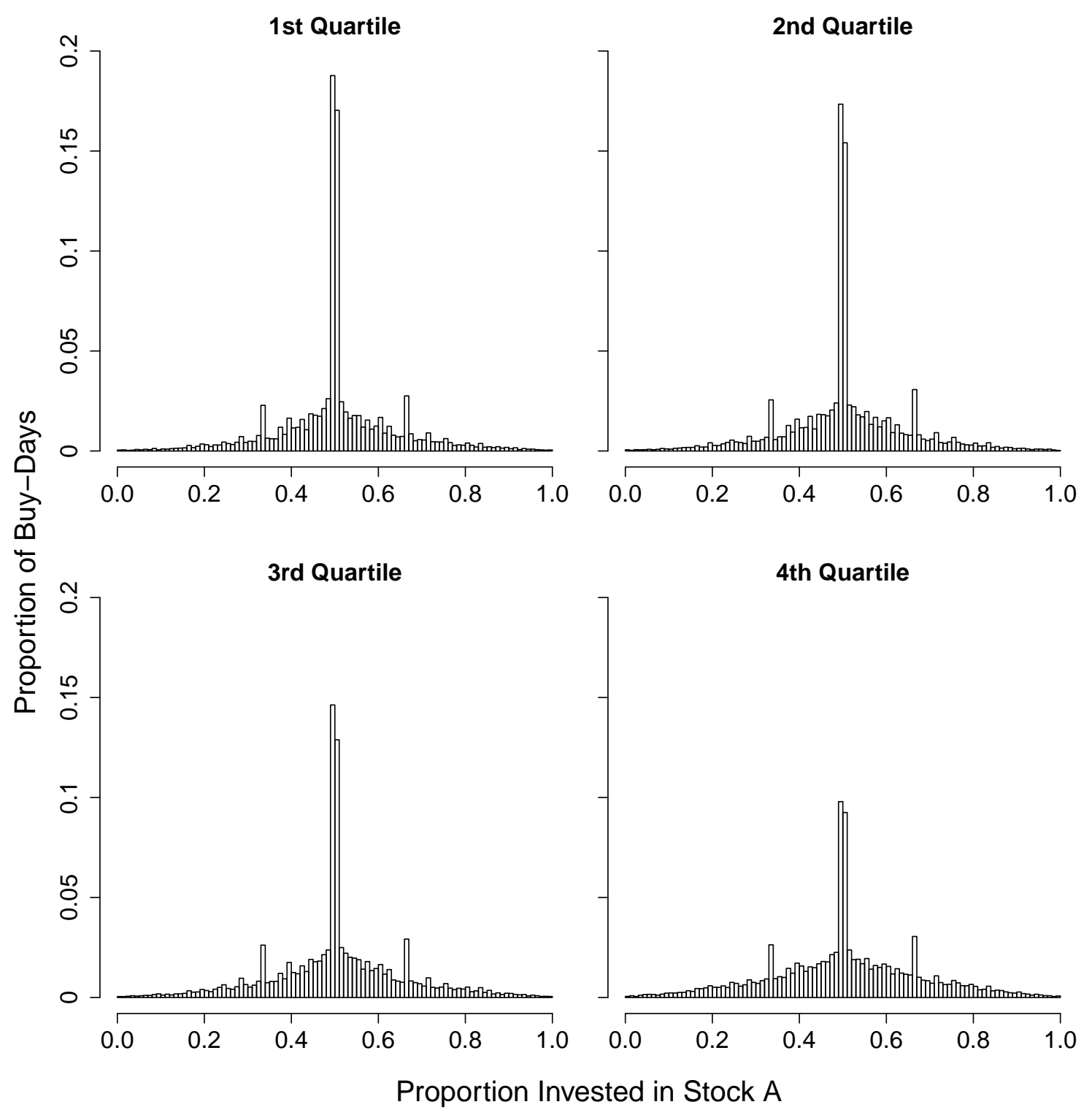

Note: Figures show histograms of the proportion of the total buy-day investment (in pounds) which is invested in Stock A, where Stock A is a randomly chosen stock from the pair of stocks purchased. Sample comprises all buy-days on which investors buy exactly two stocks. Bin width is 0.01 . 
Figure A10: Naïve Buying Diversification by Day and Month Two-Stock Buy-Days

(A) Day of the Week

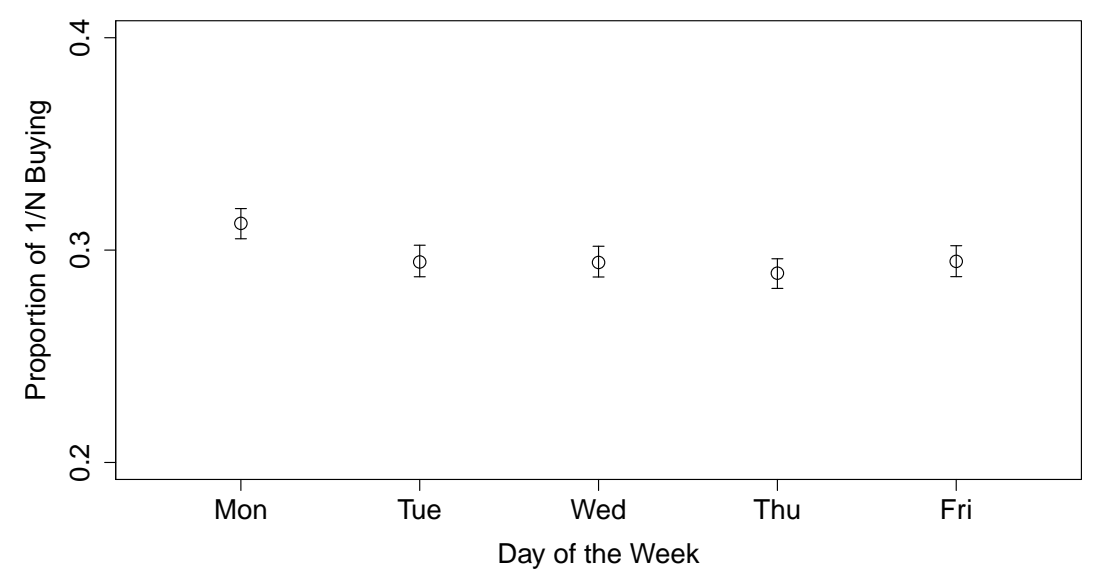

(B) Month of the Year

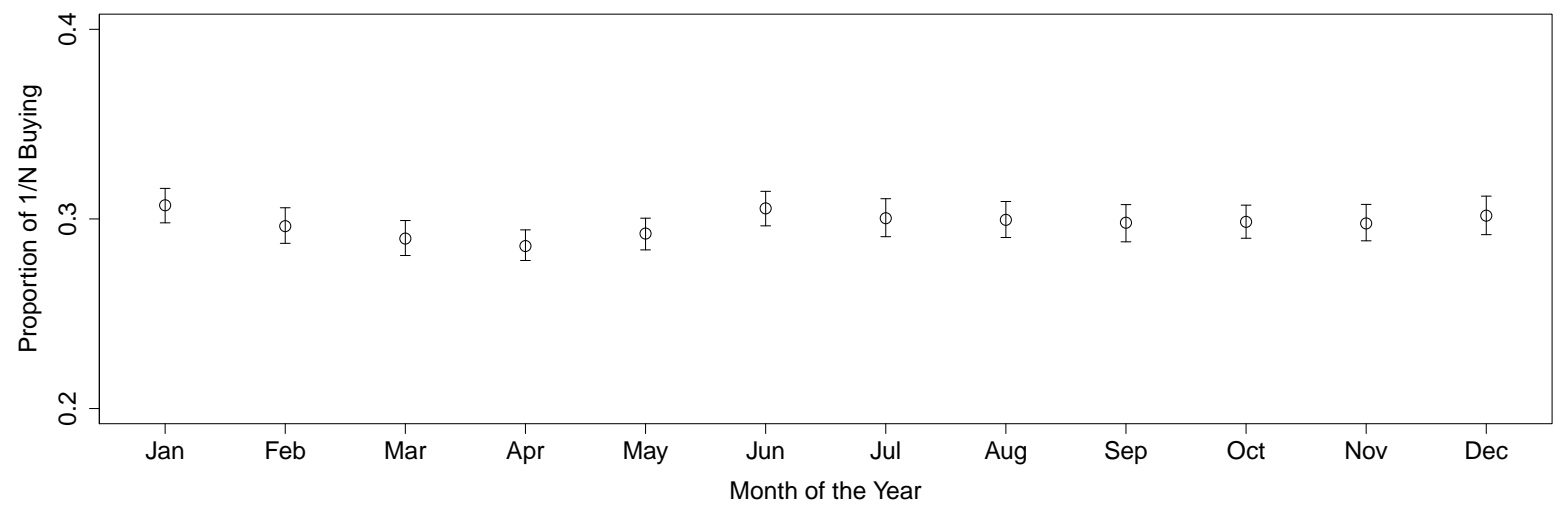

Note: Figure shows proportion of all two-stock buy-days on which the buy-day investments are split equally (in pounds) across the two stocks. Sample is restricted to two-stock buy-days. See Section 2 for details on the sample construction. Equal is defined in the range $49 \%$ to $51 \%$. $95 \%$ confidence intervals illustrated in error bars. 
Figure A11: Distribution of Investment Day of Week

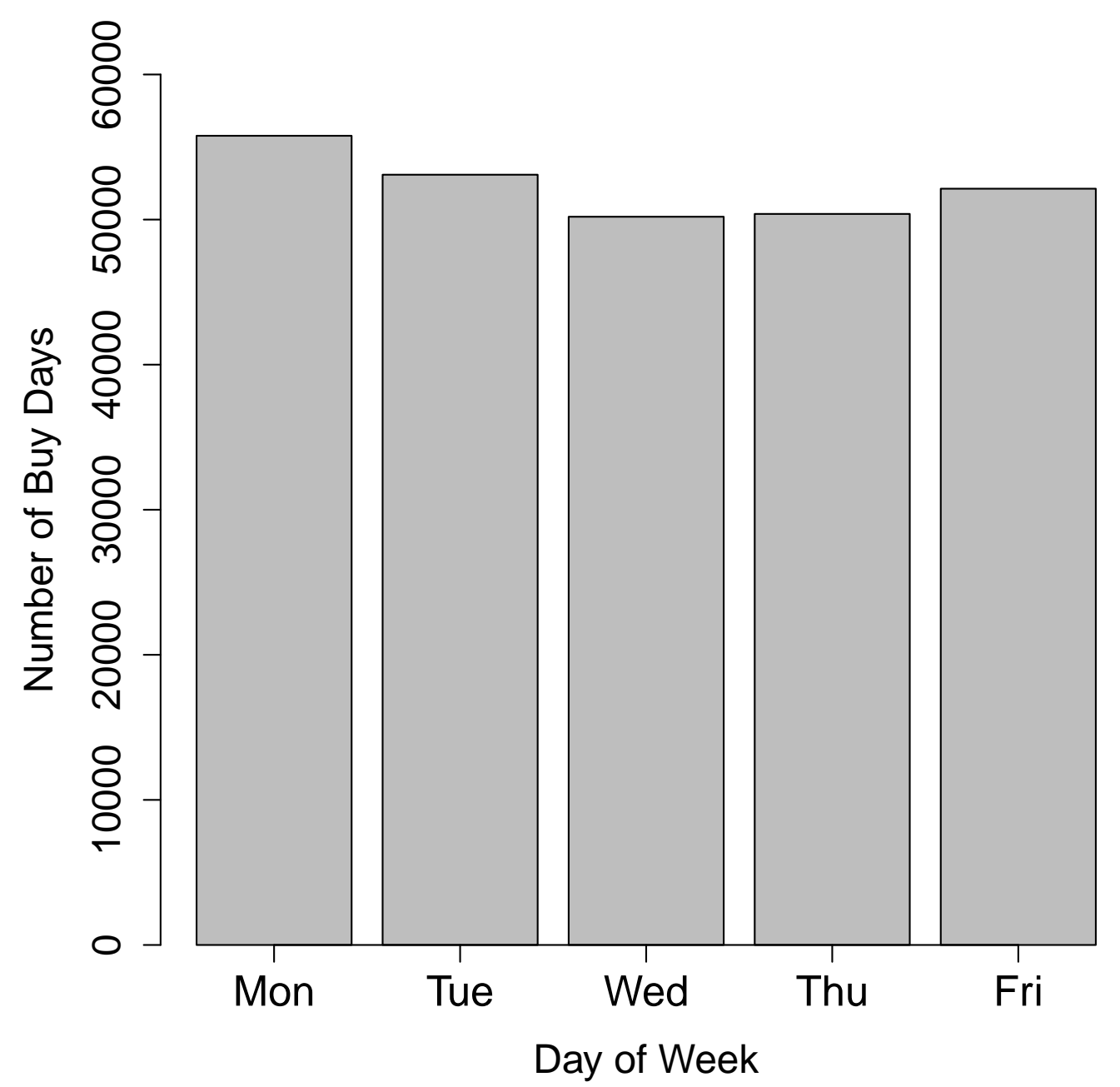

Note: Figure shows a histogram (frequency) of two-stock buy-days by day of the week. 
Figure A12: Proportion of Two-Stock Buy-Day Monies Invested in Stock A by Day

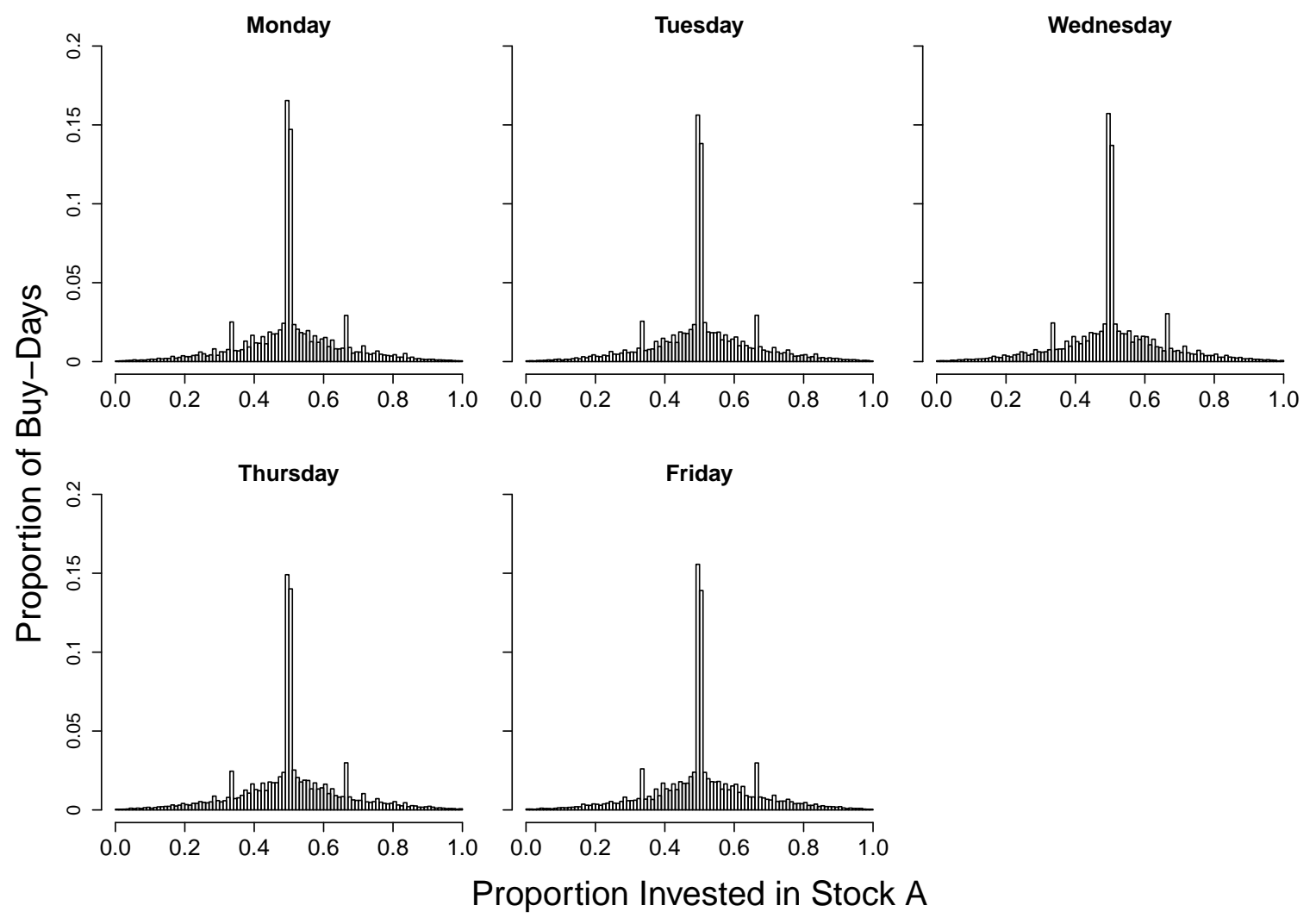

Note: Figures show histograms of the proportion of the total buy-day investment (in pounds) which is invested in Stock A, where Stock A is a randomly chosen stock from the pair of stocks purchased. Sample comprises all buy-days on which investors buy exactly two stocks. Bin width is 0.01 . 
Figure A13: Distribution of Investment Month of Year

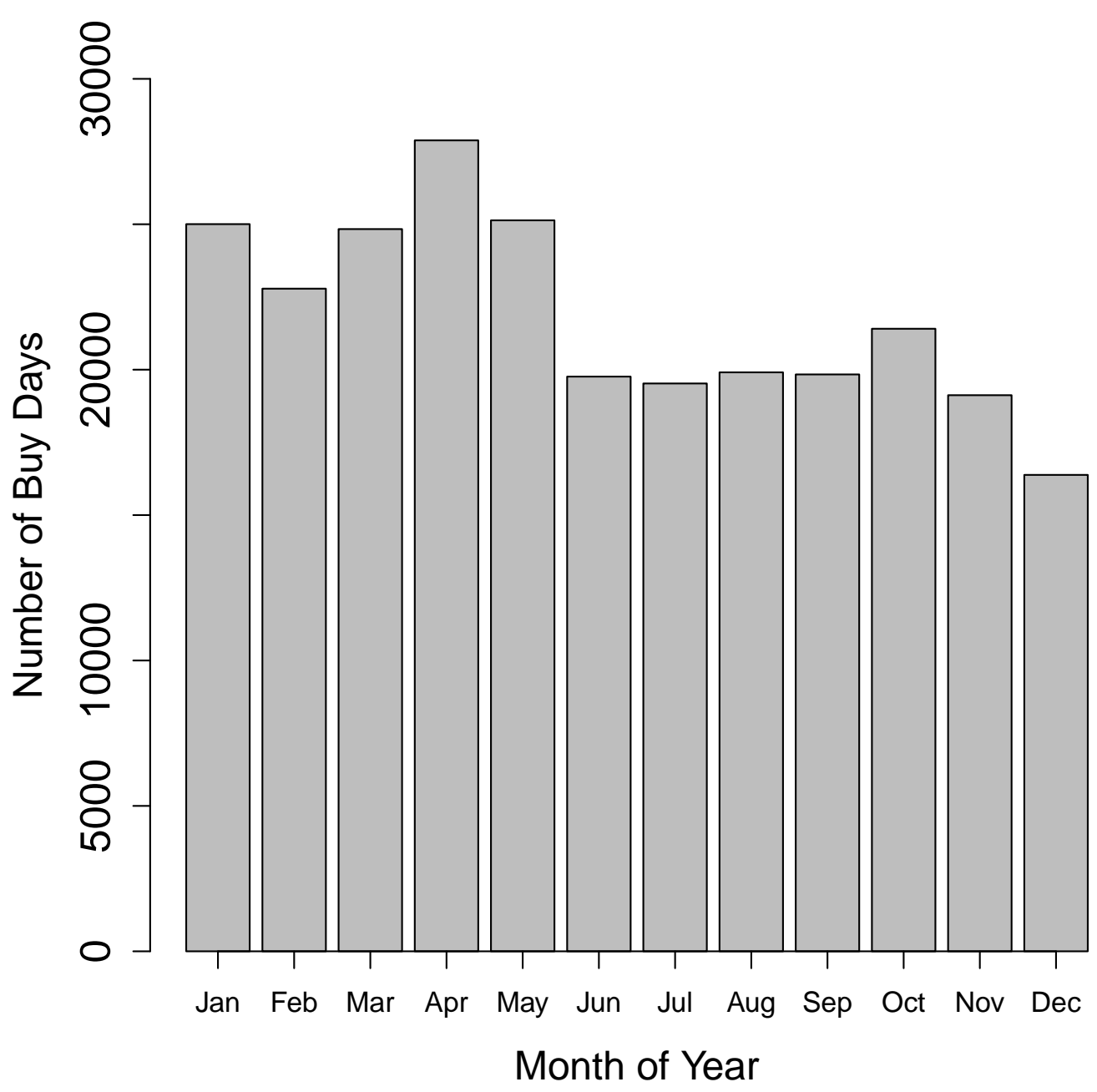

Note: Figure shows a histogram (frequency) of two-stock buy-days by month of the year. 
Figure A14: Proportion of Two-Stock Buy-Day Monies Invested in Stock A by Month
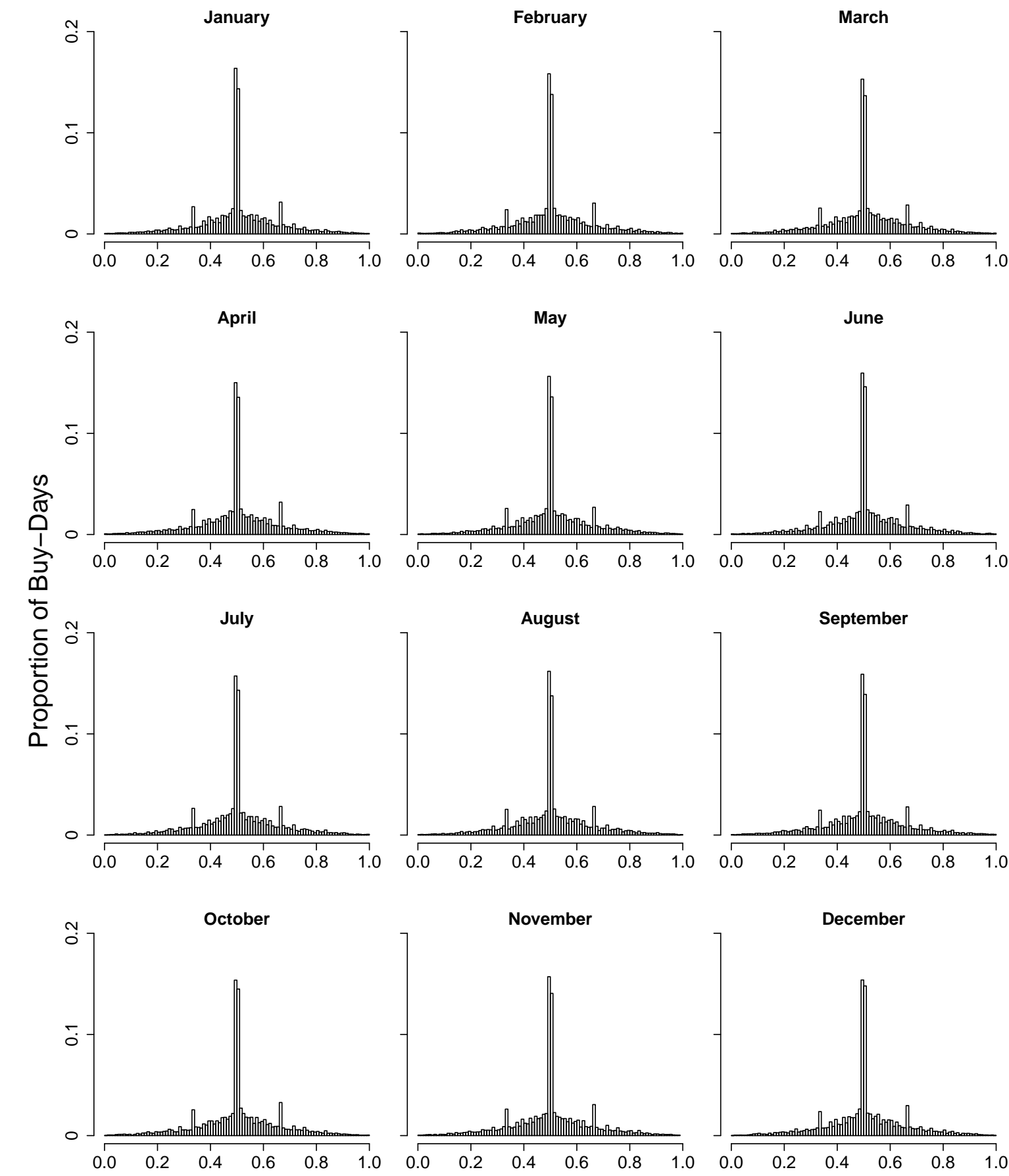

Proportion Invested in Stock A

Note: Figures show histograms of the proportion of the total buy-day investment (in pounds) which is invested in Stock A, where Stock A is a randomly chosen stock from the pair of stocks purchased. Sample comprises all buy-days on which investors buy exactly two stocks. Bin width is 0.01 . 
Figure A15: Proportion of Two-Stock Buy-Day Monies Invested in Stock A by Difference in Past 3 Month Stock Returns

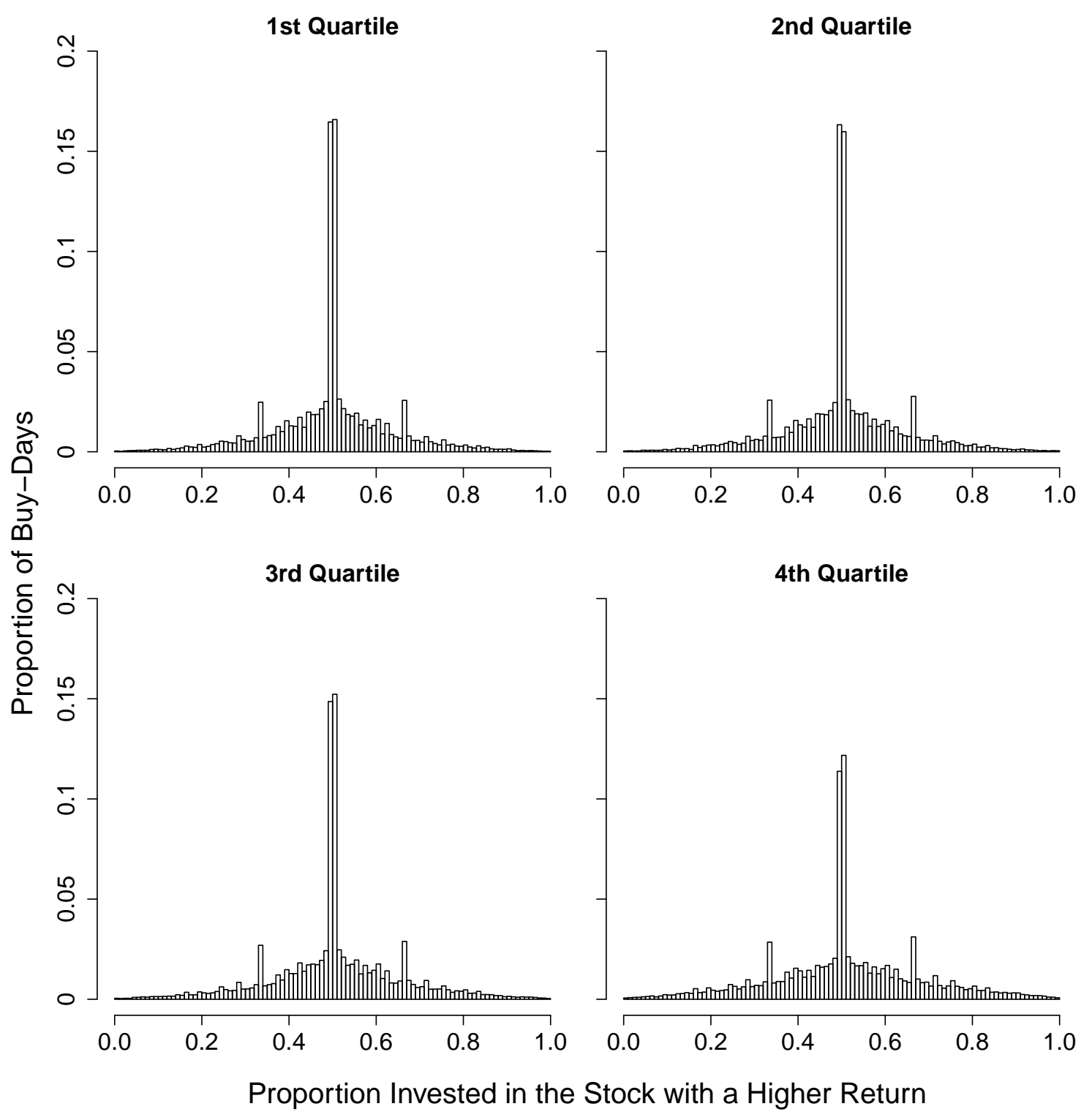

Note: Figures show histograms of the proportion of the total buy-day investment (in pounds) which is invested in Stock A, where Stock A is the stock from the pair of stocks purchased which has the higher gross returns over the previous 3 month period. Sample comprises all buy-days on which investors buy exactly two stocks. Bin width is 0.01 . 
Figure A16: Proportion of Two-Stock Buy-Day Monies Invested in Stock A by Difference in Next 3 Month Stock Returns

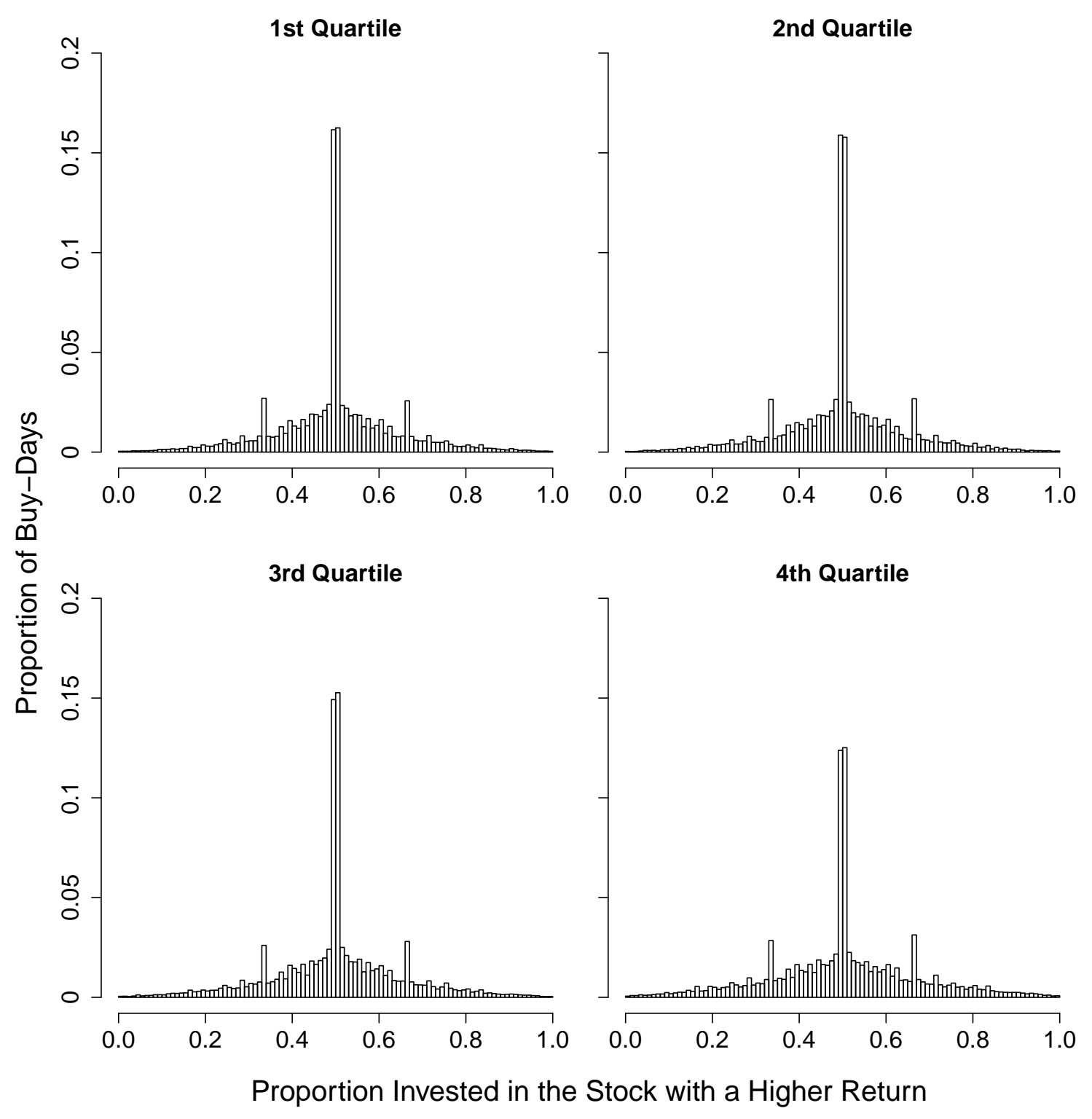

Note: Figures show histograms of the proportion of the total buy-day investment (in pounds) which is invested in Stock A, where Stock A is the stock from the pair of stocks purchased which has the higher gross returns over the next 3 month period. Sample comprises all buy-days on which investors buy exactly two stocks. Bin width is 0.01 . 
Figure A17: Naïve Buying Diversification by Two-Stock Buy-Days With Same Industry Stock Purchases and Different Industry Stock Purchases

(A) Proportion NBD

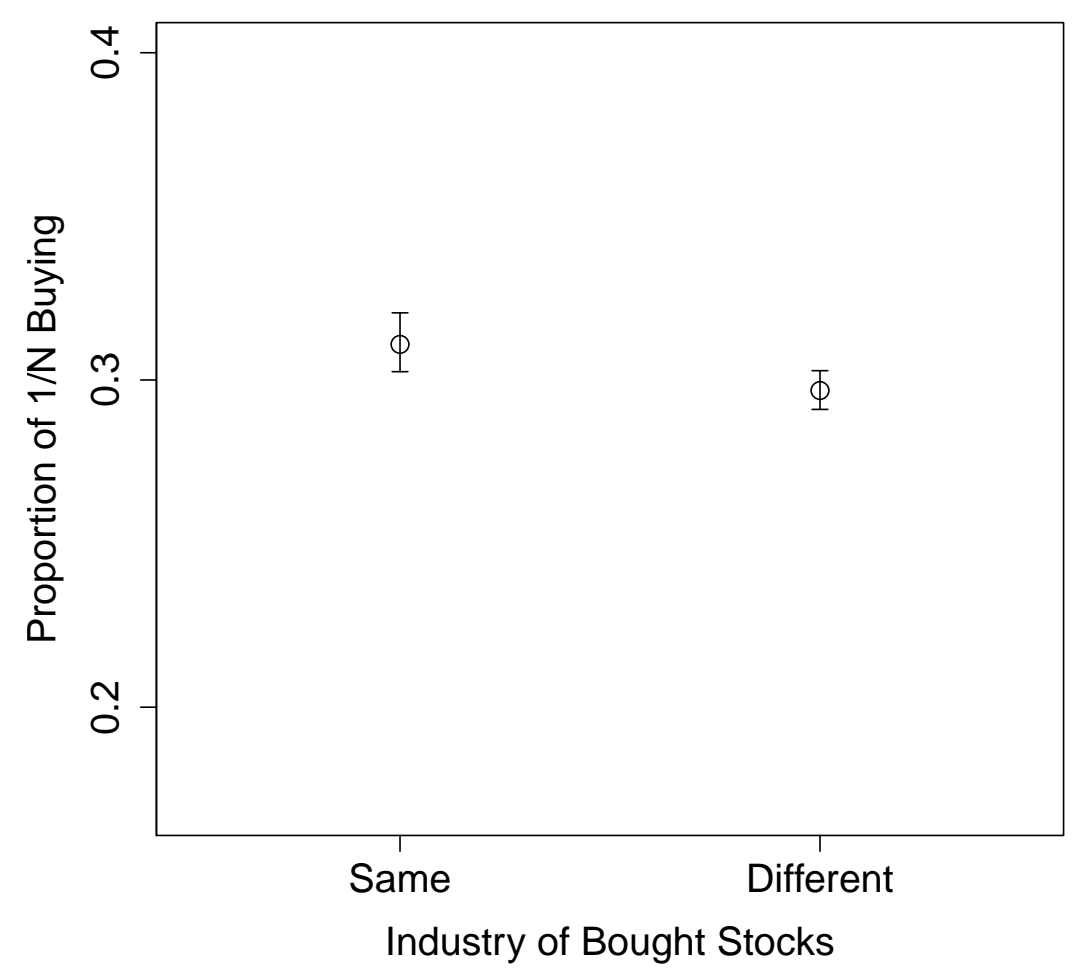

(B) Proportion of Two-Stock Buy-Day Investment in Stock A

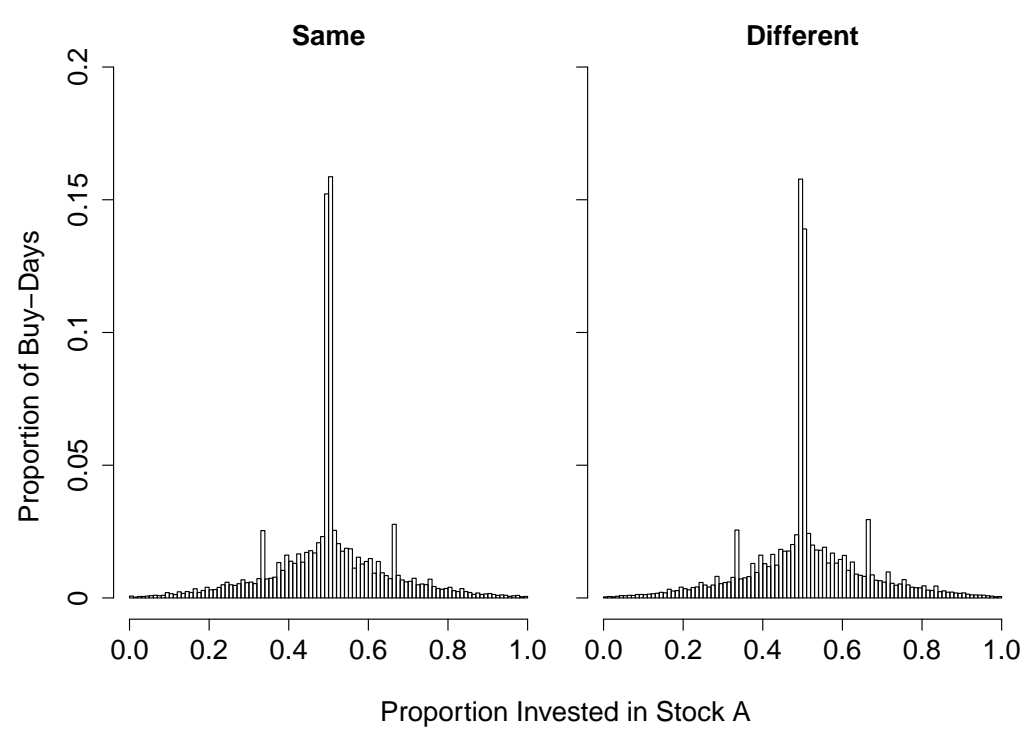

Note: Panel a shows proportion of all two-stock buy-days on which the buy-day investments are split equally (in pounds) across the two stocks by whether the two stocks are in the same, or different, industries. Panel B shows histograms of the proportion of the total buy-day investment (in pounds) which is invested in Stock A, where Stock A is a randomly chosen stock from the pair of stocks bought on the day. Sample comprises all buy-days on which investors buy exactly two stocks. Bin width is 0.01 . 
Figure A18: Proportion of Two-Stock Buy-Day Investment in Stock A Among New Accounts

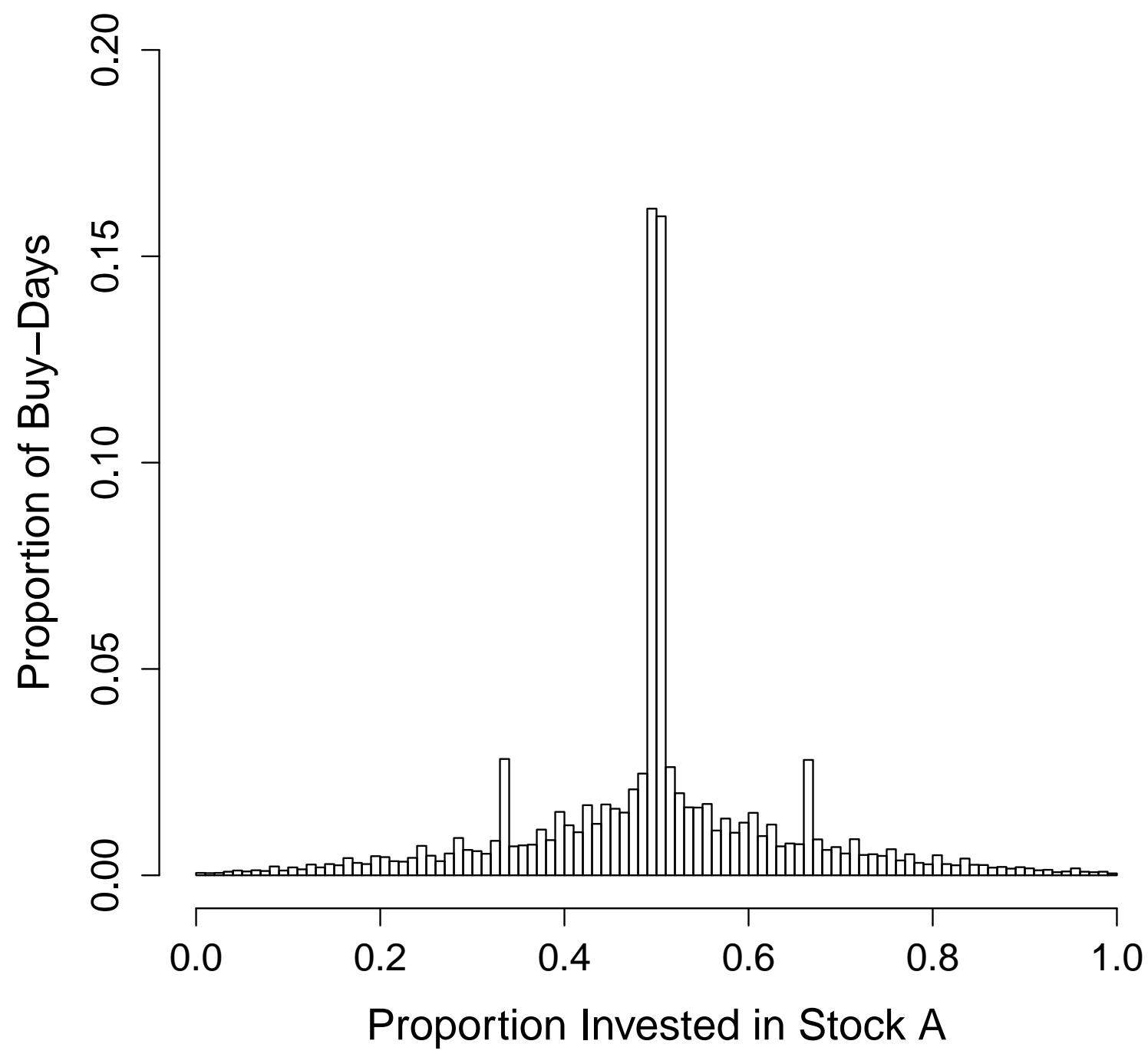

Note: Figures show histograms of the proportion of the total buy-day investment (in pounds) which is invested in Stock A, where Stock A is a randomly chosen stock from the pair of stocks purchased. Sample comprises all buy-days on which investors buy exactly two stocks in the new accounts data. Bin width is 0.01 . 
Figure A19: Proportion of Two-Stock Buy-Day Monies Invested in Stock A by Portfolio Value, New Accounts Only

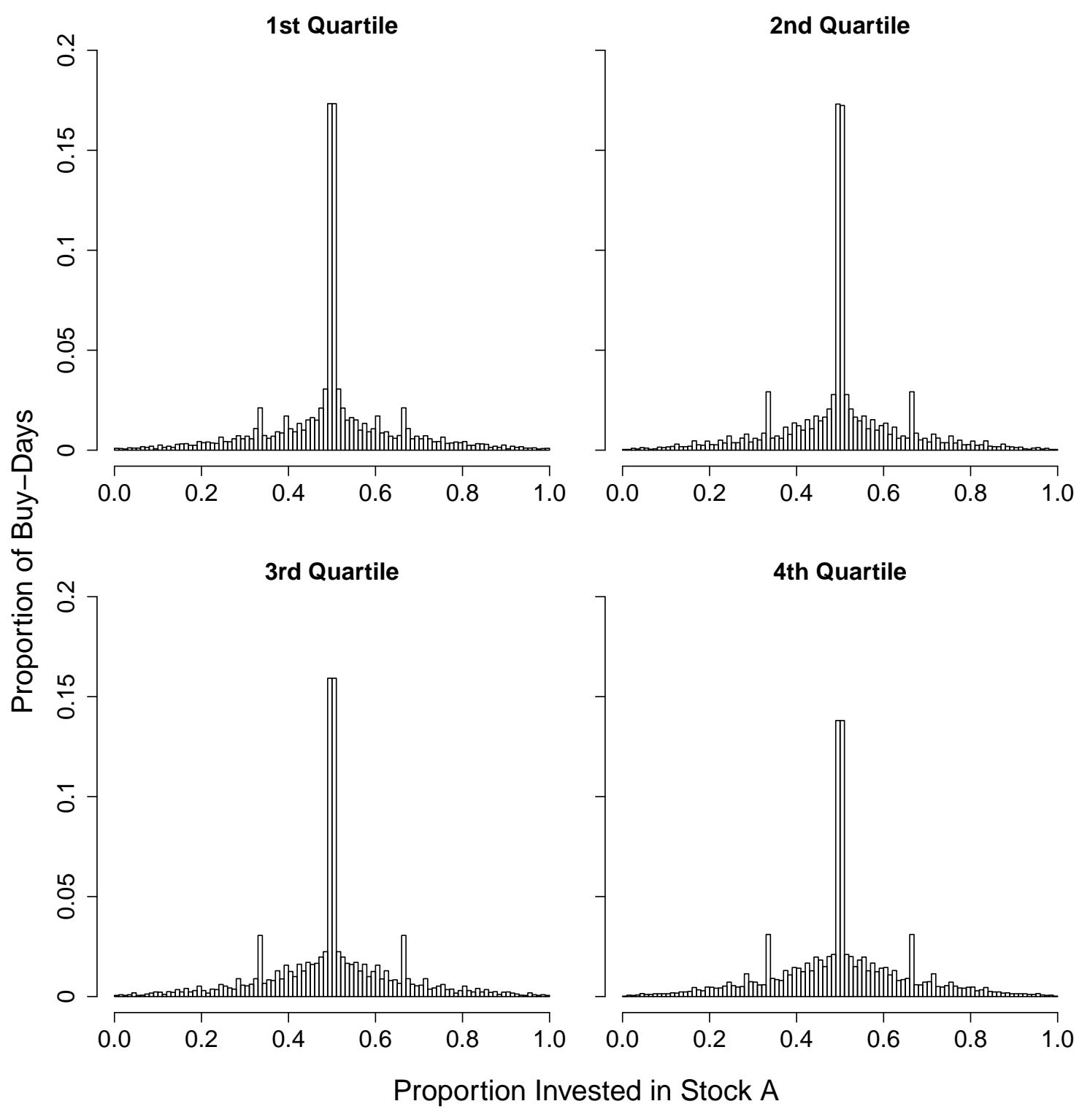

Note: Figures show histograms of the proportion of the total buy-day investment (in pounds) which is invested in Stock A, where Stock A is a randomly chosen stock from the pair of stocks purchased. Sample comprises all buy-days on which investors buy exactly two stocks in the new accounts data. Bin width is 0.01 . 
Figure A20: Proportion of Two-Stock Buy-Day Monies Invested in Stock A by Investment Amount on the Day

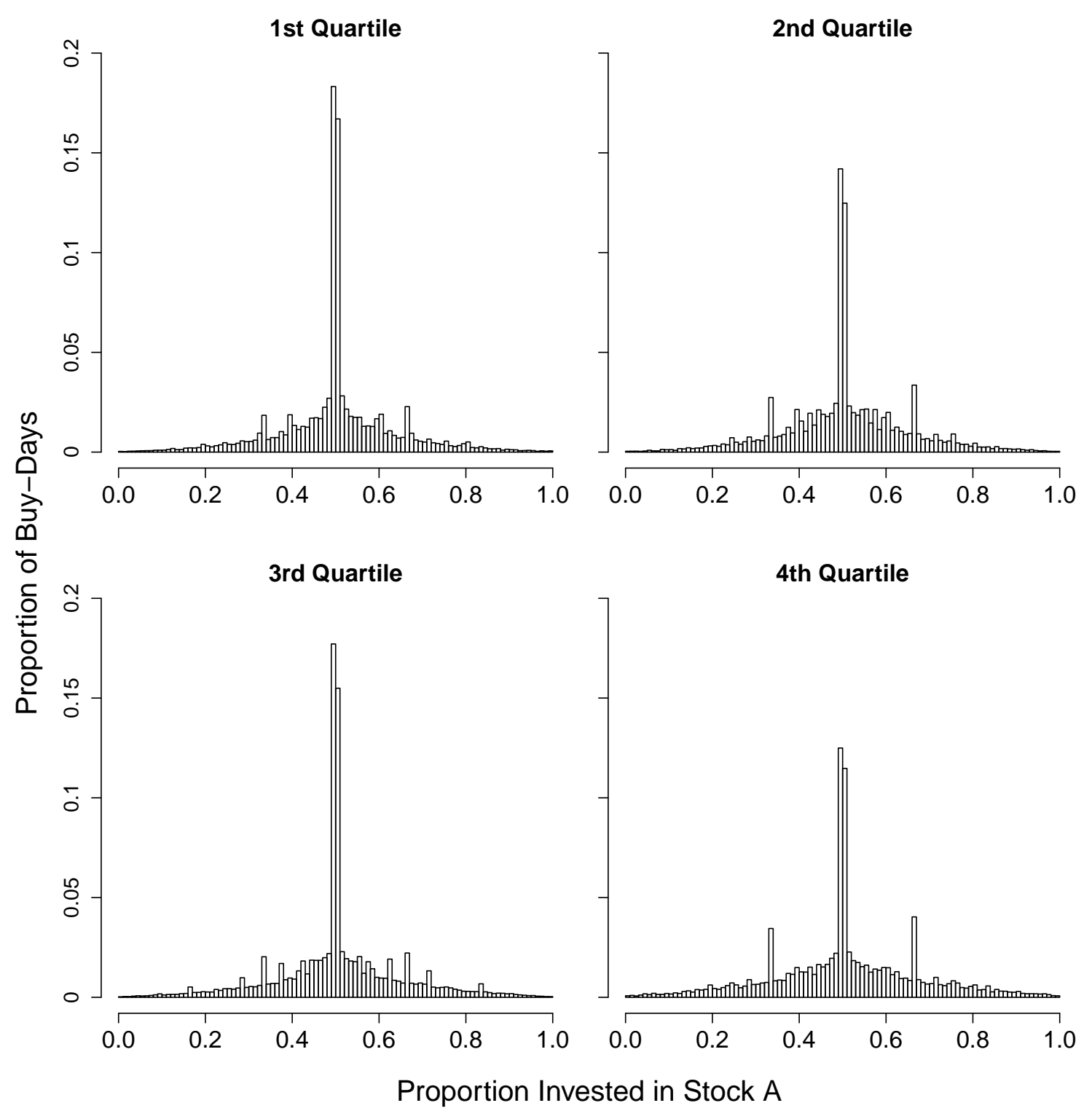

Note: Figures show histograms of the proportion of the total buy-day investment (in pounds) which is invested in Stock A, where Stock A is a randomly chosen stock from the pair of stocks purchased. Sample comprises all buy-days on which investors buy exactly two stocks. Bin width is 0.01 . 
Figure A21: Proportion of Two-Stock Buy-Day Monies Invested in Stock A by Number of Stocks in Portfolio, New Accounts Only

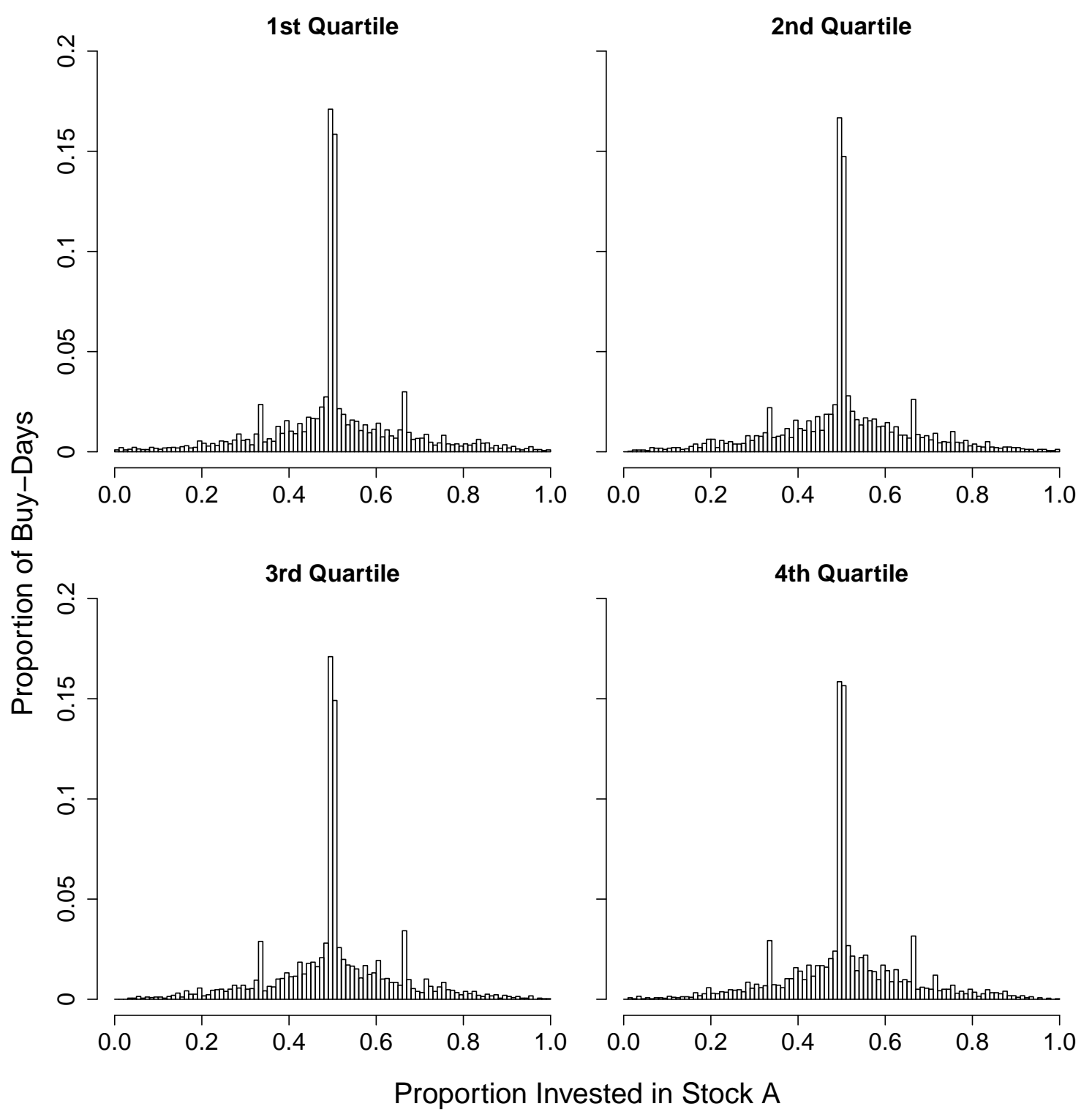

Note: Figures show histograms of the proportion of the total buy-day investment (in pounds) which is invested in Stock A, where Stock A is a randomly chosen stock from the pair of stocks purchased. Sample comprises all buy-days on which investors buy exactly two stocks in the new accounts data. Bin width is 0.01 . 
Figure A22: Proportion of Sell-Day Monies From Sale of Random Stock A, All Two-Stock Sell-Days

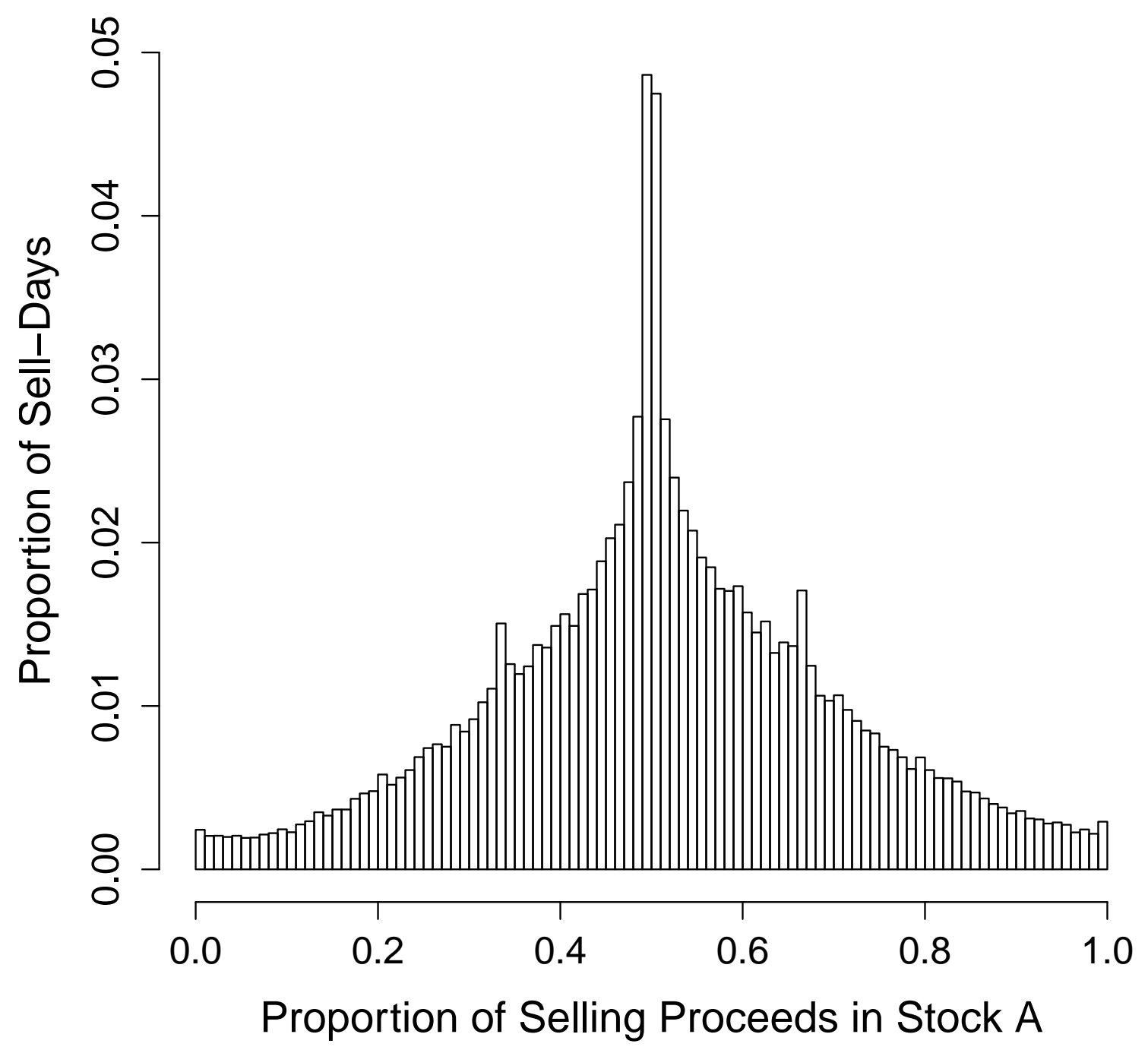

Note: Figures show histograms of the proportion of the total sell-day dis-investment (in pounds) which is sold out of Stock A, where Stock A is a randomly chosen stock from the pair of stocks sold. Bin width is 0.01 . 
Figure A23: Distribution of Total Buy-Day Investment (in $£$ ) Divided by Number of Stocks Bought on the Buy-Day

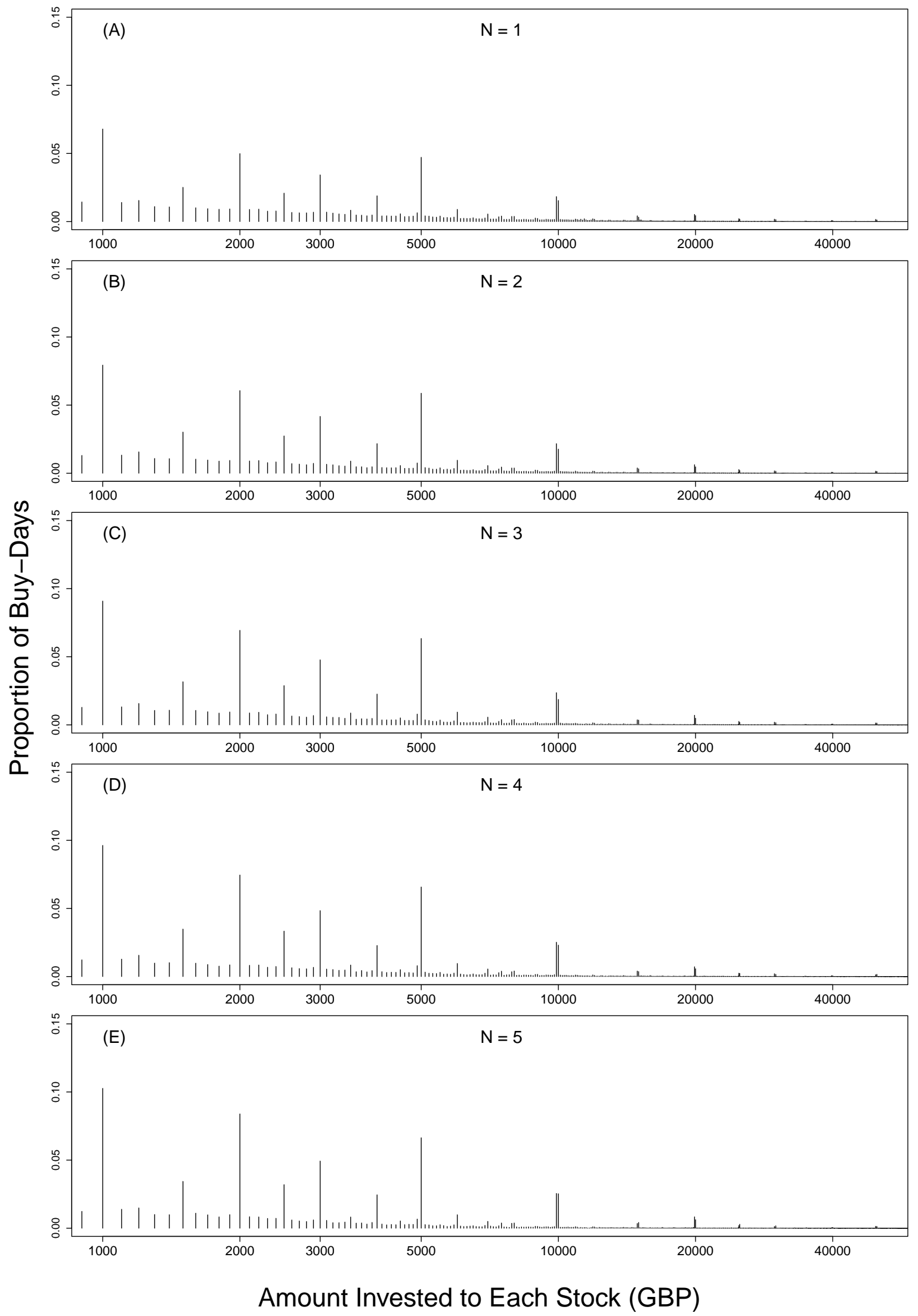

Note: Panels illustrate the distribution (density) of funds invested on the buy-day (in pounds) divided by the number of stocks purchased for all buy-days involving 1-5 stocks. 
Figure A24: Total Buy-Day Investment Amounts By Number Of Stocks Bought (A) Median Buy-Day Allocation

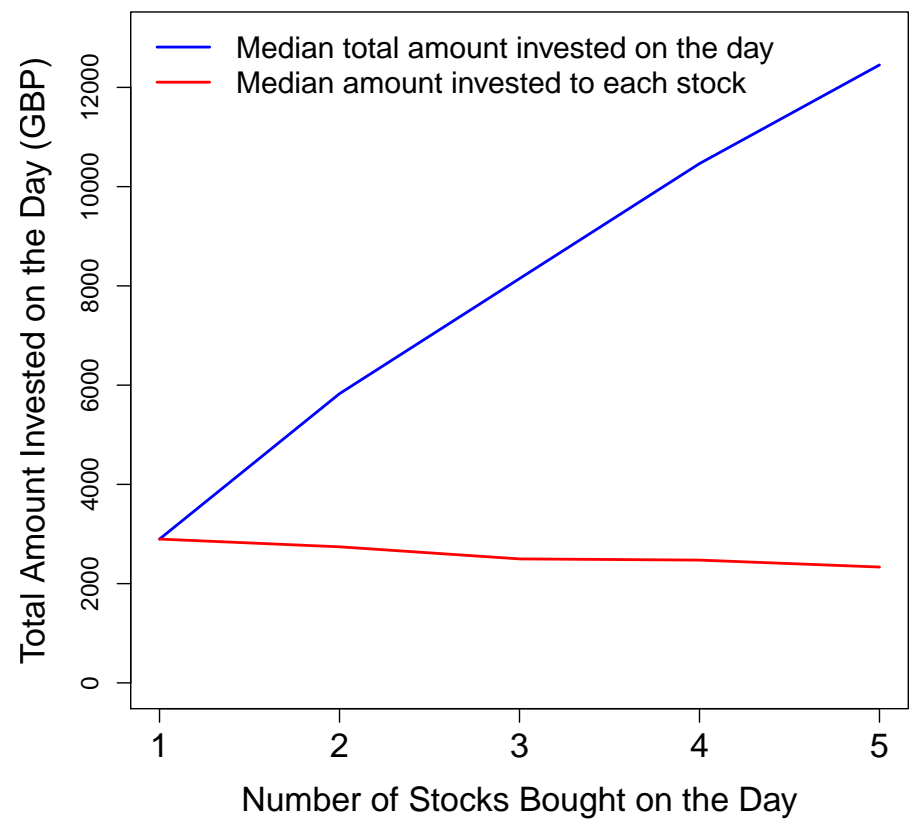

(B) Mean End of Day Portfolio Allocation

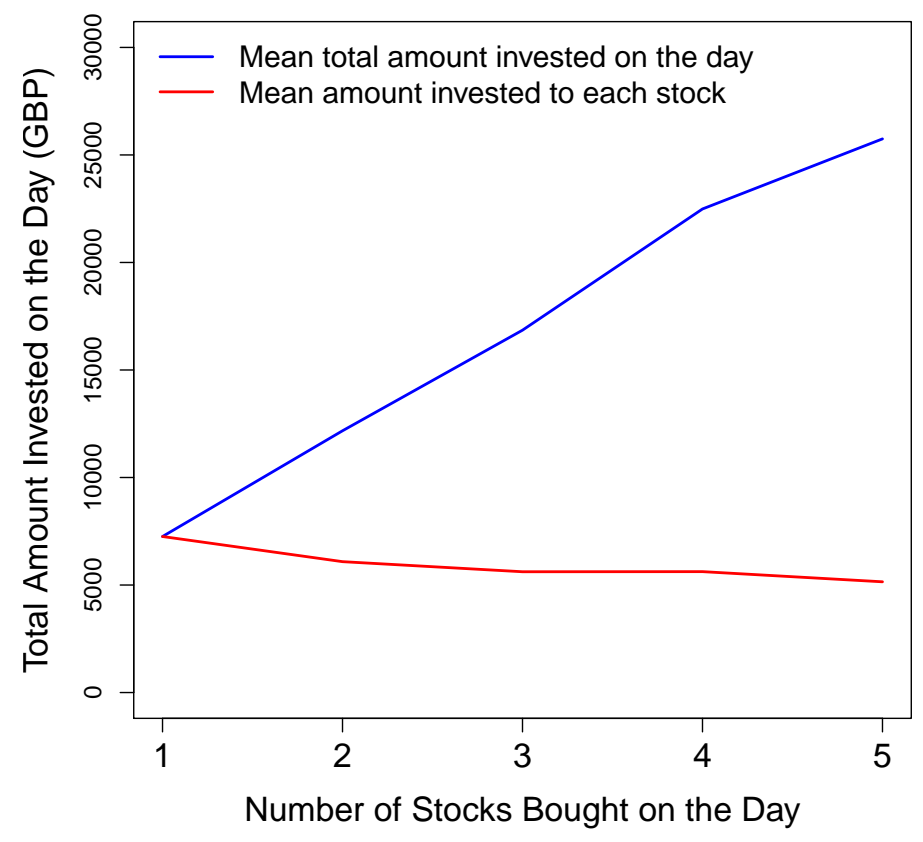

Note: Panel A figure shows line plots of the median total amount invested on the day and the median amount invested to each stock by number of stocks bought on the day. Panel B figure shows line plots for the mean total amount invested on the day and the mean amount invested to each stock by number of stocks bought on the day. 
Table A1: Summary Statistics for All Multiple-Stock Buy-Days

Panel (A)

\begin{tabular}{lccccc}
\hline & Mean & SD & 25th percentile & Median & 75th percentile \\
\hline Account Tenure & 49.06 & 45.66 & 11.07 & 34.97 & 77.30 \\
Investment Amount on the Day & $16,480.36$ & $50,154.36$ & $2,995.49$ & $7,005.23$ & $15,040.20$ \\
Range of Past 60-days Returns & 0.27 & 1.04 & 0.07 & 0.15 & 0.29 \\
Range of Next 60-days Returns & 0.22 & 0.25 & 0.07 & 0.15 & 0.28 \\
\hline
\end{tabular}

Panel (B)

\begin{tabular}{cccc}
\hline & Male & Female & Unknown \\
\hline Number of Accounts & 37,421 & 9,805 & 5,640 \\
\hline
\end{tabular}

Panel (C)

\begin{tabular}{lccccc}
\hline & Mean & SD & 25th percentile & Median & 75th percentile \\
\hline Ave. Num. of Trades per Month & 1.82 & 5.50 & 0.36 & 0.74 & 1.60 \\
\hline
\end{tabular}

Note: Table reports summary statistics for sample of multiple-stock buy-days. Unit of data is a single buy-day. Account tenure is number of months since account opennig. Range of Past / Next 60-day returns is the difference (in percent) in simple gross returns between stocks purchased on the buy-day. 
Table A2: Summary Statistics for New Account Multiple-Stock Buy-Days

\begin{tabular}{lccccc} 
Panel (A) & \multicolumn{7}{c}{} \\
\hline Statistics & Mean & SD & 25th percentile & Median & 75th percentile \\
\hline Account Tenure & 11.16 & 11.02 & 1.77 & 7.77 & 17.80 \\
Investment Amount on the Day & $11,457.93$ & $28,872.36$ & $2,001.93$ & $4,998.35$ & $10,914.70$ \\
Portfolio Value & $60,567.92$ & $187,747.84$ & $6,577.45$ & $17,328.90$ & $46,971.01$ \\
Num. of Stocks in the Portfolio & 8.29 & 9.10 & 3.00 & 5.00 & 10.00 \\
Range of Past 60-days Returns & 0.32 & 1.57 & 0.07 & 0.16 & 0.31 \\
Range of Next 60-days Returns & 0.24 & 0.28 & 0.08 & 0.16 & 0.30 \\
\hline
\end{tabular}

Panel (B)

\begin{tabular}{|c|c|c|c|c|c|c|}
\hline & Male & Female & Unknowr & & & \\
\hline Number of Accounts & 7,483 & 1,474 & 25 & & & \\
\hline \multicolumn{7}{|l|}{ Panel (C) } \\
\hline & & Mean & $\mathrm{SD}$ & 25th percentile & Median & 75th percentile \\
\hline Ave. Num. of Trades & r Mor & 1.49 & 3.82 & 0.32 & 0.66 & 1.43 \\
\hline
\end{tabular}

Note: Table reports summary statistics for sample of multiple-stock buy-days among the new accounts sample only. Unit of data is a single buy-day. Account tenure is number of months since account opening. Range of Past / Next 60-day returns is the difference (in percent) in simple gross returns between stocks purchased on the buy-day. 
Table A3: 1/N Allocations on ETF-and-Stock Buy-Days

(a) Panel A: $(£ P / N \times(1 \pm 0.02))$

\begin{tabular}{ccccc}
\hline$N$ & $1 / N$ Buying $(\%)$ & LL & UL & Buy-days \\
\hline 2 & 23.0 & 21.4 & 24.6 & 3116 \\
3 & 13.2 & 11.2 & 15.3 & 1459 \\
4 & 10.9 & 8.5 & 13.6 & 663 \\
5 & 14.1 & 10.5 & 17.9 & 355 \\
$6+$ & 6.9 & 4.5 & 9.8 & 562 \\
All & 17.4 & 16.2 & 18.6 & 6155 \\
\hline
\end{tabular}

(b) Panel B: $(£ P / N \times(1 \pm 0.05))$

\begin{tabular}{ccccc}
\hline$N$ & $1 / N$ Buying (\%) & LL & UL & Buy-days \\
\hline 2 & 29.1 & 27.4 & 30.9 & 3116 \\
3 & 14.9 & 12.8 & 17.2 & 1459 \\
4 & 13.0 & 10.4 & 15.7 & 663 \\
5 & 15.2 & 11.6 & 19.1 & 355 \\
$6+$ & 8.7 & 5.7 & 12.2 & 562 \\
All & 21.3 & 20.0 & 22.7 & 6155 \\
\hline
\end{tabular}

(c) Panel C: $(£ P / N \times(1 \pm 0.1))$

\begin{tabular}{ccccc}
\hline$N$ & $1 / N$ Buying (\%) & LL & UL & Buy-days \\
\hline 2 & 36.3 & 34.4 & 38.3 & 3116 \\
3 & 18.3 & 16.1 & 20.6 & 1459 \\
4 & 14.8 & 12.0 & 17.7 & 663 \\
5 & 17.2 & 13.4 & 21.4 & 355 \\
$6+$ & 10.1 & 6.5 & 14.1 & 562 \\
All & 26.2 & 24.8 & 27.7 & 6155 \\
\hline
\end{tabular}

Note: Table shows summary data for buy-days on which the investor bought at least one ETF together with at least one common stock. Each row reports the percentage of buy-days involving $N$ securities in which the proportion invested in each security falls within the $1 / N$ range, for differing ranges. See Section 3 for details. Lower limit and upper limit values of $95 \%$ confidence intervals from bootstrap mean estimates are reported in LL and UL columns. Sample is restricted to ETF-and-Stock buy-days. See Section 2 for details on the sample construction. 
Table A4: $1 / N$ Allocations on FTSE-100 ETF-and-Stock Buy-Days

(a) Panel A: $(£ P / N \times(1 \pm 0.02))$

\begin{tabular}{ccccc}
\hline$N$ & $1 / N$ Buying (\%) & LL & UL & Buy-days \\
\hline 2 & 21.7 & 18.4 & 25.5 & 635 \\
3 & 12.2 & 8.5 & 16.4 & 319 \\
4 & 8.4 & 4.5 & 12.8 & 154 \\
5 & 10.8 & 3.9 & 19.0 & 65 \\
$6+$ & 4.7 & 1.0 & 9.3 & 107 \\
All & 15.8 & 13.6 & 18.2 & 1280 \\
\hline
\end{tabular}

(b) Panel B: $(£ P / N \times(1 \pm 0.05))$

\begin{tabular}{ccccc}
\hline$N$ & $1 / N$ Buying $(\%)$ & LL & UL & Buy-days \\
\hline 2 & 26.8 & 23.1 & 30.9 & 635 \\
3 & 12.5 & 8.9 & 16.9 & 319 \\
4 & 10.4 & 5.2 & 15.4 & 154 \\
5 & 12.3 & 5.0 & 21.9 & 65 \\
$6+$ & 4.7 & 0.9 & 9.5 & 107 \\
All & 18.7 & 16.2 & 21.3 & 1280 \\
\hline
\end{tabular}

(c) Panel C: $(£ P / N \times(1 \pm 0.10))$

\begin{tabular}{ccccc}
\hline$N$ & $1 / N$ Buying (\%) & LL & UL & Buy-days \\
\hline 2 & 33.2 & 29.5 & 37.5 & 635 \\
3 & 15.0 & 11.1 & 19.8 & 319 \\
4 & 11.7 & 6.8 & 17.4 & 154 \\
5 & 16.9 & 8.5 & 27.4 & 65 \\
$6+$ & 5.6 & 1.8 & 10.7 & 107 \\
All & 23.0 & 20.6 & 25.6 & 1280 \\
\hline
\end{tabular}

Note: Table shows summary data for buy-days on which the investor bought at least one FTSE100 Index ETF together with at least one common stock. Each row reports the percentage of buy-days involving $N$ securities in which the proportion invested in each security falls within the $1 / N$ range, for differing ranges. See Section 3 for details. Lower limit and upper limit values of $95 \%$ confidence intervals from bootstrap mean estimates are reported in LL and UL columns. Sample is restricted to ETF-and-Stock buy-days. See Section 2 for details on the sample construction. 
Table A5: Naïve Buying Diversification by Investor Characteristics

(a) Gender

\begin{tabular}{lcc}
\hline Gender & Male & Female \\
\hline \% of Sample & 83.29 & 16.71 \\
Proportion of $1 / N$ & 0.29 & 0.30 \\
$95 \%$ CI & {$[0.28,0.29]$} & {$[0.29,0.32]$} \\
\hline
\end{tabular}

(b) Decade of Birth

\begin{tabular}{lcccc}
\hline Decade of Birth & $30 \mathrm{~s}$ & $40 \mathrm{~s}$ & $50 \mathrm{~s}$ & $60 \mathrm{~s}$ \\
\hline \% of Sample & 3.81 & 15.57 & 24.14 & 28.76 \\
Proportion 1/N & 0.31 & 0.28 & 0.29 & 0.30 \\
$95 \%$ CI & {$[0.28,0.34]$} & {$[0.27,0.30]$} & {$[0.27,0.30]$} & {$[0.29,0.31]$} \\
\hline
\end{tabular}

\begin{tabular}{lcccc} 
Decade of Birth & $70 \mathrm{~s}$ & $80 \mathrm{~s}$ & $90 \mathrm{~s}$ & $2000-$ \\
\hline \% of Sample & 16.32 & 8.63 & 2.17 & 0.14 \\
Proportion 1/N & 0.29 & 0.32 & 0.30 & 0.32 \\
$95 \%$ CI & {$[0.28,0.30]$} & {$[0.31,0.34]$} & {$[0.28,0.32]$} & {$[0.26,0.40]$} \\
\hline
\end{tabular}

(c) Account Age

\begin{tabular}{lcccc}
\hline & \multicolumn{4}{c}{ Quartiles } \\
Age in Months & Q1 & Q2 & Q3 & Q4 \\
\hline Quartile Mean & 9.94 & 41.84 & 83.67 & 134.26 \\
Proportion 1/N & 0.31 & 0.29 & 0.28 & 0.27 \\
$95 \%$ CI & {$[0.30,0.32]$} & {$[0.28,0.30]$} & {$[0.27,0.29]$} & {$[0.25,0.28]$} \\
\hline
\end{tabular}

(d) Trading Frequency

\begin{tabular}{lcccc}
\hline & \multicolumn{4}{c}{ Quartiles } \\
Trades Per Month & Q1 & Q2 & Q3 & Q4 \\
\hline Quartile Mean & 0.56 & 1.93 & 5.78 & 35.07 \\
Proportion 1/N & 0.36 & 0.33 & 0.27 & 0.19 \\
$95 \%$ CI & {$[0.35,0.36]$} & {$[0.32,0.34]$} & {$[0.26,0.29]$} & {$[0.17,0.21]$} \\
\hline
\end{tabular}

Note: Table shows summary data the proportion of two-stock buy-days within the $1 / N$ range. See Section 3 for details. Sample is restricted to multiple-stock buy-days. See Section 2 for details on the sample construction. 
Table A6: Naïve Buying Diversification by Trading Characteristics I

(a) Day of the Week

\begin{tabular}{lccccc}
\hline Day of the Week & Mon & Tue & Wed & Thu & Fri \\
\hline \% of Sample & 20.76 & 20.35 & 19.40 & 19.45 & 20.04 \\
Proportion 1/N & 0.31 & 0.29 & 0.29 & 0.29 & 0.29 \\
$95 \%$ CI & {$[0.31,0.32]$} & {$[0.29,0.30]$} & {$[0.29,0.30]$} & {$[0.28,0.30]$} & {$[0.29,0.30]$} \\
\hline
\end{tabular}

(b) Month of the Year

\begin{tabular}{lcccccc}
\hline Month & Jan & Feb & Mar & Apr & May & Jun \\
\hline \% of Sample & 9.48 & 8.70 & 9.57 & 10.58 & 9.68 & 7.61 \\
Proportion 1/N & 0.31 & 0.30 & 0.29 & 0.29 & 0.29 & 0.31 \\
$95 \%$ CI & {$[0.30,0.32]$} & {$[0.29,0.31]$} & {$[0.28,0.30]$} & {$[0.28,0.29]$} & {$[0.28,0.30]$} & {$[0.30,0.32]$} \\
\hline & & & & & & \\
Month & Jul & Aug & Sep & Oct & Nov & Dec \\
\hline \% of Sample & 7.53 & 7.53 & 7.62 & 8.14 & 7.40 & 6.16 \\
Proportion 1/N & 0.30 & 0.30 & 0.30 & 0.30 & 0.30 & 0.30 \\
95\% CI & {$[0.29,0.31]$} & {$[0.29,0.31]$} & {$[0.29,0.31]$} & {$[0.29,0.31]$} & {$[0.29,0.31]$} & {$[0.29,0.31]$} \\
\hline
\end{tabular}

(c) Difference in Past 3 Month Gross Returns

\begin{tabular}{lcccc}
\hline & \multicolumn{4}{c}{ Quartile } \\
Difference in Past 3 Month Returns (\%) & Q1 & Q2 & Q3 & Q4 \\
\hline Quartile Mean & 0.03 & 0.09 & 0.18 & 0.83 \\
Proportion 1/N & 0.33 & 0.32 & 0.30 & 0.24 \\
$95 \%$ CI & {$[0.32,0.34]$} & {$[0.32,0.33]$} & {$[0.29,0.31]$} & {$[0.23,0.24]$} \\
\hline
\end{tabular}

(d) Difference in Next 3 Month Gross Returns

\begin{tabular}{lcccc}
\hline & \multicolumn{4}{c}{ Quartile } \\
Difference in Next 3 Month Returns (\%) & Q1 & Q2 & Q3 & Q4 \\
\hline Quartile Mean & 0.03 & 0.09 & 0.17 & 0.48 \\
Proportion 1/N & 0.32 & 0.32 & 0.30 & 0.25 \\
$95 \%$ CI & {$[0.32,0.33]$} & {$[0.31,0.32]$} & {$[0.29,0.31]$} & {$[0.24,0.26]$} \\
\hline
\end{tabular}

Note: Table shows summary data the proportion of two-stock buy-days within the $1 / N$ range. See Section 3 for details. Sample is restricted to multiple-stock buy-days. See Section 2 for details on the sample construction. 
Table A7: Naïve Buying Diversification by Trading Characteristics II

(a) Portfolio Value $(£)$

\begin{tabular}{lcccc}
\hline & \multicolumn{4}{c}{ Quartile } \\
Portfolio Value $(£)$ & Q1 & Q2 & Q3 & Q4 \\
\hline Quartile Mean & $2,566.81$ & $10,326.33$ & $25,908.63$ & $168,976.48$ \\
Proportion 1/N & 0.35 & 0.35 & 0.32 & 0.28 \\
$95 \%$ CI & {$[0.33,0.36]$} & {$[0.33,0.36]$} & {$[0.30,0.34]$} & {$[0.25,0.30]$} \\
\hline
\end{tabular}

(b) Investment Value $(£)$

\begin{tabular}{lcccc}
\hline & \multicolumn{4}{c}{ Quartile } \\
Investment Value $(£)$ & $\mathrm{Q} 1$ & $\mathrm{Q} 2$ & $\mathrm{Q} 3$ & $\mathrm{Q} 4$ \\
\hline Quartile Mean & $1,441.06$ & $4,035.69$ & $8,489.37$ & $34,723.43$ \\
Proportion 1/N & 0.35 & 0.27 & 0.33 & 0.24 \\
$95 \%$ CI & {$[0.34,0.36]$} & {$[0.26,0.28]$} & {$[0.32,0.34]$} & {$[0.23,0.25]$} \\
\hline
\end{tabular}

(c) Holds Existing Position(s)

\begin{tabular}{lcc}
\hline Existing Position Held & With & Without \\
\hline \% of Sample & 82.76 & 17.24 \\
Proportion $1 / N$ & 0.31 & 0.39 \\
$95 \%$ CI & {$[0.29,0.32]$} & {$[0.38,0.41]$} \\
\hline
\end{tabular}

(d) Number of Stocks Held

\begin{tabular}{lcccc}
\hline & \multicolumn{4}{c}{ Quartile } \\
Number of Stocks Held & Q1 & Q2 & Q3 & Q4 \\
\hline Quartile Mean & 2.35 & 4.44 & 7.22 & 17.90 \\
Proportion $1 / N$ & 0.33 & 0.31 & 0.32 & 0.32 \\
$95 \%$ CI & {$[0.32,0.34]$} & {$[0.29,0.33]$} & {$[0.30,0.34]$} & {$[0.29,0.35]$} \\
\hline
\end{tabular}

(e) Same Day Sale

\begin{tabular}{lcc}
\hline Sale on the Day & With & Without \\
\hline \% of Sample & 20.22 & 79.78 \\
Proportion 1/N & 0.22 & 0.35 \\
$95 \%$ CI & {$[0.19,0.24]$} & {$[0.34,0.36]$} \\
\hline
\end{tabular}

Note: Table shows summary data the proportion of two-stock buy-days within the $1 / N$ range. See Section 3 for details. Sample is restricted to multiple-stock buy-days. See Section 2 for details on the sample construction. 
Table A8: Proportion of Sell-Days on Which Investor Splits Sales $1 / N$

(a) Panel A: $(£ P / N \times(1 \pm 0.02))$

\begin{tabular}{ccccc}
\hline$N$ (Num. Sold Stocks) & $1 / N$ Selling $(\%)$ & LL & UL & Sell-Days \\
\hline 2 & 9.6 & 9.2 & 10.0 & 106971 \\
3 & 1.6 & 1.4 & 1.9 & 38413 \\
4 & 0.8 & 0.6 & 1.0 & 15493 \\
5 & 0.5 & 0.3 & 0.7 & 7306 \\
$6+$ & 0.1 & 0.0 & 0.2 & 11578 \\
All & 6.2 & 5.9 & 6.4 & 179761 \\
\hline
\end{tabular}

Note: Table shows summary data for multiple-stock sell-days. Each row reports the percentage of sell-days involving $N$ stocks in which the proportion invested in each stock falls within the $1 / N$ range, for differing ranges. Lower limit and upper limit values of $95 \%$ confidence intervals from bootstrap mean estimate reported in LL and UL columns. 
Table A9: Proportion of Sell-Days on Which Investors Achieve $1 / N$ Portfolios

\begin{tabular}{|c|c|c|c|c|}
\hline $\begin{array}{l}\text { Existing } \\
\text { Positions }\end{array}$ & $\begin{array}{c}\text { Sold } \\
\text { Stocks }\end{array}$ & $\begin{array}{l}\text { Resulting } \\
\text { Positions }\end{array}$ & $\begin{array}{l}1 / N \text { Resulting } \\
\text { Portfolios }(\%)\end{array}$ & $\begin{array}{l}\text { Num. } \\
\text { Sell-Days }\end{array}$ \\
\hline 2 & 1 & 2 & 2.91 & 2746 \\
\hline 2 & 2 & 2 & 4.90 & 102 \\
\hline 3 & 1 & 2 & 5.36 & 4947 \\
\hline 3 & 1 & 3 & 0.19 & 1563 \\
\hline 3 & 2 & 2 & 2.82 & 142 \\
\hline 3 & 2 & 3 & 0.00 & 75 \\
\hline 3 & 3 & 2 & 14.29 & 14 \\
\hline 3 & 3 & 3 & 0.00 & 14 \\
\hline 4 & 1 & 3 & 0.63 & 3644 \\
\hline 4 & 1 & 4 & 0.00 & 1106 \\
\hline 4 & 2 & 2 & 9.73 & 442 \\
\hline 4 & 2 & 3 & 0.00 & 105 \\
\hline 4 & 2 & 4 & 0.00 & 86 \\
\hline 4 & 3 & 2 & 5.56 & 18 \\
\hline 4 & 3 & 3 & 0.00 & 16 \\
\hline 4 & 3 & 4 & 0.00 & 10 \\
\hline 4 & 4 & 2 & 0.00 & 3 \\
\hline 4 & 4 & 3 & 0.00 & 5 \\
\hline 4 & 4 & 4 & 0.00 & 12 \\
\hline 5 & 1 & 4 & 0.11 & 2631 \\
\hline 5 & 1 & 5 & 0.00 & 855 \\
\hline 5 & 2 & 3 & 1.59 & 314 \\
\hline 5 & 2 & 4 & 0.00 & 68 \\
\hline 5 & 2 & 5 & 0.00 & 56 \\
\hline 5 & 3 & 2 & 4.44 & 90 \\
\hline 5 & 3 & 3 & 0.00 & 15 \\
\hline 5 & 3 & 4 & 0.00 & 5 \\
\hline 5 & 3 & 5 & 0.00 & 14 \\
\hline 5 & 4 & 2 & 0.00 & 4 \\
\hline 5 & 4 & 3 & 0.00 & 2 \\
\hline 5 & 4 & 4 & 0.00 & 1 \\
\hline 5 & 4 & 5 & 0.00 & 1 \\
\hline 5 & 5 & 2 & 0.00 & 2 \\
\hline $6+$ & 1 & 5 & 0.05 & 2089 \\
\hline $6+$ & 1 & $6+$ & 0.02 & 12126 \\
\hline $6+$ & 2 & 4 & 1.13 & 265 \\
\hline $6+$ & 2 & 5 & 0.00 & 271 \\
\hline $6+$ & 2 & $6+$ & 0.00 & 1689 \\
\hline $6+$ & 3 & 3 & 1.39 & 72 \\
\hline $6+$ & 3 & 4 & 0.00 & 64 \\
\hline $6+$ & 3 & 5 & 0.00 & 62 \\
\hline $6+$ & 3 & $6+$ & 0.00 & 493 \\
\hline $6+$ & 4 & 2 & 6.67 & 30 \\
\hline $6+$ & 4 & 3 & 0.00 & 29 \\
\hline $6+$ & 4 & 4 & 0.00 & 31 \\
\hline $6+$ & 4 & 5 & 3.70 & 27 \\
\hline $6+$ & 4 & $6+$ & 0.00 & 198 \\
\hline $6+$ & 5 & 2 & 5.88 & 17 \\
\hline $6+$ & 5 & 3 & 0.00 & 13 \\
\hline $6+$ & 5 & 4 & 0.00 & 14 \\
\hline $6+$ & 5 & 5 & 0.00 & 12 \\
\hline $6+$ & 5 & $6+$ & 0.00 & 110 \\
\hline $6+$ & $6+$ & 2 & 8.00 & 25 \\
\hline $6+$ & $6+$ & 3 & 6.67 & 15 \\
\hline $6+$ & $6+$ & 4 & 0.00 & 12 \\
\hline $6+$ & $6+$ & 581 & 0.00 & 18 \\
\hline $6+$ & $6+$ & $6+$ & 0.00 & 117 \\
\hline
\end{tabular}

Note: Table shows summary data for multiple-stock sell-days. 
Table A10: Proportion of Sell-Days with NPD by Number of Stocks, Feasible NPD Sample

\begin{tabular}{lcccc}
\hline$N$ & $1 / N$ Portfolios $(\%)$ & LL & UL & Buy-days \\
\hline 2 & 10.2 & 9.1 & 11.2 & 3004 \\
3 & 2.6 & 1.5 & 3.8 & 1006 \\
4 & 1.4 & 0.3 & 2.5 & 370 \\
5 & 1.1 & 0.0 & 2.8 & 183 \\
$6+$ & 1.1 & 0.0 & 2.8 & 176 \\
All & 7.2 & 6.5 & 7.9 & 4739 \\
\hline
\end{tabular}

Note: Table shows summary data for multiple-stock sell-days. Each row reports the percentage of selldays resulting positions in the $1 / N$ range, for different number of stocks in the portfolio. Lower limit and upper limit values of $95 \%$ confidence intervals from bootstrap mean estimate reported in LL and UL columns. Sample is restricted to multiple-stock sell-days in the new accounts data. See Section 2 for details on the sample construction. 
Table A11: Proportion of Buy-and-Sell Days with NPD by Number of Stocks, Feasible NPD Sample

\begin{tabular}{lcccc}
\hline$N$ & $1 / N$ Portfolio $(\%)$ & LL & UL & Buy-days \\
\hline 2 & 17.4 & 13.5 & 21.5 & 357 \\
3 & 3.0 & 1.6 & 4.5 & 498 \\
4 & 0.0 & 0.0 & 0.0 & 513 \\
5 & 0.5 & 0.0 & 1.3 & 400 \\
$6+$ & 0.0 & 0.0 & 0.1 & 3167 \\
All & 1.6 & 1.2 & 2.1 & 4935 \\
\hline
\end{tabular}

Note: Table shows summary data for days on which investors make both multiple-stock buy trades and sell trades. Each row reports the percentage of days resulting positions in the $1 / N$ range, for different number of stocks in the portfolio. Lower limit and upper limit values of $95 \%$ confidence intervals from bootstrap mean estimate reported in LL and UL columns. Sample is restricted to days on which investors make both multiple-stock buy trades and sell trade in the new accounts data. See Section 2 for details on the sample construction. 